\title{
Mitochondrial and Sarcoplasmic Reticulum Interconnection in Cardiac Arrhythmia
}

\author{
Felipe Salazar-Ramírez', Roberto Ramos-Mondragón ${ }^{2,3}$ and Gerardo García-Rivas 1,4,5* \\ ${ }^{1}$ Tecnologico de Monterrey, Escuela de Medicina y Ciencias de la Salud, Cátedra de Cardiología y Medicina Cardiovascular, \\ Monterrey, Mexico, '2 Department of Pharmacology, University of Michigan Medical School, Ann Arbor, MI, United States, \\ ${ }^{3}$ Department of Internal Medicine, Division of Cardiovascular Medicine, University of Michigan Medical School, Ann Arbor, \\ MI, United States, ${ }^{4}$ TecSalud, Centro de Investigación Biomédica, Hospital Zambrano-Hellion, San Pedro Garza García, \\ Mexico, ${ }^{5}$ TecSalud, Centro de Medicina Funcional, Hospital Zambrano-Hellion, San Pedro Garza García, Mexico
}

$\mathrm{Ca}^{2+}$ plays a pivotal role in mitochondrial energy production, contraction, and apoptosis. Mitochondrial $\mathrm{Ca}^{2+}$-targeted fluorescent probes have demonstrated that mitochondria $\mathrm{Ca}^{2+}$ transients are synchronized with $\mathrm{Ca}^{2+}$ fluxes occurring in the sarcoplasmic reticulum (SR). The presence of specialized proteins tethering SR to mitochondria ensures the local $\mathrm{Ca}^{2+}$ flux between these organelles. Furthermore, communication between SR and mitochondria impacts their functionality in a bidirectional manner. Mitochondrial $\mathrm{Ca}^{2+}$ uptake through the mitochondrial $\mathrm{Ca}^{2+}$ uniplex is essential for ATP production and controlled reactive oxygen species levels for proper cellular signaling. Conversely, mitochondrial ATP ensures the proper functioning of SR $\mathrm{Ca}^{2+}$-handling proteins, which ensures that mitochondria receive an adequate supply of $\mathrm{Ca}^{2+}$. Recent evidence suggests that altered $\mathrm{SR} \mathrm{Ca}^{2+}$ proteins, such as ryanodine receptors and the sarco/endoplasmic reticulum $\mathrm{Ca}^{2+}$ ATPase pump, play an important role in maintaining proper cardiac membrane excitability, which may be initiated and potentiated when mitochondria are dysfunctional. This recognized mitochondrial role offers the opportunity to develop new therapeutic approaches aimed at preventing cardiac arrhythmias in cardiac disease.

Keywords: mitochondria, sarcoplasmic reticulum, arrhythmia, calcium, heart failure, ischemia/reperfusion injury

\section{INTRODUCTION}

Arrhythmias can be defined as any disturbance in the normal electrical sequence of the heart. These disturbances may cause the electrical impulse to travel slowly, rapidly, or in an erratic manner. However, few studies have calculated the overall burden of arrhythmias. Incidence has been reported to be about $2.35 \%$ in the United Kingdom's general population (Khurshid et al., 2018). In Mexico, only data for atrial fibrillation (AF) exist, and it is estimated to affect $2 \%$ of the general population (Lara-Vaca et al., 2014). These data are consistent with the estimated global rates of approximately 1-4\% (Zulkifly et al., 2018). Although it may seem like a low percentage, electrical abnormalities appear in up to $39 \%$ of patients with cardiopathies (Vazquez Ruiz de Castroviejo et al., 2005). Within this population, sudden cardiac death constitutes a significant cause of mortality. Sudden cardiac death is defined as when the death of a patient occurs, most commonly by a fatal ventricular arrhythmia, within $1 \mathrm{~h}$ of the onset of symptoms when there is a witness or within $24 \mathrm{~h}$ of last 
being seen alive when no witness is available (Adabag et al., 2010). Arrhythmia susceptibility is especially concerning in high-risk populations, such as heart failure (HF) patients, in which the incidence of sudden cardiac death reaches approximately $15 \%$ per year; this population continues to grow, as it is the outcome of almost all cardiovascular pathologies (Lee et al., 2011; Srinivasan and Schilling, 2018). Sudden cardiac death has been documented to be responsible for $20-30 \%$ of all cardiac deaths worldwide and about 7-18\% in the United States (Rodríguez-Reyes et al., 2015). Analyzing the process of arrhythmogenesis at the cellular level is, therefore, of vital importance to better understand the underlying mechanisms that lead to its development and elucidate new potential therapeutic targets to prevent it.

Focal activity and re-entry are proposed as the main mechanisms of cardiac arrhythmia. Re-entry is associated with conduction abnormalities and occurs when a propagating impulse fails to extinguish after normal activation of the heart tissue and re-excites the heart after completion of the refractory period. A reduction in the cardiac impulse's wavelength, which is a product of the conduction time and refractory period (Smeets et al., 1986; Wijffels et al., 1995), is a determinant of re-entry. Changes in the expression and function of membrane ion channels (termed electrical remodeling) affect the cardiac conduction properties and refractory period, facilitating functional re-entry (Papadatos et al., 2002). The alteration of gap junction-specialized structures that couple myocytes (Kirchhoff et al., 1998; Jansen et al., 2010), the deposition of extracellular matrix components (Verheule et al., 2004; Krul et al., 2015), and cardiac enlargement (Eijsbouts et al., 2003; Vranka et al., 2007) are considered part of the structural remodeling that increases the chances of arrhythmia via anatomical re-entry mechanisms. Triggered activity refers to the impulse initiation resulting from depolarization of the membrane potential after the upstroke phase of the action potential (AP). Afterdepolarizations that occur during the repolarization phase of the AP are called early-afterdepolarizations (EADs), whereas those occurring after completion of the membrane repolarization are known as delayed-afterdepolarizations (DADs). The modulation of membrane ion channels that prolong the AP facilitates the appearance of EADs, whereas the appearance of DADs is commonly associated with altered intracellular $\mathrm{Ca}^{2+}$ homeostasis. Both increased $\mathrm{Ca}^{2+}$ overload conditions and Ryanodine receptor (RyR2) dysfunction (leakiness) promote spontaneous $\mathrm{Ca}^{2+}$ releases from the sarcoplasmic reticulum (SR) and activate the $\mathrm{Na}^{+} / \mathrm{Ca}^{2+}$ exchanger (NCX) in its forward mode. In this operating mode, a net inward $\mathrm{Na}^{+}$current $\left(\mathrm{I}_{\mathrm{ti}}\right)$ is present, which depolarizes the membrane and causes DADs. If these DADs are large enough, they can reach the threshold activation of the $\mathrm{Na}^{+}$current and generate a full arrhythmogenic AP. Both EADs and DADs are known as trigged activity and play an important role in initiating cardiac arrhythmias. There is compelling evidence that mitochondria play an important role in the generation of $\mathrm{Ca}^{2+}$-triggered arrhythmia, a process that requires an understanding of cellular $\mathrm{Ca}^{2+}$ fluxes.

The SR is the organelle in charge of storing and releasing $\mathrm{Ca}^{2+}$ into the cytosol. RyR2 opening occurs after a small initial amount of $\mathrm{Ca}^{2+}$ passes through L-type $\mathrm{Ca}^{2+}$ channels (LTCCs) in the sarcolemma in response to membrane depolarization during the AP (Bers, 2002). The sudden increase in cytosolic $\mathrm{Ca}^{2+}$ levels activates the myofibrils in sarcomeres, and contraction occurs. During the diastolic phase, $\mathrm{Ca}^{2+}$ is removed from the cytosol, and around $70 \%$ of total cytosolic $\mathrm{Ca}^{2+}$ is pumped back into the SR (Bassani et al., 1994) by the sarco/endoplasmic reticulum $\mathrm{Ca}^{2+}$ ATPase (SERCA). $\mathrm{Ca}^{2+}$ is extruded into the extracellular space by NCX activation, which counterbalances the entry of $\mathrm{Ca}^{2+}$ through LTCCs. Only a discreet quantity of cytosolic $\mathrm{Ca}^{2+}$ is removed by mitochondria (Bers, 2002), which occurs through a $\mathrm{Ca}^{2+}$ channel in the inner mitochondrial membrane (IMM) known as mitochondrial $\mathrm{Ca}^{2+}$ uniplex (mCU) (previously called the mitochondrial $\mathrm{Ca}^{2+}$ uniporter). Mitochondrial $\mathrm{Ca}^{2+}$ extrusion is carried out by the mitochondrial NCX, which possesses slow kinetics compared to the $\mathrm{mCU}$, allowing the accumulation of this ion in the mitochondria. The local SRMitochondria $\mathrm{Ca}^{2+}$ flux is facilitated by anchoring proteins that anchor both organelles (Kohlhaas and Maack, 2013). The dependence on $\mathrm{Ca}^{2+}$ import in mitochondrial reactive oxygen species (ROS) production and energetics highlights the importance of coordinated regulation between $\mathrm{SR} \mathrm{Ca}^{2+}$ fluxes and mitochondrial homeostasis. In this review, we focus on describing the cross signals that occur between these two organelles and how dysregulation of this intertwined signaling may be involved in altered cardiac rhythms.

\section{THE MITOCHONDRIA-SR INTERCONNECTION: PROXIMITY ENABLES CROSSTALK}

The structure of the cardiomyocyte has been extensively reviewed (Eisner et al., 2017), and emphasis has been placed on the proximity of T-tubules, where LTCCs reside, with the terminal cisternae of the SR, where RyR2s are more concentrated. The approximate distance between both structures has been calculated to be only about $15 \mathrm{~nm}$ (Scriven et al., 2013). This distance is what enables the two structures to react to each other's activation. This unit, comprised of a T-tubule with its corresponding terminal cisternae of the SR, is called a dyad; it is considered the functional unit of the heart and is in charge of excitation-contraction-coupling (ECC). Nonetheless, because of its proximity, mitochondria could also be regarded as part of this functional unit, as they also play a role in responding to stimulation and producing a proper contractile response. Fluorescence (Friedman et al., 2011) and electron microscopy (Csordás et al., 2006) techniques have been used to reveal physical interactions between these organelles, with protein-like structures linking both membranes. Protein structures between the mitochondria and the SR have been described elsewhere (Lopez-Crisosto et al., 2017; Martinvalet, 2018; Giorgi et al., 2018). Several structures have been found to create bridges between the organelles, securing proximity (Figure 1). The complexity of SR-mitochondria bridging proteins is high, so the removal of individual structures could be compensated for by other components. Describing the interactions of each element found to link both organelles in detail is out of the scope of 
this review, but a brief description will be provided. The first of such structures is the $\mathrm{Ca}^{2+}$ channel inositol-3-phosphate receptor (IP3R), joined to the mitochondrial voltage-dependent anion channel (VDAC) through protein GRP75 (Szabadkai et al., 2006). This communication enables the rapid movement of $\mathrm{Ca}^{2+}$ ions from the SR into the mitochondrial intermembrane space when IP3 is released through the protein kinase C (PKC) pathway. Similarly, IP3R2 has been described as binding with the FUN14 domain containing 1 (FUNDC1) to modulate SR $\mathrm{Ca}^{2+}$ release (Wu et al., 2017). The VDAC has also been described as having physical interactions with the RyR2, which, coupled with mCU co-localization with the RyR2, helps explain how mitochondrial $\mathrm{Ca}^{2+}$ transport is possible (Kohlhaas and Maack, 2013). Other structures involved in maintaining a connection are the SR vesicle-associated membrane protein-associated protein $\mathrm{B} / \mathrm{C}$ (VAPB), whose function is not fully understood, although it has been shown to regulate $\mathrm{Ca}^{2+}$ transport between both organelles (De Vos et al., 2012). The mitochondrial protein tyrosine phosphatase-interacting protein 51 (PTPIP51) seems to have more structural functions (Stoica et al., 2014). The ERmitochondria encounter structure (ERMES) is a protein complex characterized in yeast that bridges both organelles and has diverse biological functions. Although no homolog structure has been described in mammals, an ortholog of one of its components, PDZ domain-containing protein 8 (PDZD8), was described recently (Giorgi et al., 2018). Other structures, such as Mitofusin2, a protein involved in mitochondrial dynamics, have also been described as being able to form dimers that bridge both organelles (de Brito and Scorrano, 2008), presumably to organize mitochondrial dynamics. Similarly, SR protein inverted formin 2 (INF2) serves as an anchor for actin filaments to reach the mitochondria, thereby providing scaffolding for mitochondrial constriction in mitochondrial dynamics (Korobova et al., 2013; Manor et al., 2015). These connections help maintain the close gap between mitochondria and the SR. Intermyofibrillar mitochondria have been measured as close as $33 \mathrm{~nm}$ to the RyR2 in the SR and as far away as $188 \mathrm{~nm}$ (Ramesh et al., 1998). This proximity enables the existence of a microdomain where secure communication can take place. For instance, cellular $\mathrm{Ca}^{2+}$ concentrations vary from about 100-500 nM globally between resting and peak concentrations in the AP (Bers, 2002). Furthermore, within the dyadic cleft, $\mathrm{Ca}^{2+}$ levels can get as high as $100 \mu \mathrm{M}$ at the periphery when the RyR2 releases $\mathrm{Ca}^{2+}$ from the SR, stimulated by LTCCs (Langer and Peskoff, 1996). These high levels of $\mathrm{Ca}^{2+}$ are maintained for about $10 \mathrm{~ms}$, affecting a region of about $2 \mu \mathrm{m}$, although not in the same concentrations, before descending as $\mathrm{Ca}^{2+}$ diffuses to other cellular regions (Cheng and Lederer, 2008). This localization places the mitochondria well within reach of accessing high local $\mathrm{Ca}^{2+}$ concentrations. Although $10 \mathrm{~ms}$ may not seem very long, this is enough time for $\mathrm{Ca}^{2+}$ to be transported through the IMM by the mCU into the mitochondrial matrix. Two models of the mitochondrial response to changes in cytosolic $\mathrm{Ca}^{2+}$ levels have been described (O'Rourke and Blatter, 2009). The first one, originally proposed by Crompton (Crompton, 1990), indicates that the mitochondrial $\mathrm{Ca}^{2+}$ concentration increases slowly and gradually with a faster AP firing rate until influx and efflux are balanced entirely, and a new steady state is achieved. However, slow changes in the mitochondrial $\mathrm{Ca}^{2+}$ concentration may not be able to stimulate ATP production fast enough to meet immediate metabolic needs. Subsequently, beat-to-beat changes in mitochondrial $\mathrm{Ca}^{2+}$ are slight, and the energetic requirements of integrated mitochondrial $\mathrm{Ca}^{2+}$ transport are marginal. The other model describes mitochondria as having the ability to comprehensively sense rapid cytosolic $\mathrm{Ca}^{2+}$ changes, presenting with oscillations on a beat-to-beat basis (O'Rourke and Blatter, 2009). This process would imply that mitochondria can not only rapidly internalize but also extrude $\mathrm{Ca}^{2+}$ ions. In consolidating both models, a mitochondrial $\mathrm{Ca}^{2+}$ transient was described by Lu et al. (2013), which has some key differences when compared to its cytosolic counterpart. As the firing rate of the AP increases, a decrease in amplitude maintains a more stable concentration throughout the whole AP by slowly increasing diastolic $\mathrm{Ca}^{2+}$ concentrations, although a faster decline was also noted compared to baseline after stimulating with a catecholamine analog (Lu et al., 2013). It is estimated that this happens because mitochondrial structures pump $\mathrm{Ca}^{2+}$ into the cytosol more slowly, primarily the mitochondrial $\mathrm{Na}^{+} / \mathrm{Ca}^{2+}$ exchanger (mNCX), as opposed to the combined pumping force of SERCA in the SR and the sarcolemmal NCX. While it has been calculated that the percentage of cellular $\mathrm{Ca}^{2+}$ taken up by mitochondria is modest (Bassani et al., 1994), it can have significant effects on excitation-contraction-energetics coupling (ECEC). The main reason is that by increasing mitochondrial $\mathrm{Ca}^{2+}$ concentration, dehydrogenases from the Krebs cycle change to a more active form. In turn, these dehydrogenases produce more high-energy products $\left(\mathrm{NADH}^{+}\right.$and $\left.\mathrm{FADH}_{2}\right)$ for the electron transport chain (ETC) to use as substrates and generate the mitochondrial membrane potential $(\Delta \Psi \mathrm{m})$ and subsequent ATP synthesis under energy-demanding states, such as when adrenergic stimulation takes place (Fernandez-Sada et al., 2014; Kwong et al., 2015). This ATP is then transported to the cytosol, where it is used by the sarcomere to relax its myofibrils and, equally necessary, used by a wide range of pumps to maintain ion balance. One of these pumps is SERCA, which returns most $\mathrm{Ca}^{2+}$ ions, about $70 \%$ (Bassani et al., 1994), into the SR after the AP finishes and reestablishes the basal $\mathrm{Ca}^{2+}$ concentration. This is just one example of how mitochondria can communicate with the SR under demanding and stressful conditions, according to ECEC.

\section{MITOCHONDRIAL $\mathrm{CA}^{2+}$ SIGNALING AND CARDIAC ARRHYTHMIA}

High cytosolic $\mathrm{Ca}^{2+}$ concentrations trigger mitochondrial $\mathrm{Ca}^{2+}$ transport by the mCU. Under homeostatic conditions, this process is finite, and $\mathrm{Ca}^{2+}$ can slowly be transported back into the cytosol by the mNCX. However, if $\mathrm{Ca}^{2+}$ cannot be extruded out of the mitochondria before more $\mathrm{Ca}^{2+}$ enters the mCU, then $\mathrm{Ca}^{2+}$ overload ensues. High mitochondrial $\mathrm{Ca}^{2+}$ concentrations cause higher ROS production, loss of mitochondrial membrane integrity with subsequent $\Delta \Psi \mathrm{m}$ loss, and mPTP opening (Kazak et al., 2017). This last event is an initial step in the signaling 


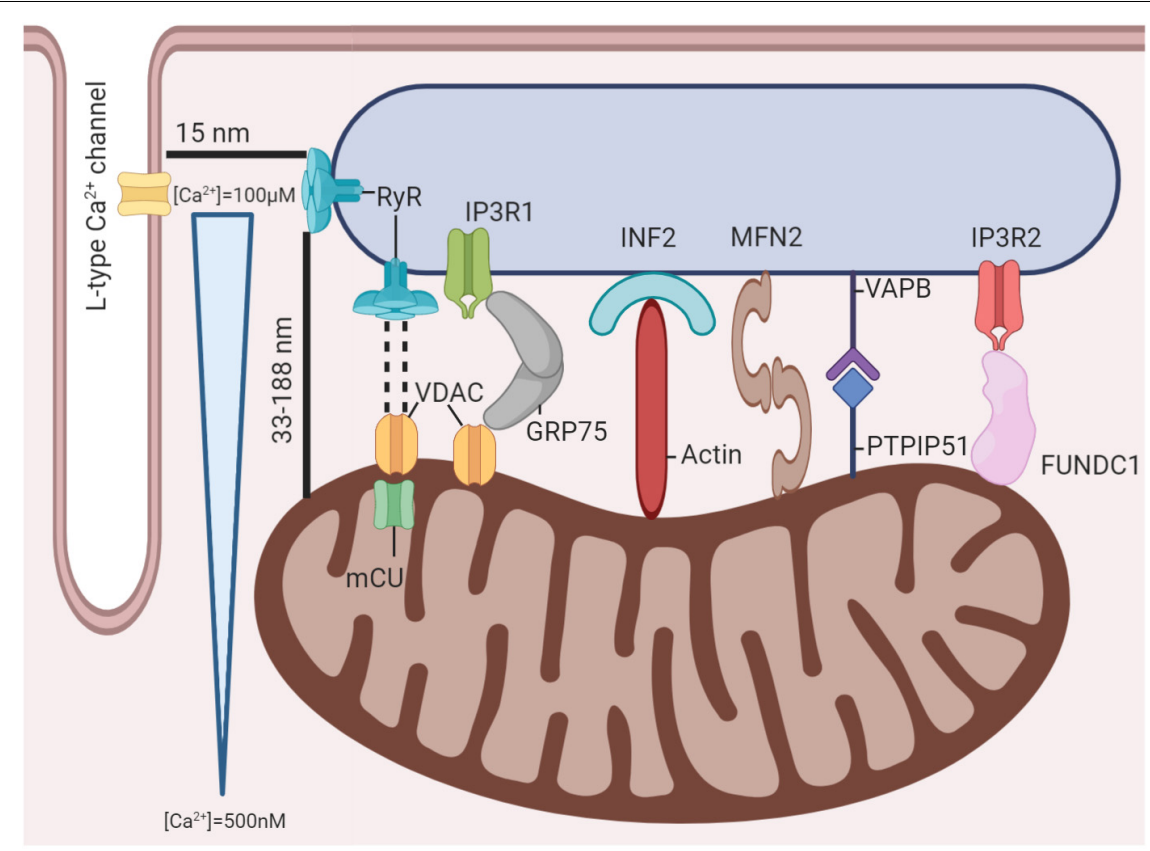

FIGURE 1 | Mitochondria-SR-T tubule microdomain. Several protein structures have been demonstrated to exist between mitochondria and the SR. These structures maintain proximity between the organelles and enable mitochondria to experience significant $\mathrm{Ca}^{2+}$ fluctuations with each AP despite the global cardiomyocyte's $\mathrm{Ca}^{2+}$ concentration remaining relatively unchanged.

cascade for the mitochondrial-mediated apoptosis pathway, causing further alterations in heart rhythmicity and organization at a cellular level. Whether SR or mitochondrial dysfunction causes the initial $\mathrm{Ca}^{2+}$ overload is debatable. Nonetheless, it is a safe bet to argue that this process may be self-maintained. Based on murine models of HF and AF associated with RyR2, as well as atrial samples from patients with chronic AF, the Marks group has reported that increased RyR2-mediated leakage is a potential mechanism for augmented mitochondrial $\mathrm{Ca}^{2+}$ content and ROS production. Post-translational analysis has revealed increased RyR2 oxidation and dissociation of the RyR2 inhibitory protein, calstabin2 or FKBP, which may further exacerbate RyR2 leakage. Thus, a feedback loop mechanism in $\mathrm{Ca}^{2+}$ signaling between the SR and mitochondria promotes intracellular $\mathrm{Ca}^{2+}$ mishandling. The SR-mitochondria interconnection in $\mathrm{Ca}^{2+}$ fluxes may offer the possibility of targeting mitochondrial $\mathrm{Ca}^{2+}$ overload to ameliorate RyR2-mediated leakage (Santulli et al., 2015; Xie et al., 2015). The importance of mitochondria in this vicious cycle is exemplified in a recent study conducted by Hamilton et al. (2020), who demonstrated that mitochondrial ROS targeting ameliorates RyR2 leakiness in a mouse model of catecholaminergic polymorphic ventricular tachycardia (CPVT). As part of the $\mathrm{Ca}^{2+}$-handling proteins, mitochondrial membrane components per se are susceptible to redox modulation, which contributes to altered $\mathrm{Ca}^{2+}$ homeostasis in the myocyte. This is the case for the mCU, whose oxidation at cysteine 97 activates the channel, leading to mitochondrial $\mathrm{Ca}^{2+}$ overload and ROS production (Dong et al., 2017). The impact of altered mitochondrial $\mathrm{Ca}^{2+}$ in membrane excitability and arrhythmia has also been investigated. Myocytes from non-ischemic $\mathrm{HF}$ showed increased mitochondrial $\mathrm{Ca}^{2+}$ transients, enhanced LTCC currents, AP prolongation, and the appearance of EADs (Xie et al., 2018). Cardiac activity monitored by telemetry has demonstrated QT prolongation and a high incidence of ventricular arrhythmia following the application of isoproterenol (ISO), a catecholamine analog.

A potential causal role of mitochondrial $\mathrm{Ca}^{2+}$ signaling in arrhythmia is illustrated by genetically and pharmacologically blocking the mCU. We previously reported that selective $\mathrm{mCU}$ inhibition has the therapeutic potential to prevent catecholamine-induced toxicity, as observed in HF (FernandezSada et al., 2014). In this regard, using mCU transgenic mice, two independent groups confirmed our findings of the mCU's critical role in matching the metabolic output during the adrenergic response (Kwong et al., 2015; Wu et al., 2015). Blocking the $\mathrm{mCU}$ with ruthenium red (RuR) or $\mathrm{Ru}$ $360 \mathrm{~min}$ before the induction of ischemia-reperfusion (I/R) injury prevents the appearance of ventricular arrhythmia in rat models (Garcia-Rivas Gde et al., 2006; Menezes-Rodrigues et al., 2020). In contrast, other studies have reported that enhancing $\mathrm{Ca}^{2+}$ fluxes between the SR and mitochondria using mitochondrial $\mathrm{Ca}^{2+}$ uptake enhancers is beneficial in preventing the appearance of ventricular arrhythmia associated with RyR2 dysfunction (Schweitzer et al., 2017). In atrial myocytes, the Blatter group has reported that inhibiting the mCU with Ru360 aggravates the $\mathrm{Ca}^{2+}$ alternans ratio and decreases the alternans' pacing threshold. These arrhythmogenic effects are restored when myocytes are treated with spermine, suggesting that mitochondrial $\mathrm{Ca}^{2+}$ uptake could be a potential candidate for AF treatment (Oropeza-Almazan and Blatter, 2020). Thus, the 
effect of blocking mitochondrial $\mathrm{Ca}^{2+}$ uptake, either protecting from or promoting arrhythmia, may be dependent on the cardiac condition associated with $\mathrm{Ca}^{2+}$ overload. For example, in I/R injury, where a massive and acute $\mathrm{Ca}^{2+}$ imbalance occurs, closing the mCU prevents mitochondrial damage, whereas stimulated $\mathrm{Ca}^{2+}$ transport in AF could ameliorate the emerging $\mathrm{Ca}^{2+}$ triggered arrhythmia by dissipating $\mathrm{Ca}^{2+}$ waves and lowering diastolic $\mathrm{Ca}^{2+}$ levels.

\section{MITOCHONDRIAL ENERGETIC DEBACLE IN CARDIAC ARRHYTHMIA}

About $90 \%$ of the heart's ATP is produced through oxidative phosphorylation in mitochondria (Harris and Das, 1991). Given that the heart beats continuously, cardiac muscle contraction is an extremely ATP-demanding process. Furthermore, mitochondria constitute about $25 \%$ of the volume of human cardiomyocytes, even more so in rats and mice $(\sim 30 \%)$ (Schaper et al., 1985), which reflects the organelle's relevance in cardiac tissue. Rising cytosolic $\mathrm{Ca}^{2+}$ levels during the AP stimulates $\mathrm{Ca}^{2+}$ transport into the mitochondrial matrix. This rising intramitochondrial $\mathrm{Ca}^{2+}$ concentration activates dehydrogenases involved in the Krebs cycle, which, in turn, supplies the ETC with $\mathrm{NADH}^{+}$and $\mathrm{FADH}_{2}$ to meet the cell's energetic demand (McCormack and Denton, 1990; Glancy and Balaban, 2012; Fernandez-Sada et al., 2014). In this sense, in cardiac cells, the $\mathrm{Ca}^{2+}$ signal is coupled with the energy demand through a process called ECEC. This process links $\mathrm{Ca}^{2+}$ handling and the contraction of the cardiomyocyte with relaxation and ATP production in mitochondria (Maack and O'Rourke, 2007). Energetic depletion can affect highly dependent ion channels and transporters that participate in proper intracellular $\mathrm{Ca}^{2+}$ handling. A prominent example of such a protein is SERCA, which needs ATP to transport $\mathrm{Ca}^{2+}$ into the SR during myocardium relaxation (Bers, 2002). Impairing SERCA's functioning reduces SR $\mathrm{Ca}^{2+}$ content and increases the cytosol's diastolic $\mathrm{Ca}^{2+}$ concentration. Another example of an ATP-driven protein function is sarcKATP, a potassium ion channel in the sarcoplasmic membrane that opens upon a low ATP/ADP ratio. High ATP/ADP ratios usually inhibit this channel, which limits the reactivation of inward currents by prolonging the refractory period and avoiding functional re-entry. The O'Rourke lab recently proposed that sarcKATP channels are the means by which changes in the $\Delta \Psi \mathrm{m}$ cause altered cellular membrane excitability. In their work, metabolic dysfunction induced by the mitochondrial uncoupler carbonyl cyanide-p-trifluoromethoxyphenylhydrazone (FCCP) increased IKATP and shortened the AP and refractory period, leading to re-entry into myocyte monolayers. The proarrhythmogenic effects of FCCP are attenuated by the IKATP inhibitor glibenclamide (Zhou et al., 2014). Depleting mitochondrial ATP production may impact membrane excitability by promoting T-wave alternans. In the electrocardiogram (ECG), T-wave alternans are linked to ventricular arrhythmia and constitute a prominent arrhythmogenic mechanism in the settings of myocardial infarction and ischemia, conditions characterized by the depletion of mitochondrial ATP production. T-wave alternans reflect beat-to-beat variation in the action potential duration (APD) at a cellular level, which are known as APD alternans, and numerous studies have reported that they are coupled to intracellular $\mathrm{Ca}^{2+}$ release variations $\left(\mathrm{Ca}^{2+}\right.$ transient alternans). In an isolated rabbit heart, FCCP perfusion caused a higher incidence of APD and $\mathrm{Ca}^{2+}$ transient alternans, increased interventricular heterogeneity, impaired cardiac conduction velocity, and promoted ventricular arrhythmias in optical mapping studies. In this study, APD and $\mathrm{Ca}^{2+}$ alternans were also induced under ischemic conditions in rabbit heart preparation, validating the clinical relevance of blunted mitochondria energetics in cardiac arrhythmogenesis (Smith et al., 2013). In contrast, several other studies have reported that mitochondrial uncoupling proteins (UCPs) confer protection against cardiac arrhythmias in conditions of $I / R$. Subjecting cardiac UCP3 knockout (UCP3-/-) mice to I/R injury produced twofold larger infarcts, a high propensity for ventricular tachycardia, and excessive ROS generation when compared to WT mice. Pretreatment with FCCP prior to $\mathrm{I} / \mathrm{R}$ injury relieved cardiac stress in $\mathrm{UCP} 3^{-/-}$hearts and improved ventricular functioning (Ozcan et al., 2013). Thus, the balance between mitochondrial energetics preservation should be a target for better cardiac arrhythmia protection in cardiac ischemia. Mitophagy, the degradation of mitochondria by autophagy, is considered a protective mechanism against the damage that mitochondria experience during I/R injury. Adenoviral expression of the uncoupling protein 2 (UCP2) in the heart promoted mitochondria mitophagy in the settings of $I / R$ injury and offered cardiac output preservation comparable with ischemic preconditioning (Wu et al., 2019). The significance in cardiac arrhythmia was not addressed in this study, but it may be the case since another study reported that the genetic ablation of $\mathrm{UCP} 2^{-/-}$in mice resulted in AP and QT shortening, leading to a high propensity for ventricular arrhythmias (Larbig et al., 2017). In the setting of AF, high AP firing imposes a high demand for ATP in the atrial chambers, causing mitochondria remodeling, altered $\mathrm{Ca}^{2+}$ handling, and impaired atrial contraction. In atrial-like myocytes (HL-1 cells), 6-8 h of tachypacing caused dissipation of the $\Delta \Psi \mathrm{m}$ and mitochondrial network, as well as the loss of mitochondrial $\mathrm{Ca}^{2+}$ transients. Interestingly, treatment with SS31, a compound that accelerates the formation of ATP from ADP, protected against mitochondria remodeling, impaired respiration, and $\mathrm{Ca}^{2+}$ homeostasis (Wiersma et al., 2019). The AMP-activated protein kinase (AMPK) is a cellular energy sensor that activates when the AMP/ATP ratio increases. Expression of a mutant (T172D) of the AMPK $\alpha 1$-subunit in rat ventricular myocytes resulted in the impaired open-state inactivation of Nav1.5, which led to AP prolongation and a high incidence of EADs at the cellular level. Whether this arrhythmogenic mechanism operates in conditions of high metabolic stress, as in ischemic HF and AF genetic syndrome, has not been yet determined (Harada et al., 2012). Similarly, impaired cardiac metabolism has been associated with several other cellular changes that increase the risk of arrhythmia generation. ATP, while mainly used by pumps and contractile machinery, is also used as a second messenger. ATP by itself can 
bind with RyR2 or $\mathrm{Mg}^{2+}$, a RyR2 inhibitor, and lower the $\mathrm{Ca}^{2+}$ threshold needed to elicit a $\mathrm{Ca}^{2+}$-induced-Ca ${ }^{2+}$ release (Fill and Copello, 2002; Meissner, 2004). Low ATP would mean RyR2 inhibition with a subsequent deficient $\mathrm{Ca}^{2+}$ release. Impaired metabolism also increases phosphate levels, decrease ATP levels which, inhibit sarcolemmal $\mathrm{Na}^{+} / \mathrm{K}^{+}$ATPase and activate the glycolytic pathway with the conversion of pyruvate to lactic acid, lowering the cell's $\mathrm{pH}$. The low $\mathrm{pH}$ activates the sarcolemmal $\mathrm{Na}^{+} / \mathrm{H}^{+}$exchanger, increasing the cytosolic $\mathrm{Na}^{+}$concentration. $\mathrm{Na}^{+}$accumulation is further aggravated by less active $\mathrm{Na}^{+} / \mathrm{K}^{+}$ ATPase and the translocation of connexin hemichannels to the non-junctional membrane, which turn into non-selective cation channels during ischemia or metabolic inhibition. High cytosolic $\mathrm{Na}^{+}$levels thus stimulate $\mathrm{Ca}^{2+}$ extrusion from the mitochondrial matrix through the mNCX, further inhibiting ATP synthesis and increasing cytosolic $\mathrm{Ca}^{2+}$ levels by inhibiting the sarcolemmal NCX (Yang et al., 2015).

Translocating connexin hemichannels to a non-junctional membrane, transforming them into non-selective channels, can also initiate other signaling pathways that promote arrhythmias (Kim et al., 2019). Because subsarcolemmal mitochondria also express connexin 43 in their IMM (Boengler et al., 2009), their proximity to the sarcolemma could enable the transport of messengers, such as ATP and $\mathrm{Ca}^{2+}$, between compartments, which could increase the risk of cellular and mitochondrial $\mathrm{Ca}^{2+}$ overload and arrhythmias. Another channel with properties similar to connexin is pannexin 1 (Panx1). This channel is activated by cytoplasmic $\mathrm{Ca}^{2+}$ (Locovei et al., 2006) and ROS (Zhang et al., 2008) and has been reported to release ATP into the extracellular matrix upon activation in fibrotic processes (Dolmatova et al., 2012). ATP released into the medium by these channels activates $\mathrm{P} 2 \mathrm{X}$ and $\mathrm{P} 2 \mathrm{Y}$ receptors, or it can be rapidly converted to adenosine in the extracellular matrix, binding to its G-coupled receptor, A2AR, and inducing TGF- $\beta 1$ expression (Shaikh et al., 2016; Zhang J. et al., 2017). This cytokine then stimulates collagen deposition and fibrosis of the cardiac tissue (Cerrone et al., 2018), which is known to act as a physical barrier to electrical signaling conduction, facilitating re-entry and arrhythmia propagation.

\section{MITOCHONDRIAL REDOX IMBALANCE AND CARDIAC ARRHYTHMIA}

In mitochondria, ROS is produced when electrons flowing through the ETC react prematurely with oxygen before reaching complex IV to form radical superoxide. These unstable molecules can then transform into hydrogen peroxide by mitochondrial superoxide dismutase or into hydroxyl radicals if they encounter a metal with which to react (Alfadda and Sallam, 2012). These byproducts of the ETC, while having a relatively short half-life, can diffuse to nearby cellular compartments and have various effects on a wide range of proteins. In the mitochondria-SR microdomain, proteins, such as the RyR2 and SERCA, are possible sites of protein oxidation, as well as other proteins implicated in the AP propagation that also reside within or near the dyadic cleft, all of which can affect cellular $\mathrm{Ca}^{2+}$ handling.
The RyR2 contains at least 21 free cysteine residues per monomer deemed to be susceptible to oxidation (Nikolaienko et al., 2018). Anzai et al. (1998) reported that hydroxyl radicals increased the opening probability of RyR2 channels reconstituted in lipid bilayers, an effect that was reverted by the SH-reducing agent dithiothreitol (Boraso and Williams, 1994; Anzai et al., 1998). At the cellular level, exposing cardiac myocytes to ROS increased the RyR2-mediated leak in permeabilized isolated ventricular myocytes, further supporting an up-regulatory effect of ROS (Terentyev et al., 2008). In another study, the $\beta$-adrenergic stimulation of myocytes promoted ROS production, oxidized RyR2, and, along with the RyR2 phosphorylation, enhanced the appearance of $\mathrm{Ca}^{2+}$ waves. MitoTEMPO treatment ameliorated the incidence of $\mathrm{Ca}^{2+}$ waves, indicating that mitochondria play a role in the production of oxidative stress (Bovo et al., 2012).

The proper functioning of the RyR2 is assisted by associated proteins, including calstabin and the FKBP12.5 factor. This factor exerts intrinsic negative regulation of the RyR2 and disrupts the RyR2-FKBP interaction, resulting in SR $\mathrm{Ca}^{2+}$ leakage and atrial and ventricular arrhythmias (Wehrens et al., 2003; Sood et al., 2008). The RyR2-FKBP association is redox-regulated (Zissimopoulos et al., 2007). As mentioned previously, highly oxidized levels of RyR2 were associated with increased SR $\mathrm{Ca}^{2+}$ leakage and an increased propensity for AF in a mouse model of CPVT. RyR2 oxidation was correlated with FKBP depletion from the RyR2, providing the molecular basis for atrial arrhythmia. Notably, the pharmacological stabilization of RyR2FKBP binding prevented diastolic spontaneous $\mathrm{Ca}^{2+}$ releases in isolated atrial myocytes and decreased the propensity for AF in vivo (Shan et al., 2012). Disruption of the RyR2-FKBP interaction by oxidative stress has also been identified as the mechanism by which palmitoyl-carnitine (PC) promotes RyR2 leakage and, subsequently, cardiac arrhythmia (Roussel et al., 2015). In this study, the measurement of ROS production by MitoSox showed increased mitochondrial superoxide amounts in myocytes pre-exposed to PC, suggesting that mitochondrial ROS reduces RyR2-FKBP stability.

Oxidative stress also affects the RyR2 channel's activity by activating $\mathrm{Ca}^{2+}$ calmodulin kinase II (CaMKII) (Erickson et al., 2008). The clinical significance of CaMKII oxidation in atrial arrhythmogenesis was first explored by Mark E. Anderson's group. They reported an increase in oxidized CaMKII levels in atria from patients with $\mathrm{AF}$, and the causality of $\mathrm{AF}$ was provided in a mouse model infused with the pro-oxidant hormone angiotensin II. After 3 weeks of treatment, angiotensin caused CaMKII oxidation, promoted SR $\mathrm{Ca}^{2+}$ leakage in isolated atrial myocytes and increased in vivo susceptibility to AF. The genetic inhibition of CaMKII oxidation reduced the propensity for AF, suggesting that CaMKII acts as a coupler between oxidative stress and atrial arrhythmia (Purohit et al., 2013). In line with these findings, Zhang et al. (2014) reported that the overexpression of NADPH oxidase 4 (NOX4) in zebrafish embryos resulted in increased superoxide production, CaMKII activation, and cardiac arrhythmia. Interestingly, MitoTEMPO treatment prevented NOX4-induced $\mathrm{H}_{2} \mathrm{O}_{2}$ production, indicating that mitochondrial oxidative stress plays a role in cardiac arrhythmia. 
The SERCA pump plays a critical role in myocyte relaxation by promoting the recapture of $\mathrm{Ca}^{2+}$ by the SR. This pump is also subjected to oxidative regulation. Cys674 is identified as a critical target for oxidative modification, including oxidation (Qin et al., 2013) and sulfonylation (Hobai et al., 2013). The exposure of an SR membrane preparation to $\mathrm{H}_{2} \mathrm{O}_{2}$ demonstrated that ROS slows down the recapture of $\mathrm{Ca}^{2+}$, which is consistent with an inhibitory effect (Qin et al., 2013). Loss of SERCA function caused by oxidative stress is, in part, responsible for diastolic dysfunction in aging (Herraiz-Martínez et al., 2015), inherited (Robinson et al., 2018) or acquired (Beuckelmann and Erdmann, 1992) cardiac conditions, and metabolic dysregulation (Bugger and Abel, 2014). Suppressed SERCA pump activity prolongs the $\mathrm{Ca}^{2+}$ spark duration life (O'Neill et al., 2004), increasing the chances of reaching the NCX and inducing triggered activity. The role of SERCA oxidation in cardiac arrhythmia in the setting of mitochondrial dysfunction deserves further exploration. As part of the $\mathrm{SR} \mathrm{Ca}^{2+}$-handling proteins, ROS is implicated in the development of cardiac substrates and triggers by causing the direct oxidation of membrane ion channels and structural elements or by indirectly targeting signaling pathways that modulate their function and expression. These topics have been extensively reviewed, and we would like to refer readers to excellent reviews in the area (Zima and Blatter, 2006; Takimoto and Kass, 2007; Jeong et al., 2012; Rutledge and Dudley, 2013).

Aging is a complex multi-factorial process characterized by a progressive decline in the organ function. Mitochondrial dysfunction also increases with aging and the resulting accumulation of ROS may contribute to the aging process by gradually damaging cellular components, a theory known as "the free radical theory of aging" proposed by Harman (1956). Aging is a primary risk factor for $\mathrm{AF}$, the most common arrhythmia in the elderly population. AF may be the result of the accumulation of damaged biomolecules by mitochondrial oxidative stress over the years being the mitochondrial DNA (mtDNA) one of the exposed targets. Analysis of the mtDNA integrity in atrial samples from patients with persistent AF revealed deletion of the $4977 \mathrm{bp}$, as well as a higher mitochondrial content of 8-OHdG compared with control patients, suggesting DNA damage by oxidative stress (Lin et al., 2003). A connection between oxidative stress and the pathophysiology of persistent AF may also have a genetic basis which is exemplified in the study conducted by Kim et al. (2003) who compared the transcriptional profile between control patients and patients with chronic AF using cDNA microarrays. Among those upregulated genes in atrial samples from AF patients, five were encoding enzymatic systems associated with ROS production.

Pulmonary vein (PV) dysregulation is involved in the initiation of AF and a study demonstrated that the reduction of the atrial arrhythmia burden in patients undergoing isolation of PVs correlated with significantly lower plasma levels of oxidative markers (advanced glycation end-products and thiobarbituricacid reacting substances) 9 months after the intervention (Böhm et al., 2016). From the experimental side, Wongcharoen et al. (2007) reported that PVs isolated from aged rabbits showed more depolarized resting membrane potentials and larger amplitude of EADs compared young PV preparations. Whether the high cellular arrhythmogenicity in aged PVs is linked to oxidative stress remains to be elucidated. Atrial remodeling, which contributes to the progression of permanent $\mathrm{AF}$, also increases with aging and animal studies support a causal role for oxidative stress. In this regard, pioglitazone, an activator of the peroxisomeactivated receptor gamma (PPAR- $\gamma$ ), decreased atrial fibrosis, NADPH oxidase subunits p22phox and gp91phox expression and the duration of pacing-induced AF in aged rats (Xu et al., 2012).

Aging regulates mitochondrial $\mathrm{Ca}^{2+}$ dependent processes including apoptosis and energetics by directly affecting mitochondrial components or their spatial relationship to the SR. For instance, the pharmacological inhibition of mPTP is reduced in hearts from senescent rats compared to younger animals when subjected to ischemia/reperfusion injury (Zhu et al., 2010). On the other hand, decreased mitochondrial $\mathrm{Ca}^{2+}$ uptake occurs in old isolated cardiomyocytes which correlated with lower mitochondrial $\mathrm{NAD}(\mathrm{P}) \mathrm{H} / \mathrm{NAD}\left(\mathrm{P}^{+}\right)$ratio in response to electrical pacing. Interestingly, immunolabelling of permeabilized myocytes with anti-RyR and anti-VDAC revealed an age-dependent reduction of the fraction of the RyR2 overlapping with VDAC, whereas quantitative redox proteomics showed augmented oxidation of VDAC proteins. Thus, oxidation of mitochondrial membrane components may contribute to the disruption of the spatial proximity between mitochondria and the SR, which may cause defective $\mathrm{Ca}^{2+}$ dependent mitochondrial energetics (Fernandez-Sanz et al., 2014). Which in turn others have appointed this increase in mitochondrial ROS to dysfunctional mitochondria which oxidize the RyR2, developing an increased risk of arrhythmia (Cooper et al., 2013). This increased arrhythmia risk was reduced by MitoTEMPO (Murphy et al., 2019).

\section{MITOPHAGY IN CARDIAC ARRHYTHMIA}

Since proper mitochondrial functionality is needed to prevent potentially damaging environments that predispose cardiac tissue to arrhythmias, defective remodeling of these organelles is also a potential mechanism for mitochondrial dysfunction with subsequent ROS generation and $\mathrm{Ca}^{2+}$ mishandling. Such is the case in the aging heart, were defective mitophagy has been described to ensue. This has been shown to cause accumulation of dysfunctional mitochondria and spontaneous $\mathrm{Ca}^{2+}$ release under adrenergic stimulation, which were prevented upon treatment with Torin1, an autophagy enhancer (Murphy et al., 2019). Another example is the SPRED2 deficiency murine model, an intracellular inhibitor of the ERK-MAPK pathway. SPRED2 ${ }^{-/-}$ mice have been described to develop cardiomyocyte hypertrophy, fibrosis, impaired excitability, and arrhythmias, which were associated with impaired mitophagy and the accumulation of defective mitochondria, along with increased ROS production, caspase 3 activity, and apoptosis, as this pathway seems to inhibit normal autophagy signaling. Selumetinib, a MAPK signaling inhibitor, restored autophagic flux in vivo, although no effects on arrhythmias were tested (Ullrich et al., 2019). Nonetheless, this proves the importance of proper mitochondrial remodeling to prevent damage accumulation and provides yet 


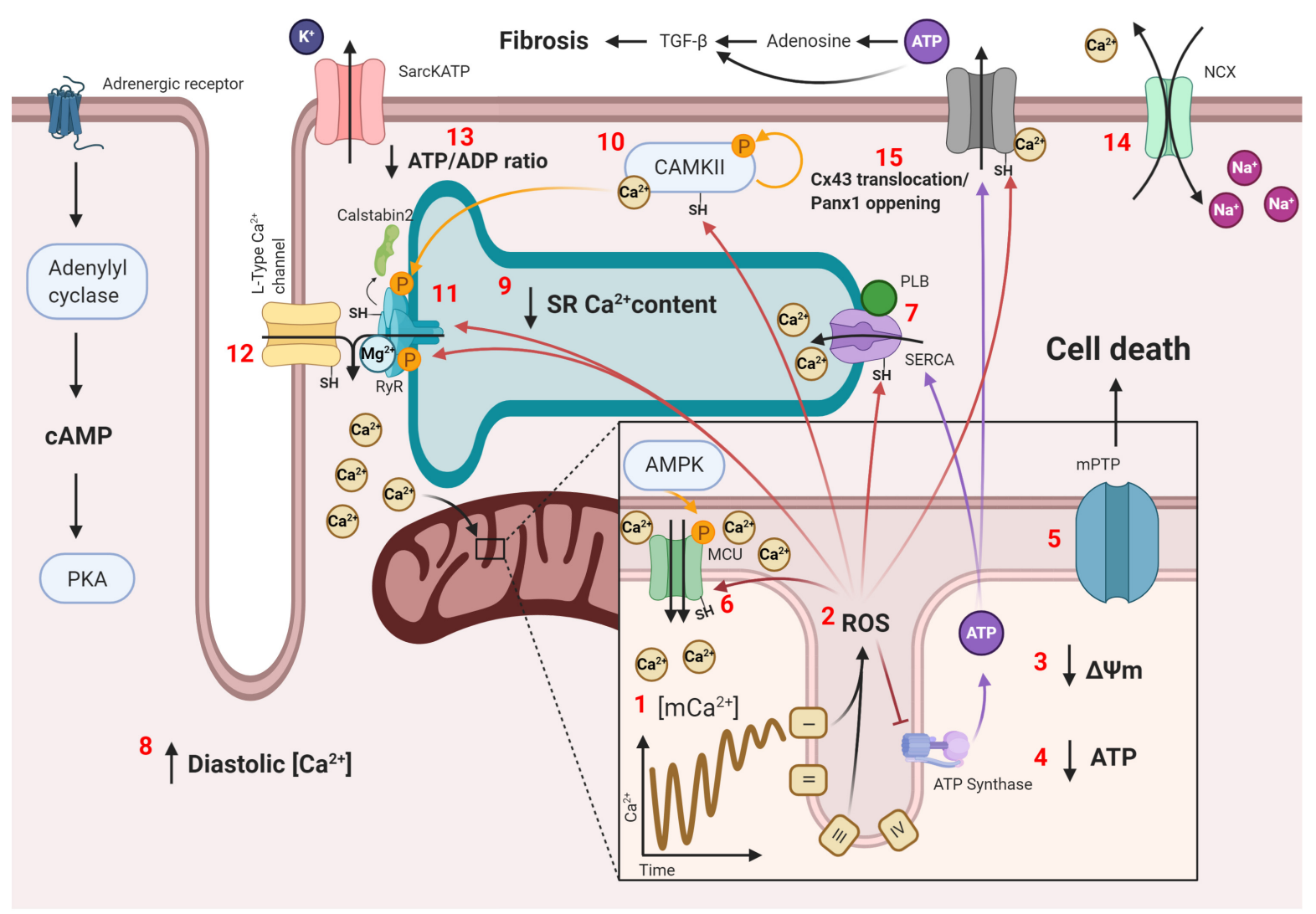

FIGURE 2 | Mitochondria-SR-T tubule communication in arrhythmogenesis. Conditions that favor $\mathrm{Ca}^{2+}$ overload, such as chronic adrenergic stimulation, cause the following: (1) increased $\mathrm{Ca}^{2+}$ transport through the $\mathrm{mCU}$ to the mitochondrial matrix, shortening mitochondrial $\mathrm{Ca}^{2+}$ transients' amplitude and stabilizing the mitochondrial $\mathrm{Ca}^{2+}$ concentration at a high level, which causes mitochondrial $\mathrm{Ca}^{2+}$ overload; (2) increased ROS production by mitochondrial structures, such as the ETC, with subsequent (3) loss of $\triangle \Psi \mathrm{m}$ and (4) decreased ATP production; (5) opening of the $\mathrm{mPTP}$, which initializes the cell death signal; (6) increased mCU activity by oxidation and, possibly, AMPK phosphorylation following a decline in the ATP concentration, which increases $\mathrm{Ca}^{2+}$ transport and ROS production; (7) decreased SERCA activity by oxidation and decreased PLB phosphorylation, which (8) increases the diastolic $\mathrm{Ca}^{2+}$ level and (9) decreases SR $\mathrm{Ca}^{2+}$ content, reducing contractility; (10) increased CAMKII activity by $\mathrm{Ca}^{2+}$ activation, oxidation, and autophosphorylation; (11) sensibilization of the RyR2 to luminal Ca ${ }^{2+}$ content with a reduced refractory state by oxidation and CAMKII hyperphosphorylation, with a subsequent unbinding of its modulating protein, calstabin2, and lower sensitivity to cytosolic $\mathrm{Ca}^{2+}$ by $\mathrm{Mg}^{2+}$ binding; (12) oxidation of LTCCs, which increases the amount of $\mathrm{Ca}^{2+}$ entering the cardiomyocyte upon activation; (13) opening of sarcKATP channels due to a reduced ATP/ADP ratio, which decreases the cardiomyocyte's bathmotropism and dromotropism; (14) increased activity of the NCX, which slowly depolarizes the cardiomyocyte and increases the risk of an unsolicited AP; (15) connexin 43 (Cx43) translocation in certain cardiac pathologies or pannexin 1 (Panx1) opening with $\mathrm{Ca}^{2+}$ or ROS transports ATP into the extracellular matrix, where it is transformed into adenosine, and both ATP and adenosine cause TGF- $\beta 1$ expression and fibrosis. All these changes combined produce, at the cellular level, $\mathrm{Ca}^{2+}$ mishandling with subsequent low contractility and spontaneous contraction that can propagate to neighboring cardiomyocytes and, at the tissular level, patches of slow conduction by reduced dromotropism or collagen deposition caused by cardiomyocyte cell death and fibrosis, which enable possible re-entry zones for sustained arrhythmias.

another possible therapeutic target, mitochondrial dynamics. The term mitochondrial dynamics refers to the changes by which individual mitochondria go through the process of fusion and fission within the cell to isolate damaged mitochondria or to better couple the generation of $\Delta \Psi \mathrm{m}$ to ATP synthesis, respectively. This is important in certain cardiac pathologies, such as HF. Pressure-overload HF murine models have been described to have an mitophagic blockade, which causes damaged mitochondria to accumulate and mitochondrial dysfunction to ensue (Yu et al., 2018). Regarding the different states of the mitochondrial network, fused mitochondria predomination has been described to be beneficial for cardiac function (Wai et al., 2015; Qin et al., 2020), as they are better at ATP synthesis and have lower ROS generation. Following this train of thought, inhibiting fission, promoting fusion, or maintaining an adequate mitophagy profile could potentially protect cardiac electrical signaling and organization. A summary with the presented mechanisms of arrhythmias are shown in Figure 2.

\section{NOVEL TARGETS IN SR-MITOCHONDRIA INTERCONNECTION AS POTENTIAL ANTI-ARRHYTHMIC DRUGS}

The development of new therapeutics could break the vicious cycle of $\mathrm{Ca}^{2+}$ overload, mitochondrial dysfunction, and ROS 
TABLE 1 | Summary of therapeutic strategies used to prevent arrhythmogenic events and their main findings.

\begin{tabular}{|c|c|c|c|c|c|}
\hline $\begin{array}{l}\text { Therapeutic } \\
\text { target }\end{array}$ & Compound & Mechanism of action & Models used & Main findings & References \\
\hline \multirow[t]{6}{*}{$\mathrm{mCU}$} & $\mathrm{Ru}_{360}$ & $\begin{array}{l}\text { Prevents mitochondrial } \\
\mathrm{Ca}^{2+} \text { overload by inhibiting } \\
\text { mitochondrial } \mathrm{Ca}^{2+} \\
\text { transport }\end{array}$ & $\begin{array}{l}\text { Pacing-induced VF model; } \\
\text { I/R murine model; NI-HF } \\
\text { murine model; pressure } \\
\text { overload HF model }\end{array}$ & $\begin{array}{l}\text { Reverted VF into VT; prevented } \\
\text { arrhythmias upon reperfusion; } \\
\text { prevented DADs in isolated } \\
\text { cardiomyocytes; and VF when } \\
\text { challenged with ISO; better } \\
\text { mitophagy profile }\end{array}$ & $\begin{array}{l}\text { Kimura et al., 2005; } \\
\text { Garcia-Rivas Gde et al., } \\
\text { 2006; Hamilton et al., } \\
\text { 2018; Xie et al., 2018; Yu } \\
\text { et al., } 2018\end{array}$ \\
\hline & Efsevin & $\begin{array}{l}\text { VDAC2 enhancer, increases } \\
\mathrm{Ca}^{2+} \text { transport into } \\
\text { mitochondria }\end{array}$ & Zebra fish cardiac tissue & $\begin{array}{l}\text { Prevented spark propagation } \\
\text { into } \mathrm{Ca}^{2+} \text { waves }\end{array}$ & $\begin{array}{l}\text { Shimizu et al., 2015; } \\
\text { Schweitzer et al., } 2017\end{array}$ \\
\hline & Kaempferol & $\begin{array}{l}\text { mCU enhancer, increases } \\
\mathrm{Ca}^{2+} \text { transport into } \\
\text { mitochondrial matrix }\end{array}$ & $\begin{array}{l}\text { CPVT murine model and } \\
\text { iPSC-derived human } \\
\text { cardiomyocytes }\end{array}$ & $\begin{array}{l}\text { Prevented diastolic } \mathrm{Ca}^{2+} \text { waves } \\
\text { and spontaneous AP in both } \\
\text { models as well as VT in vivo }\end{array}$ & Schweitzer et al., 2017 \\
\hline & Spermine & $\begin{array}{l}\text { mCU enhancer, increases } \\
\mathrm{Ca}^{2+} \text { transport into } \\
\text { mitochondrial matrix }\end{array}$ & $\begin{array}{l}\text { Tachypacing protocol in } \\
\text { atrial rabbit cardiomyocytes }\end{array}$ & $\begin{array}{l}\text { Lowered } \mathrm{Ca}^{2+} \text { transient } \\
\text { alternans }\end{array}$ & $\begin{array}{l}\text { Oropeza-Almazan and } \\
\text { Blatter, } 2020\end{array}$ \\
\hline & CGP-37157 & $\begin{array}{l}\mathrm{mNCX} \text { inhibitor, reduces } \\
\mathrm{Ca}^{2+} \text { transport out of the } \\
\text { mitochondria }\end{array}$ & Porcine model of I/R & $\begin{array}{l}\text { Suppressed ventricular } \\
\text { arrhythmias, lowered J point } \\
\text { depression and expedited ST } \\
\text { segment resolution }\end{array}$ & Sventzouri et al., 2018 \\
\hline & Cariporide & $\begin{array}{l}\mathrm{N}^{+} / \mathrm{H}^{+} \text {exchanger inhibitor, } \\
\text { potential regulator of } \\
\text { mitochondria uptake }\end{array}$ & $\begin{array}{l}\text { I/R model; isolated } \\
\text { cardiomyocytes with } \\
\text { ouabain poisoning; } \\
\text { cardiomyocytes with } \\
\text { angiotensin II and } \\
\text { endothelin } 1 \text { administration }\end{array}$ & $\begin{array}{l}\text { Prevented reperfusion } \\
\text { arrhythmia, preserved } \\
\text { metabolic status; prevented } \\
\mathrm{Ca}^{2+} \text { overload, delayed mPTP } \\
\text { opening and hyper contracture; } \\
\text { reduced ROS production and } \\
\mathrm{Ca}^{2+} \text { induced swelling }\end{array}$ & $\begin{array}{l}\text { Sugiyama et al., 1999; } \\
\text { Toda et al., 2007; } \\
\text { Garciarena et al., } 2008\end{array}$ \\
\hline \multirow[t]{7}{*}{ mROS } & Glutathione & $\begin{array}{l}\text { ROS scavenger to prevent } \\
\text { ROS damage }\end{array}$ & I/R in isolated rat hearts & $\begin{array}{l}\text { Reduced reperfusion } \\
\text { arrhythmias }\end{array}$ & $\begin{array}{l}\text { Woodward and Zakaria, } \\
1985\end{array}$ \\
\hline & $\begin{array}{l}\text { Ascorbic } \\
\text { acid }\end{array}$ & $\begin{array}{l}\text { ROS scavenger to prevent } \\
\text { ROS damage }\end{array}$ & $\mathrm{I} / \mathrm{R}$ in isolated rat hearts & $\begin{array}{l}\text { Reduced reperfusion } \\
\text { arrhythmias; reduced lipid } \\
\text { peroxidation, preserved } \\
\text { mitochondria respiratory } \\
\text { function and improved survival }\end{array}$ & $\begin{array}{l}\text { Woodward and Zakaria, } \\
\text { 1985; Tsai et al., } 2011\end{array}$ \\
\hline & $\mathrm{N}$ - acetylcysteine & Glutathione precursor & $\begin{array}{l}\text { I/R in isolated rat and dog } \\
\text { hearts; murine model of } \\
\text { fatty acid accumulation }\end{array}$ & $\begin{array}{l}\text { Reduced reperfusion } \\
\text { arrhythmias, reduced infarct } \\
\text { size, improved mechanical } \\
\text { function recovery; Preserved } \\
\Delta \Psi \mathrm{m} \text { and Ca2+ transient } \\
\text { amplitude, reduced Ca2+ } \\
\text { sparks and prevented VT }\end{array}$ & $\begin{array}{l}\text { Qiu et al., 1990; Sochman } \\
\text { et al., 1990; Roussel et al., } \\
2015\end{array}$ \\
\hline & TEMPOL & $\begin{array}{l}\text { ROS scavenger to prevent } \\
\text { ROS damage }\end{array}$ & I/R in rat model & $\begin{array}{l}\text { Reduced arrhythmias, lipid } \\
\text { peroxidation, preserved } \\
\text { mitochondria respiratory } \\
\text { function and improved survival }\end{array}$ & Tsai et al., 2011 \\
\hline & MitoTEMPO & $\begin{array}{l}\text { ROS scavenger to prevent } \\
\text { ROS damage }\end{array}$ & $\begin{array}{l}\text { ISO-exposed isolated } \\
\text { rabbit cardiomyocytes; } \\
\text { CPVT murine model; HF } \\
\text { guinea pig model; human } \\
\text { end-stage HF } \\
\text { cardiomyocytes }\end{array}$ & $\begin{array}{l}\text { Partially prevented SR Ca2+ } \\
\text { leak, Ca2+ waves, and ROS } \\
\text { production; reduced ROS } \\
\text { generation with subsequent } \\
\text { RyR2 oxidation and leakage; } \\
\text { prevented sudden cardiac } \\
\text { death, ventricular arrhythmias } \\
\text { and remodeling; reduced RyR2 } \\
\text { hyperactivity and } \mathrm{Ca} 2+\text { waves }\end{array}$ & $\begin{array}{l}\text { Bovo et al., 2012; Ni et al., } \\
\text { 2016; Dey et al., 2018; } \\
\text { Dries et al., 2018; Hamilton } \\
\text { et al., } 2020\end{array}$ \\
\hline & $\begin{array}{l}\text { SS-31 and } \\
\text { SS-20 }\end{array}$ & $\begin{array}{l}\text { Most likely ROS scavenger } \\
\text { and ROS production } \\
\text { inhibitor }\end{array}$ & I/R model & $\begin{array}{l}\text { Reduced infarct size, severity, } \\
\text { and duration of arrhythmias and } \\
\text { lipid peroxidation }\end{array}$ & Cho et al., 2007 \\
\hline & $\begin{array}{l}\text { Quercetin / } \\
\text { quercetin- } \\
\text { filled } \\
\text { liposomes }\end{array}$ & $\begin{array}{l}\text { ROS scavenger to prevent } \\
\text { ROS damage }\end{array}$ & I/R murine and dog model & $\begin{array}{l}\text { Reduced arrhythmias and } \\
\text { platelet aggregation; prevented } \\
\text { peroxynitrite-induced } \\
\text { arrhythmias }\end{array}$ & $\begin{array}{l}\text { Xiao et al., 1993; Soloviev } \\
\text { et al., 2002; Lozano et al., } \\
2019\end{array}$ \\
\hline
\end{tabular}


TABLE 1 | Continued

\begin{tabular}{|c|c|c|c|c|c|}
\hline $\begin{array}{l}\text { Therapeutic } \\
\text { target }\end{array}$ & Compound & Mechanism of action & Models used & Main findings & References \\
\hline & Resveratrol & $\begin{array}{l}\text { ROS scavenger, sirtuin } \\
\text { activator }\end{array}$ & $\begin{array}{l}\text { I/R murine model; } \\
\text { cardiomyocytes from } \\
\text { human-induced pluripotent } \\
\text { stem cells from patients } \\
\text { with very long-chain } \\
\text { acyl-CoA dehydrogenase } \\
\text { deficiency }\end{array}$ & $\begin{array}{l}\text { Reduced reperfusion } \\
\text { arrhythmias and mortality, } \\
\text { decreased LDH and increased } \\
\text { NO levels; reduced DADs and } \\
\text { cytosolic Ca2+ levels as well as } \\
\text { normalizing APD by decreasing } \\
\text { fatty acid oxidation } \\
\text { intermediates }\end{array}$ & $\begin{array}{l}\text { Hung et al., 2000; } \\
\text { Knottnerus et al., } 2020\end{array}$ \\
\hline & $\mathrm{DTT}$ & $\begin{array}{l}\text { Restores reduced thiol } \\
\text { groups in oxidized proteins }\end{array}$ & $\begin{array}{l}\text { Isolated atrial } \\
\text { cardiomyocytes exposed to } \\
\text { TNF- } \alpha\end{array}$ & $\begin{array}{l}\text { Decreased transient amplitude, } \\
\text { spark frequency and duration, } \\
\text { and ROS production }\end{array}$ & Zuo et al., 2018 \\
\hline \multirow[t]{3}{*}{ mPTP } & CsA & Inhibits mPTP opening & $\begin{array}{l}\text { I/R murine model; chronic } \\
\text { AV block canine model; VF } \\
\text { murine model; rabbit I/R } \\
\text { model }\end{array}$ & $\begin{array}{l}\text { Reduced arrhythmias, cardiac } \\
\text { edema and cardiomyocyte } \\
\text { death; reduced hypertrophy } \\
\text { and arrhythmia susceptibility, } \\
\text { no changes in electrical } \\
\text { remodeling; no protection } \\
\text { found; CsA offered no } \\
\text { protection }\end{array}$ & $\begin{array}{l}\text { Arteaga et al., 1992; } \\
\text { Schreiner et al., 2004; } \\
\text { Brown et al., 2008; Ayoub } \\
\text { et al., } 2017\end{array}$ \\
\hline & $\begin{array}{l}\text { Bongkrekic } \\
\text { acid }\end{array}$ & $\begin{array}{l}\text { Inhibits ANT, reducing } \\
\text { Ca2+ overload and ROS } \\
\text { production }\end{array}$ & $\begin{array}{l}\text { Murine model of fatty acid } \\
\text { accumulation }\end{array}$ & $\begin{array}{l}\text { Preserved } \Delta \Psi \mathrm{m} \text { and } \mathrm{Ca} 2+ \\
\text { transient amplitude, reduced } \\
\text { ROS production, RyR2 } \\
\text { oxidation, Ca2+ sparks and } \\
\text { prevented VT }\end{array}$ & Roussel et al., 2015 \\
\hline & Octylguanidine & Inhibits mPTP opening & $\begin{array}{l}\text { I/R murine model; } \\
\text { hyperthyroid I/R murine } \\
\text { model }\end{array}$ & $\begin{array}{l}\text { Prevented arrhythmias and } \\
\text { edema, preserved blood } \\
\text { pressure, and mitochondrial } \\
\text { function; prevented arrhythmias } \\
\text { and preserved hemodynamic } \\
\text { parameters, mitochondrial } \\
\text { function, and reduced } \\
\text { inflammatory cytokines }\end{array}$ & $\begin{array}{l}\text { Parra et al., 2005; Pavón } \\
\text { et al., } 2009\end{array}$ \\
\hline \multirow[t]{2}{*}{ CAMKII } & $\mathrm{KN}-93$ & CAMKII inhibitor & $\begin{array}{l}\text { Genetic murine models: } \\
\text { Gain-of-function RyR2, } \\
\text { CAMKIl overexpression, } \\
\text { constitutively active CAN, } \\
\text { CPVT Diabetic } \\
\text { hyperglycemia murine } \\
\text { model Human } \\
\text { cardiomyocytes from } \\
\text { diabetic HF patients } \\
\text { Cardiomyocytes derived } \\
\text { from induced pluripotent } \\
\text { stem cells from CPVT } \\
\text { patients Trabeculae from } \\
\text { HF patients }\end{array}$ & $\begin{array}{l}\text { Prevented AF after fast pacing; } \\
\text { prevented arrhythmias after ISO } \\
\text { administration; decreased } \\
\text { premature contractions and } \\
\text { sparks; improved contractile } \\
\text { function }\end{array}$ & $\begin{array}{l}\text { Khoo et al., 2006; Chelu } \\
\text { et al., 2009; Sag et al., } \\
\text { 2009; Sossalla et al., 2010; } \\
\text { Liu et al., 2011; Di et al., } \\
\text { 2013; Erickson et al., } 2013\end{array}$ \\
\hline & AIP & CAMKII inhibitor & $\begin{array}{l}\text { CAMKII overexpressing } \\
\text { murine model; trabeculae } \\
\text { from HF patients; impaired } \\
\text { glucose tolerance murine } \\
\text { model }\end{array}$ & $\begin{array}{l}\text { Prevented afterdepolarization in } \\
\text { isolated cardiomyocyte; } \\
\text { improved contractile function; } \\
\text { prevented arrhythmias and } \\
\text { arrhythmogenic cellular events }\end{array}$ & $\begin{array}{l}\text { Sag et al., 2009; Sossalla } \\
\text { et al., 2010; Federico et al., } \\
2019\end{array}$ \\
\hline
\end{tabular}


TABLE 1 | Continued

\begin{tabular}{|c|c|c|c|c|c|}
\hline $\begin{array}{l}\text { Therapeutic } \\
\text { target }\end{array}$ & Compound & Mechanism of action & Models used & Main findings & References \\
\hline RyR2 & Dantrolene & RyR2 stabilizer & $\begin{array}{l}\text { CPVT KI murine model } \\
\text { Ex vivo rabbit heart Long } \\
\text { QT syndrome model Atrial } \\
\text { infarct sheep model } \\
\text { Cardiac arrest pig model } \\
\text { Isolated cardiomyocytes } \\
\text { from AF or endstage HF } \\
\text { patients Cardiomyocytes } \\
\text { from induced pluripotent } \\
\text { stem cells from CPVT } \\
\text { patients Clinical trial with } \\
\text { CPVT patients }\end{array}$ & $\begin{array}{l}\text { Prevented VT and decreased } \\
\text { spark frequency; reduction of } \\
\text { EADs and VT frequency; } \\
\text { prevented AF; same efficacy as } \\
\text { amiodarone to regain } \\
\text { spontaneous circulation and } \\
\text { hemodynamic stability in } \\
\text { cardiac arrest; reduced spark } \\
\text { frequency, Ca2+ waves, Ca2+ } \\
\text { leak and spontaneous } \\
\text { transients; reduced premature } \\
\text { ventricular beats }\end{array}$ & $\begin{array}{l}\text { Kobayashi et al., 2010; } \\
\text { Penttinen et al., 2015; } \\
\text { Hartmann et al., 2017; } \\
\text { Wiesmann et al., 2017; } \\
\text { Avula et al., 2018; } \\
\text { Frommeyer et al., } 2018\end{array}$ \\
\hline \multirow[t]{2}{*}{ Calstabin2 } & JTV519 & $\begin{array}{l}\text { Increases affinity of } \\
\text { calstabin2 to the RyR2 }\end{array}$ & $\begin{array}{l}\text { HF dog model KD } \\
\text { calstabin2 murine model } \\
\text { HEK293 with CPVT Ry } \\
\text { mutations in vitro model 3D } \\
\text { engineered heart tissue } \\
\text { with optical activated ion } \\
\text { channel model }\end{array}$ & $\begin{array}{l}\text { Abolished abnormal RyR2 } \\
\text { gating; prevented VT and } \\
\text { sudden cardiac death }\end{array}$ & $\begin{array}{l}\text { Kohno et al., 2003; Lehnart } \\
\text { et al., 2004a; Wehrens } \\
\text { et al., 2004; Lemme et al., } \\
2019\end{array}$ \\
\hline & S107 & $\begin{array}{l}\text { Increases affinity of } \\
\text { calstabin2 to the RyR2 }\end{array}$ & $\begin{array}{l}\text { CPVT murine models DMD } \\
\text { murine model } \\
\text { Cardiomyocytes derived } \\
\text { from induced pluripotent } \\
\text { stem cells from CPVT } \\
\text { patients CPVT analogue } \\
\text { nematode model }\end{array}$ & $\begin{array}{l}\text { Prevented arrhythmias and } \\
\text { seizures; reduced Ca2+ leak; } \\
\text { prevented DADs }\end{array}$ & $\begin{array}{l}\text { Lehnart et al., 2008; } \\
\text { Fauconnier et al., 2010; } \\
\text { Shan et al., 2012; Sasaki } \\
\text { et al., 2016; Fischer et al., } \\
2017\end{array}$ \\
\hline \multirow[t]{2}{*}{$\begin{array}{l}\text { Mitochondrial } \\
\text { dynamics }\end{array}$} & Mdivi-1 & $\begin{array}{l}\text { Mitochondrial fission } \\
\text { inhibitor }\end{array}$ & I/R murine model & $\begin{array}{l}\text { Reduced arrhythmias and } \\
\text { preserved hemodynamic } \\
\text { parameters }\end{array}$ & Maneechote et al., 2018 \\
\hline & M1 & $\begin{array}{l}\text { Mitochondrial fusion } \\
\text { promoter }\end{array}$ & I/R murine model & $\begin{array}{l}\text { Reduced arrhythmias and } \\
\text { preserved hemodynamic } \\
\text { parameters }\end{array}$ & Maneechote et al., 2019 \\
\hline
\end{tabular}

generation to substantially reduce the incidence of sudden cardiac death and disease progression, especially in high-risk patients, such as those with HF. Fortunately, there are a few candidates that may prove useful in doing so. A summary of such compounds can be reviewed in Table 1.

\section{Regulation of Mitochondrial $\mathrm{Ca}^{2+}$ Transport}

Considering that the $\mathrm{mCU}$ seems to be the central piece in the positive feedback cycle, mCU inhibition may be efficient in stopping the cycle and maintaining mitochondrial integrity. $\mathrm{Ru}_{360}$ is a potent $\mathrm{mCU}$ inhibitor. It is an oxygen-bridged small molecule with a dinuclear ruthenium center complexed with amine groups. This molecule was first fully described in 1998 and synthesized from a familiar component, RuR. RuR was first used as the mCU inhibitor to study mitochondrial $\mathrm{Ca}^{2+}$ dynamics but was found to be non-specific for the $\mathrm{mCU}$, as it has various effects on other cellular components. $\mathrm{Ru}_{360}$ was then described as being more potent (IC50 of 0.184 vs. $6.85 \mathrm{nM}$ for RuR) and having a much greater affinity for the mCU, with a $\mathrm{Kd}$ of about $0.34 \mathrm{nM}$. Doses of up to $10 \mu \mathrm{M}$ had no effect on SERCA, the RyR2, NCX, LTCCs, or myofibrils (Matlib et al., 1998; García-Rivas et al., 2005), which demonstrates its high specificity for the mCU and safety. In this sense, $\mathrm{Ru}_{360}$ treatment partially inhibited the $\mathrm{mCU}$, maintaining proper mechanical performance of the heart and mitochondrial matrix free- $\mathrm{Ca}^{2+}$ concentrations at basal levels, despite high concentrations of cytosolic $\mathrm{Ca}^{2+}$ after ischemia/reperfusion (I/R) injury (García-Rivas et al., 2005). In an open-chest rat model of $\mathrm{I} / \mathrm{R}$, a bolus of $\mathrm{Ru}_{360}$ given before ischemia significantly prevented the occurrence of ventricular fibrillation (VF) and hemodynamic dysfunction. $\mathrm{Ru}_{360}$ administration was associated with protecting mitochondria from depolarization by mPTP opening and thus preserved mitochondrial functioning (Garcia-Rivas Gde et al., 2006). $\mathrm{Ru}_{360}$ infusion also reverted the 
progression of pacing-induced sustained VF into ventricular tachycardia, a less severe form of arrhythmia, in an isolated heart model. This was associated with a reduction in the APD, refractory period, and slope of the APD restitution curves (Kimura et al., 2005).

Since then, $\mathrm{Ru}_{360}$ has been used to research the effects of inhibiting mitochondrial $\mathrm{Ca}^{2+}$ transport on various models of cardiac injury. It has already proved its efficacy in preventing EADs in ventricular myocytes in a non-ischemic HF model (Xie et al., 2018). It has also been shown to reduce VF in mice with thoracic aorta banding when presented with a catecholamine challenge. It is worth mentioning that inhibiting mitochondrial $\mathrm{Ca}^{2+}$ transport was related to less oxidized RyR2 in isolated cardiomyocytes, as opposed to other drugs that increase mitochondrial $\mathrm{Ca}^{2+}$ content, such as kaempferol, the mCU agonist, and CGP-37157, the mNCX inhibitor, which enhances RyR2 oxidation. Isolated cardiomyocytes also presented with improved $\mathrm{Ca}^{2+}$ homeostasis and less spontaneous $\mathrm{Ca}^{2+}$ waves (Hamilton et al., 2018). This further proves that mitochondrial $\mathrm{Ca}^{2+}$ overload and ROS generation can affect SR components and should be taken into consideration more fully. Finally, mitochondrial $\mathrm{Ca}^{2+}$ transport inhibition was described as useful in maintaining proper ECG signaling in a murine pressureoverload HF model by enhancing mitochondrial autophagy (Yu et al., 2018). It has been previously described that mitochondrial dynamics (i.e., fusion, fission, and mitophagy) are impaired in a wide range of cardiovascular diseases and could be another potential therapeutic target (Vásquez-Trincado et al., 2016). Unsurprisingly, mitophagy was found to be impaired in the HF model and inhibiting mitochondrial $\mathrm{Ca}^{2+}$ transport seemed to prevent the accumulation of dysfunctional mitochondria, although no clear explanation was given for why this happened. It appears that there is much more to be described regarding the affected mitochondrial signaling mechanisms when mitochondrial $\mathrm{Ca}^{2+}$ overload ensues.

Inhibiting mitochondrial $\mathrm{Ca}^{2+}$ transport seems to be a plausible therapeutic strategy to prevent arrhythmias. Nonetheless, there is evidence indicating that the opposite approach would be more effective. It has been observed that efsevin, a VDAC2 enhancer, allows better $\mathrm{Ca}^{2+}$ transport from the SR to mitochondria by interacting directly inside the channel pore (Wilting et al., 2020). This enhanced $\mathrm{Ca}^{2+}$ transport was observed to abolish $\mathrm{Ca}^{2+}$ spark propagation into $\mathrm{Ca}^{2+}$ waves and erratic contractions by limiting the $\mathrm{Ca}^{2+}$ spark's temporal and spatial boundaries, effectively acting as a " $\mathrm{Ca}^{2+}$ sponge" (Shimizu et al., 2015). Similarly, in a CPVT murine model, diastolic $\mathrm{Ca}^{2+}$ waves and spontaneous APs in isolated cardiomyocytes were prevented by enhancing mitochondrial $\mathrm{Ca}^{2+}$ transport via either efsevin or the mCU enhancer, kaempferol. Ventricular tachycardia was prevented in vivo, and similar results were found in iPSC-derived human cardiomyocytes (Schweitzer et al., 2017). Furthermore, enhancing mitochondrial $\mathrm{Ca}^{2+}$ content using the mNCX inhibitor CGP-37157 suppressed ventricular arrhythmias, lowered the depression of the J point during ischemia, and expedited ST-segment resolution after reperfusion in a porcine model of I/R (Sventzouri et al., 2018). Likewise, enhancing mitochondrial $\mathrm{Ca}^{2+}$ transport using the
$\mathrm{mCU}$ activator spermine in isolated rabbit atrial cardiomyocytes undergoing a tachypacing protocol to induce AF demonstrated a protective effect against $\mathrm{Ca}^{2+}$ transient alternans. However, inhibiting mCU Ca ${ }^{2+}$ transport with $\mathrm{Ru}_{360}$ lowered the threshold needed to achieve alternans and enhanced the severity of the alternans (Oropeza-Almazan and Blatter, 2020).

Similar to the approach of using a specific mCU inhibitor, using gene therapy to downregulate $\mathrm{mCU}$ expression is another feasible alternative. With the development of better protocols to direct gene therapy to specific tissues, it may be possible to knockout (KO) mCU expression using siRNA (OropezaAlmazan et al., 2017). Transgenic mice with mCU KO in cardiac tissue were shown to have less mPTP opening than their WT counterparts and appeared to have no problems when resting and upon being put on a treadmill. These mCU KO mice could even sprint for as long as their WT counterparts, provided that they were given a 30-min "warm-up" beforehand, as they were unable to do so acutely (Kwong et al., 2015). This finding correlates with the finding of similar mitochondrial basal $\mathrm{Ca}^{2+}$ levels between groups, while the $\mathrm{KO}$ group needed more time to accumulate $\mathrm{Ca}^{2+}$ after stress stimulation. Cardiomyocytes from the KO group also showed less mPTP opening compared to the WT group, and the KO hearts had a smaller affected area after I/R injury. Another study using mice with $\mathrm{mCU}$ knockdown in cardiac tissue showed that upon being submitted to a non-ischemic HF model, they presented with reduced $\mathrm{Na}^{+} / \mathrm{Ca}^{2+}$ exchange currents, decreased APD, no observed EADs, and a reduced incidence of VF compared to the WT group (Xie et al., 2018). Combining these findings with the recent development of nanometric particles used for drug delivery places this type of therapy in the possible near future, as it has been recently proven that siRNA can be packaged and protected from degradation while maintaining its biological effects in vitro studies. Furthermore, cariporide, an $\mathrm{N}^{+} / \mathrm{H}^{+}$exchange inhibitor, has proven useful in not only preventing arrhythmias in an $\mathrm{I} / \mathrm{R}$ model but also preserving the metabolic status of the heart (Sugiyama et al., 1999). At the mitochondrial level, cariporide has also been shown to have mitochondrial protective properties. In isolated ventricular myocytes, cariporide prevented mitochondrial $\mathrm{Ca}^{2+}$ overload upon ouabain administration. No effect was seen in preventing $\Delta \Psi \mathrm{m}$ reduction, although $\mathrm{mPTP}$ opening was delayed significantly. This translated the ouabain taking longer to cause hyper contracture, which would indicate global ATP depletion and, thus, more preserved mitochondrial functioning (Toda et al., 2007). Notably, cariporide decreased $\mathrm{Ca}^{2+}$-induced mitochondrial swelling, comparable to the effects of Cyclosporine A (CsA) (Garciarena et al., 2008). $\mathrm{N}^{+} / \mathrm{H}^{+}$ exchange inhibitors seem to be a promising therapeutic approach for cardioprotection, although they require further studies to elucidate their precise mechanism of action in regulating mitochondrial $\mathrm{Ca}^{2+}$ handling to better design a therapeutic strategy.

\section{Modulation of Redox Homeostasis Cellular ROS Scavengers}

Since increased mitochondrial ROS generation seems to be the initial insult that starts the positive feedback loop, antioxidants 
could be useful in boosting the myocardium's ROS defense pool. Since their first trials in animal models, ROS scavengers have had beneficial effects in preventing arrhythmias during I/R. Superoxide dismutase, glutathione, and ascorbic acid significantly reduced reperfusion arrhythmias in isolated rat hearts (Woodward and Zakaria, 1985). N-acetylcysteine, a precursor for glutathione synthesis, also demonstrated protection against reperfusion arrhythmias in isolated rat heart (Qiu et al., 1990) and dog models (Sochman et al., 1990) of I/R, reducing infarct size, preventing reperfusion arrhythmias, and improving the recovery of mechanical functioning during reperfusion. Another dog model of $\mathrm{I} / \mathrm{R}$ was infused with superoxide dismutase. High-dose infusion was correlated with a reduction in ventricular extrasystoles, improved regional contractile force, and increased blood flow during reperfusion (Kónya et al., 1992). Other non-specific antioxidants, such as ascorbic acid and tempol, have also proven useful in preventing necrosis and dysfunction after VF and cardiopulmonary resuscitation (CPR). VF was induced in rats, and after $5 \mathrm{~min}, \mathrm{CPR}$ and electrical shocks were used to induce a return to spontaneous circulation. Administering ascorbic acid or tempol at the start of CPR in a murine model of $I / R$ was correlated with reduced myocardial necrosis and lipid peroxidation, preserved mitochondrial respiratory function, and a higher and faster rate of return of spontaneous circulation, along with better survival rates at $72 \mathrm{~h}$ post-CPR (Tsai et al., 2011).

Since the endoplasmic reticulum (ER) is yet another site where redox signaling is integrated, it is a potential ROS control organelle. Selenium (Se) is essential for the function of at least 25 selenoproteins, including the antioxidant glutathione peroxidase (GPx). In patients undergoing primary coronary artery bypass grafts, preoperative reduced levels of Se, GPx, and malondialdehyde correlated with high incidence of postoperative AF (McDonald et al., 2016). Moreover, low Se concentration is inversely associated with coronary heart diseases in observational studies (Flores-Mateo et al., 2006). In animal studies, administration of selenoprotein $\mathrm{T}$ (PSELT5) prevented the increase of cardiac ROS levels and infarct size area following I/R injury (Rocca et al., 2018). In primary culture of cardiomyocytes, low concentration of Se impaired mitochondrial function and reduced expression of Kv1.2 channel (Zhang C. et al., 2017). Although no electrophysiologic consequences were provided in this study, a reduced $\mathrm{I}_{\mathrm{K} 1}$ would predict $\mathrm{AP}$ prolongation and the facilitation of EADs. Recently, it has been described that ATF6, an ER transcription factor, is responsible for inducing antioxidative stress programs, such as catalase and ROS scavenging selenoproteins, in a setting with a high level of accumulation of misfolded proteins, such as in I/R (Jin et al., 2017). Selenoproteins were also shown to recover contractility and reduce infarct size in an isolated rat heart $\mathrm{I} / \mathrm{R}$ model (Rocca et al., 2018) and treatment with dexmedetomidine, a sedative and analgesic with antioxidant properties, along with $\mathrm{Na}_{2} \mathrm{SeO}_{3}$, a Se supply for selenoproteins synthesis, was shown to confer cytoprotection against cellular damage in a cellular model of oxygen and glucose deprivation/reoxygenation by reducing apoptosis, LDH release, and ROS levels (Wang et al., 2020). However, the clinical application of Se should be taken with caution since it was demonstrated that the administration of this element in a patient population with acute myocardial infarction associated with HF did not prevent the appearance of ventricular arrhythmia (Tränkmann et al., 1999). Thus, it is possible that supplementation of selenium may be more beneficial as a preventive therapy. Nonetheless, this places the possibility of targeting the ER antioxidant resources as a mean to keep ROS levels down in a pathological setting with a high risk of arrhythmia.

Other antioxidants, such as quercetin and resveratrol, have been useful in preventing cellular damage and preserving mitochondrial functioning upon challenge with insults in vitro models, such as I/R (Lozano et al., 2019), and in reducing right-heart remodeling and dysfunction in a murine model of pulmonary arterial hypertension (Vázquez-Garza et al., 2020). Regarding arrhythmias, treatment with quercetin has proven useful in preventing platelet aggregation and arrhythmias in an I/R murine model. The protective effect is thought to be partially caused by inhibiting platelet aggregation and thus preventing microthrombi during reperfusion, which would cause heterogenous circulation when blood flow is restored, predisposing the cardiac tissue to arrhythmias (Xiao et al., 1993). A more recent study using treatment with quercetin-filled phosphatidylcholine liposomes was able to prevent peroxynitriteinduced arrhythmias in isolated murine papillary muscle and in an I/R dog model (Soloviev et al., 2002).

Resveratrol was shown to reduce lactate dehydrogenase levels, an indicator of cellular damage, as well as increase NO levels in carotid blood during $\mathrm{I} / \mathrm{R}$ arrhythmias, although there was no effect on ischemia-induced arrhythmias and mortality (Hung et al., 2000). It was also observed that cardiomyocytes from human-induced pluripotent stem cells from patients with very long-chain acyl-CoA dehydrogenase deficiency presented with a shorter APD, as well as a greater incidence of DADs and higher cytosolic $\mathrm{Ca}^{2+}$ concentration. Resveratrol treatment reportedly abolished all these changes in one of the two patients enrolled in the study, presumably by increasing the amount of defective mitochondrial proteins, which had residual activity; however, the other patient had a more severe form of the disease and no residual activity (Knottnerus et al., 2020). This decreased the accumulation of fatty acid oxidation intermediates, which seem to have caused the changes described and is a possible explanation for the higher risk of arrhythmias in patients with this genetic disease.

Another potential molecule worth mentioning is DLdithiothreitol (DTT), which restores thiol groups in oxidized proteins to their reduced state, somewhat inhibiting ROS effects. Treatment with DTT reversed $\mathrm{Ca}^{2+}$-handling disorders observed in isolated atrial cardiomyocytes after exposure to TNF- $\alpha$, such as a decreased transient amplitude, increased spark frequency and duration, and increased mitochondrial ROS production (Zuo et al., 2018), suggesting that antioxidant therapy is a promising anti-arrhythmic therapy. Finally, it has also been demonstrated that bongkrekic acid, an adenine nucleotide translocase (ANT) inhibitor, has potential as an antiarrhythmic drug. In a murine model of fatty acid accumulation, fatty acids caused a partial inhibition of ANT, which was associated with 
increased ROS production, decreased $\Delta \Psi \mathrm{m}$, RyR2 oxidation, decreased $\mathrm{Ca}^{2+}$ transient amplitude, increased $\mathrm{Ca}^{2+}$ sparks and $\mathrm{Ca}^{2+}$ wave incidence, and an increased propensity to develop non-sustained ventricular tachycardia. Adding bongkrekic acid or $\mathrm{N}$-acetylcysteine decreased ROS production, RyR2 oxidation, and $\mathrm{Ca}^{2+}$ spark frequency, as well as preserved $\Delta \Psi \mathrm{m}$ and the $\mathrm{Ca}^{2+}$ transient amplitude and prevented the development of non-sustained ventricular tachycardia (Roussel et al., 2015).

It was previously thought that antioxidants like quercetin and resveratrol, due to their low bioavailability and nonspecificity to any tissue, would make them poor candidates for new therapies (Formica and Regelson, 1995). This is backed by the fact that only limited benefits have been found when using such therapies (Askari et al., 2012; Wilson et al., 2016; Vázquez-Garza et al., 2020). Nonetheless, similar to the gene therapy described previously, the development of nanomaterials capable of packaging the components, protecting them from degradation, and directing them to a specific tissue places these often-dismissed compounds back on the table. This new way of delivering compounds has opened a wide spectrum of possible components that were disregarded in the past due to having low bioavailability or a small therapeutic window or because they were unable to pass through the cellular membrane. Furthermore, nanomaterials make it possible for two or more different compounds to be placed in the same particle, increasing the number of possibilities exponentially. A more in-depth review of nanoparticles is available (Lozano et al., 2018). Even drugs that are being used today may be packaged to provide the same or a better outcome while reducing adverse effects. It is only a matter of time before these compounds demonstrate their real potential in preserving mitochondrial integrity in a pathologic setting, such as arrhythmias. Previous clinical trials targeting ROS scavenging as an antiarrhythmic therapy have had mixed results (El-Hamamsy et al., 2007; Hicks et al., 2007; Negi et al., 2011; Rodrigo et al., 2013; Martínez-González et al., 2014; Stanger et al., 2014), some of which may be debated to be due to other effects, not only ROS scavenging (Martínez-González et al., 2014). Furthermore, a meta-analysis of clinical studies with commonly used antioxidants concluded that the studies with positive results were flawed by the small sample size and the lack of evidence of a real antioxidant effect in these patients (Violi et al., 2014). Nonetheless, there have been no clinical trials targeting mitochondrial ROS specifically, and thus the possibility of this being a new therapeutic target should not be dismissed.

\section{Specific Targeting of Mitochondrial ROS Production}

MitoTEMPO, a mitochondria-specific molecule, is composed of a combination of the antioxidant piperidine nitroxide tempo and the lipophilic cation triphenylphosphonium. The lipophilic cation section of the molecule enables it to pass through bilipid layers and accumulate predominantly where there is an accumulation of negative charges, which means that it is drawn toward $\Delta \Psi \mathrm{m}$. Mitochondrial concentrations of MitoTEMPO are several 100-fold when compared to cytosol. This makes it a suitable candidate to prevent excessive mitochondrial ROS accumulation and has already been tested in a murine model of diabetic cardiomyopathy. Daily dosing reduced superoxide generation in cardiomyocytes, reduced mitochondrial ROS generation, decreased apoptosis, and reduced myocardial hypertrophy, which were increased in the diabetic group without MitoTEMPO. The treatment group was also found to have a preserved ejection fraction and fractional shortening when measured by echocardiography, both of which were reduced in the diabetic group without MitoTEMPO (Ni et al., 2016). Similarly, in isolated rabbit cardiomyocytes, ISO exposure was associated with increased $\mathrm{Ca}^{2+}$ leakage, $\mathrm{Ca}^{2+}$ waves, and ROS production, as evidenced by fewer free thiols in the RyR2. These effects were partially prevented using MitoTEMPO or other ROS scavengers (Bovo et al., 2012) and were corroborated in a murine model of CPVT (Hamilton et al., 2020). MitoTEMPO has also been described as being able to prevent sudden cardiac death and ventricular arrhythmias in an HF guinea pig model, as well as prevent remodeling (Dey et al., 2018). Other mitochondriaspecific molecules have also produced similar results. In an I/R model, peptides SS-31 and SS-20 reduced infarct size and severity, as well as the duration of reperfusion arrhythmias and lipid peroxidation, most likely by scavenging and reducing ROS production (Cho et al., 2007). Observations of human ventricular myocytes from patients with end-stage HF further support this idea. In this condition, it has been shown that RyR2s become uncoupled from nearby T-tubules and prone to spontaneous activation. Treatment with mitochondrial ROS scavengers reduces the receptor's hyperactivity and spontaneous $\mathrm{Ca}^{2+}$ wave formation under adrenergic stimulation (Dries et al., 2018). These compounds are, therefore, another viable treatment option to reduce mitochondrial ROS accumulation and subsequent mitochondrial dysfunction.

\section{Modulation of mPTP Opening}

Despite its elusive composition, the mPTP is strongly regulated by cyclophilin D (CypD), the VDAC, ANT, the phosphate carrier, ATP synthase, and the translocator protein (TSPO), formerly known as the peripheral benzodiazepine receptor (Alves-Figueiredo et al., 2021). A causative relationship between ROS-induced mPTP opening and $\Delta \Psi_{\mathrm{m}}$ depolarization has also been demonstrated in numerous studies. In particular, there is some evidence that the mPTP may also be regulated by TSPO through interaction with the other components (Zulian et al., 2011). Using TSPO ligands has been shown to inhibit $\Delta \Psi_{\mathrm{m}}$ depolarization after excessive ROS exposure (Leducq et al., 2003). TSPO became known as a potential therapeutic target when it was shown to activate a channel in the IMM, the inner membrane anion channel (IMAC). This channel is described as being able to depolarize $\Delta \Psi_{\mathrm{m}}$ in an mPTP-independent way, causing oscillations in $\Delta \Psi_{\mathrm{m}}$ and intermittent sarcKATP channel activation (Aon et al., 2003). These oscillations cause variation in the APD, which promotes a reentry pathway to the electrical stimuli. Inhibiting TSPO, therefore, has the potential to cover two different pathways to mitochondrial dysfunction, IMAC-mediated and mPTP-mediated (Gambardella et al., 2017), making this protein a potential therapeutic target. Although studies have shown that TSPO ligands can reduce ROS levels and abolish metabolic and electrophysiological changes, they have yet to be tested in a clinical setting (Akar et al., 2005). Protection 
against mitochondrial depolarization has been observed to translate into reduced APD shortening and inexcitability in a dose-dependent manner. However, the use of FGIN-1-27, an IMAC activator, promoted faster shortening of the APD and resulted in an earlier loss of conduction during reperfusion (Akar et al., 2005). Hearts treated with an IMAC activator prior to ischemia are more responsive to it. High-resolution optical mapping demonstrated small areas of conduction blockage during early ischemia that persisted throughout the whole recording, making it more suitable for reentry, which caused VF. IMAC inhibition stabilized $\Delta \Psi \mathrm{m}$ in vitro, suppressed reperfusion arrhythmias, and promoted faster AP recovery (Akar et al., 2005). The protective effect on AP recovery was also demonstrated in a rabbit model of I/R (Brown et al., 2008). Notably, the antiarrhythmic effect afforded by TSPO ligands was not evident in hearts treated with the MPTP desensitizer CsA. This finding indicates that IMAC and MPTP opening are primary mitochondrial mediators of acute postischemic arrhythmias. Finally, mPTP opening, which can be viewed as an irreversible loss of mitochondrial function, leads to cell death. Over time, cardiomyocytes are replaced with fibrotic tissue, which generates a physical barrier that prevents proper stimulation of the myocardium in an orderly manner. This pathological remodeling creates possible paths for re-entry arrhythmias.

As mentioned previously, MPTP opening is the process by which mitochondrial membrane integrity is permanently lost, and content from the mitochondrial matrix flows toward the cytosol. Thus, the mitochondria are no longer able to maintain their $\Delta \Psi \mathrm{m}, \mathrm{ATP}$ is unable to be synthesized, and there is a subsequent loss of ionic balance. Between the leaked components that are translocated to the cytosol, cytochrome C, which normally takes part in the ETC, starts a signaling cascade for cell apoptosis. Cardiomyocyte death may be the most challenging arrhythmogenic process to deal with, as cardiomyocytes do not regenerate and are replaced with fibrotic tissue. This fibrotic tissue not only reduces the myocardium's pool of available contractile units to create effective blood pumping but also creates zones in which electrical signals do not travel at the proper speed, paving the way for disorganized contraction. CsA, a well-known immunosuppressor, was found to be a potent mPTP inhibitor many years ago (Crompton et al., 1988). Later, it was found that the compound's inhibitory effect on the MPTP is achieved by inhibiting CypD, a component required for mPTP opening (Davidson and Halestrap, 1990; Griffiths and Halestrap, 1991). It was discovered that inhibiting MPTP with CsA reduces I/R-induced arrhythmias by preserving cardiac tissue from suffering edema and reducing cardiomyocyte death, presumably by protecting mitochondria from $\mathrm{Ca}^{2+}$-induced damage (Arteaga et al., 1992). mPTP inhibition was then corroborated as a potential strategy when another inhibitor, octylguanidine, was observed to protect against arrhythmias and edema caused by $\mathrm{I} / \mathrm{R}$, as well as maintain blood pressure and mitochondrial function (Parra et al., 2005). The effect was later confirmed in a hyperthyroid murine model undergoing I/R. Octylguanidine protects against arrhythmias by maintaining mitochondrial functioning and cardiac hemodynamic parameters and reducing inflammatory cytokines (Pavón et al., 2009). Another mPTP inhibitor, citicoline, was also useful in preventing arrhythmia and blood pressure drop during I/R. Mitochondria maintained adequate $\Delta \Psi \mathrm{m}$, high respiratory control, cis-aconitase enzyme activity, and mDNA integrity (Hernández-Esquivel et al., 2014). It was then shown that MPTP inhibition by CsA administration was related to reduced infarct size in a murine model of $\mathrm{I} / \mathrm{R}$ (Xie and $\mathrm{Yu}, 2007)$. CsA was also shown to reduce hypertrophy and arrhythmia susceptibility in a canine model of chronic atrioventricular blockage. CsA administration was not associated with reducing the electrical remodeling observed in the model, as indicated by a prolonged effective refractory period similar to the no-treatment group, although it did reduce the thickness of free and septal ventricular walls. Nonetheless, the CsA group showed no susceptibility to drug-induced polymorphic ventricular tachycardia (Schreiner et al., 2004). This led to the conclusion that electrical remodeling is only partially responsible for the susceptibility to arrhythmias seen in the model and that hypertrophy is also a necessary substrate for arrhythmogenesis. However, more recent studies found no protective effect when using CsA and measuring $\mathrm{MPTP}$ opening in vivo in a VF murine model. There were no differences in troponin I, cytochrome c, or $\mathrm{NAD}^{+}$levels when compared to the group treated with just the vehicle, as well as in hemodynamic and left ventricular (LV) function parameters (Ayoub et al., 2017). Although it could be argued that CsA was administered at half the dosing concentration used in previous studies and was administered for only $5 \mathrm{~min}$ before inducing VF or before starting the resuscitation protocol. Similar results were found when comparing $4^{\prime}$ chlorodiazepam (4-ClDzp), a ligand for TSPO, to CsA in a rabbit I/R model. CsA offered no protection against arrhythmias, in contrast to 4-ClDzp, which preserved normal cardiac functioning when given as an infusion throughout the I/R or as a single bolus before reperfusion (Brown et al., 2008). Another study utilized guinea pig hearts in an I/R protocol. 4-ClDzp preserved $\Delta \Psi \mathrm{m}$, reduced AP shortening, and prevented reperfusion arrhythmias. Recovery was remarkably higher than in the group treated with CsA (Akar et al., 2005). This evidence raises the possibility of yet another potential target for new anti-arrhythmic therapies, and some authors have even proposed that the mediator of postischemic arrhythmias is the IMAC, which can be regulated via TSPO, rather than the MPTP (Motloch et al., 2015). However, a CypD KO murine model proved that the MPTP does, indeed, play a role in arrhythmogenesis. At the cellular level, cardiomyocytes from CypD KO mice were much less likely to undergo mPTP opening upon the addition of FCCP. Likewise, the incidence of $\mathrm{Ca}^{2+}$ waves and $\mathrm{Ca}^{2+}$ alternans was much lower than in their WT counterparts. Similar results were also obtained when CsA was added to WT cardiomyocytes. At the ex vivo heart level, ECGs showed ST-T wave alternans in the WT group, but the alternans were absent in the CypD KO group and only observed in one heart in the CsA group. Arrhythmia scores were consistently much lower upon FCCP addition or I/R (Gordan et al., 2016). Using the same CypD KO model, arrhythmia susceptibility was tested under an iron overload protocol. Again, WT cardiomyocytes presented with increased ROS production, diminished $\Delta \Psi \mathrm{m}$, and frequent $\mathrm{Ca}^{2+}$ waves, while ex vivo 
hearts presented with arrhythmias upon stimulation. Although the CypD KO group presented with similar ROS production and decreased $\Delta \Psi \mathrm{m}$, its $\mathrm{Ca}^{2+}$ waves were significantly less frequent, and arrhythmia scores were lower. Similar results were found when the WT group was treated with CsA (Gordan et al., 2020). Although there may be contradictory evidence, CsA is still thought to be a molecule with therapeutic potential.

These findings have led to the use of CsA as a positive control for mitochondrial protection against mPTP, with which other treatments can be compared. However, when CsA was used as a treatment for out-of-hospital cardiac arrest in the CYRUS trial, there was no difference in organ failure, survival at hospital admission, survival at $24 \mathrm{~h}$, survival at hospital discharge, or favorable neurologic outcomes when compared to the control group (Argaud et al., 2016). This finding led to the reasoning that in clinical practice, patients who are susceptible to cardiac arrest or have a baseline cardiopathy have already undergone adaptations in their cardiac tissue that make them less susceptible to MPTP protection with CsA, unlike murine models of ischemiareperfusion that use healthy and young animals in both groups. After integrating the information presented here, it is possible that MPTP inhibition delays irreversible mitochondrial damage, giving the cardiomyocyte time to fix the underlying cause. It may be helpful in a young, healthy animal model in which the insult is temporal and where the cardiac tissue can deal with the ROS generated after reperfusion, given the extra time CsA provides before mPTP opening. However, in the context of a patient with severe atherosclerosis or HF, which is more commonly the case in patients with a high risk of arrhythmias, there is already increased ROS production and $\mathrm{Ca}^{2+}$ overload, which decreased the cell's resources considerably before ischemia. Delaying mPTP opening would have little effect in preserving cardiac tissue since the cardiomyocyte is unable to re-establish homeostatic conditions, even if given more time with CsA. Nonetheless, there is still the possibility that CsA, used in combination with another therapy, could synergize by delaying MPTP opening, and other therapies could take effect in cardiomyocytes, which would not have had enough time for recovery otherwise.

\section{Regulation of Mitochondrial Quality Control}

Excessive mitochondrial fission has been observed to promote cell death in I/R models. Mdivi-1, a fission inhibitor that has been shown to prevent apoptosis, and M1, a cell-permeable phenylhydrazone fusion promoter, were administered in an I/R murine model (Maneechote et al., 2018, 2019). Both treatments significantly reduced arrhythmias after reperfusion when given prior to ischemia, as well as reduced the decline in hemodynamic parameters. Preventing excessive fission with the subsequent reduction in $\Delta \Psi \mathrm{m}$ and ATP synthesis and increasing ROS generation is yet another potential therapeutic strategy to prevent arrhythmia generation in high-risk patients. As stated previously, a pressure-overload HF murine model was shown to have a blockade in the cell's mitophagic function, leading to damaged mitochondria accumulation. When given the $\mathrm{mCU}$ inhibitor $\mathrm{RuR}$ or $\mathrm{Ru}_{360}$, the mitophagic profile improved to reveal no blockade, along with improvement in cardiac function, reduction in ventricular asynchrony, and preserved mitochondrial integrity (Yu et al., 2018). This finding indicates that some therapies, such as mCU modulation, have effects at different levels and signaling pathways. Further research is needed to elucidate the mechanisms by which this happens to better understand the effects that such strategies have at the mitochondrial and cellular levels.

\section{Maintaining Energetic Balance With High-Energy Phosphate Analogs}

A central point of $\mathrm{Ca}^{2+}$ dysregulation is the energetic deficiency that ensues given the lower ATP production by dysfunctional mitochondria, accompanied by higher energetic demand from a less efficient $\mathrm{Ca}^{2+}$ removal system. Thus, replenishing the energetic reserve with exogenous molecules is another possible approach. In this regard, cyclocreatine, a creatine analog that is permeable to membranes, has been used mainly as a therapy for I/R injury. Prior treatment with cyclocreatine in the diet has been shown to preserve ATP levels longer during ischemia in chicken hearts (Turner and Walker, 1985) when measured by conventional techniques, as well as in rat hearts when measured via nuclear magnetic resonance imaging (Osbakken et al., 1992). Cyclocreatine has also been demonstrated to have anti-inflammatory effects in isolated heart (Elgebaly et al., 1990) and in vivo (Elgebaly et al., 1993) models of ischemia. Recovery of cardiac function after cold storage has also been improved when hearts were previously treated with cyclocreatine phosphate (Elgebaly et al., 1994), as well as in models of cardiopulmonary bypass (Houser et al., 1995). Previous studies have only been conducted in the context of ischemia and in clinical trials using other high-energy phosphate analogs without favorable results in HF or myocardial infarction patients (Horjus et al., 2011). However, it may still be worthwhile to research the effects of compounds like cyclocreatine in arrhythmia models, as it has been stated that the failure of these substances to preserve ATP levels and, thus, cardiac function may be due to kinetic differences in their ability to become a substrate for creatine kinase and their phosphoryl group transfer potential. Although monotherapy with high-energy phosphate analogs may not be effective in preventing arrhythmias, it can still potentiate the effects of other therapies by increasing and preserving the heart energy pool.

\section{MitoK $_{\text {ATP }}$ Channel Modulators}

MitoK $_{\text {ATP }}$ channels are structures within the IMM that, upon opening, partially dissipate $\Delta \Psi \mathrm{m}$. This partial depolarization diminishes the driving force for $\mathrm{Ca}^{2+}$ entry and thus prevents $\mathrm{Ca}^{2+}$ overload and generates ROS, which have been associated with the protection conferred by ischemic preconditioning (Pain et al., 2000). Agonists of this channel have been assessed as a possible therapeutic approach. 4-carboxy phenyl-isothiocyanate (4CPI), a hydrogen sulfide (H2S) pro-drug and mitoK $\mathrm{KTP}_{\mathrm{AT}}$ agonist, reduced tissue injury and ROS production, prevented arrhythmias, and improved recovery upon reperfusion in murine models of I/R; these effects were lost when 5-hydroxydecanoic acid, a mitoK $K_{\text {ATP }}$ blocker, was added (Testai et al., 2016). 
Compound $\mathrm{A}$, another mito $\mathrm{K}_{\mathrm{ATP}}$ agonist, has also been proven to reduce reperfusion arrhythmias in $I / R$, with effects even more pronounced than diazoxide, a more commonly used mitoK $K_{\text {ATP }}$ agonist. Unfortunately, these effects were not more effective when used in combination with a sarcolemmal $\mathrm{K}_{\mathrm{ATP}}$ blocker (Gonca et al., 2016). Even sevoflurane, a commonly used anesthetic, has been demonstrated to have protective effects dependent on mitoK $\mathrm{K}_{\mathrm{ATP}}$ opening. Preconditioning isolated rat hearts with sevoflurane was demonstrated to have protective effects upon I/R, as evidenced by smaller infarct size, reduced cardiac troponin I levels, upregulated PKC, and downregulated caspase 8 . This protection was lost when hearts were pretreated with 5-hydroxydecanoic acid, suggesting the role of mitoK $\mathrm{K}_{\mathrm{ATP}}$ in the protective effects of sevoflurane (Wang et al., 2015). While these results seem promising, more studies are needed, mainly in other models susceptible to generating arrhythmias, to obtain more solid evidence that mitoK $_{\mathrm{ATP}}$ is a potential antiarrhythmic therapy.

In the last few years, there has been considerable advancement in understanding the molecular mechanisms of cardiac disease. This has laid a path to the development of better therapeutic strategies that were first ignored or disregarded as potentially ineffective. It has also led to the discontinuation of practices that were once thought to be helpful but then proved to be either neutral or damaging. It may be time to expand our viewpoint and explore the crosstalk that takes place between organelles and seems to orchestrate $\mathrm{Ca}^{2+}$ homeostasis rather than focus on a single cellular component for a new therapy to prevent sudden cardiac death. This may be the right way to prevent arrhythmogenesis, by tackling the central mechanism that leads to different cellular malfunctions.

\section{Mitochondrial-SR Interaction CAMKII Modulation to Prevent SR $\mathrm{Ca}^{2+}$ Leaks Into Mitochondria}

$\mathrm{Ca}^{2+} /$ calmodulin-dependent protein kinase II (CAMKII) is an enzyme normally activated by $\mathrm{Ca}^{2+}$-calmodulin and phosphorylates a wide range of proteins, including RyR2, PLB, and LTCCs. Increased CAMKII activity has been observed in pathological processes, such as cardiac hypertrophy and HF (Kirchhefer et al., 1999; Zhang et al., 2003). Whether a higher phosphorylated state of the RyR2 leads to a subsequently higher opening probability in diastolic $\mathrm{Ca}^{2+}$ concentrations, the generation of $\mathrm{Ca}^{2+}$ sparks, lower SR Ca ${ }^{2+}$ content, and a negative force-frequency relationship (Ai et al., 2005; MacDonnell et al., 2008; Kushnir et al., 2010) remains controversial. Unsurprisingly, CAMKII has been a potential target in the prevention of hypertrophy, arrhythmias, and HF. Modulating its activity has led to interesting results regarding cardiac tissue protection. KN-93, the most well-known CAMKII inhibitor, has been shown to prevent AF in mice with a gain-of-function mutation in the RyR2 after fast atrial pacing (Chelu et al., 2009). AIP, another CAMKII inhibitor, has also been shown to prevent afterdepolarization events in isolated cardiomyocytes in a murine model overexpressing CaMKII $\delta_{C}$. Additionally, KN-93 administration in vivo prevented ISO-induced arrhythmias (Sag et al., 2009). In a murine model of diabetic hyperglycemia, KN93 administration reduced premature ventricular contractions, as well as $\mathrm{Ca}^{2+}$ sparks, and was further supported with similar findings from diabetic failing heart donors (Erickson et al., 2013). This treatment strategy has also proved to be effective in a murine model expressing constitutively active $\mathrm{Ca}^{2+}$-dependent phosphatase calcineurin (CAN), in which KN-93 administration reduced arrhythmia susceptibility, as well as improved ventricular cardiomyocyte contractile functioning (Khoo et al., 2006). Similarly, an impaired glucose tolerance murine model was found to have arrhythmias and arrhythmogenic events related to hyperphosphorylation of $\mathrm{Ca}^{2+}$ handling proteins in isolated ventricular cardiomyocytes, which were prevented when a transgenic model that expressed AIP was submitted to the same impaired glucose tolerance protocol (Federico et al., 2019). Furthermore, CaMKII also regulates mitochondrial functioning by controlling fatty acid oxidation through palmitoyl-CoA transferase I (Sharma et al., 2010) and $\mathrm{Ca}^{2+}$-dependent dehydrogenases via the mCU (Joiner et al., 2012); however, some studies do not support this conclusion (Nickel et al., 2020). CaMKII might enhance mCU activity by phosphorylating the mCU's N-terminals and concomitantly lead to mPTP opening by mitochondrial $\mathrm{Ca}^{2+}$ overload and, ultimately, cell death upon I/R injury or dilated cardiomyopathy (Joiner et al., 2012). Chronic CaMKII activation has also been shown to be involved in mitochondrial gene reprogramming, leading to mitochondrial dysfunction and increased oxidative stress in cardiac hypertrophy (Joiner and Koval, 2014). CaMKII also mediates Drp1 phosphorylation, which has been shown to increase the frequency of $\mathrm{mPTP}$ opening upon chronic $\beta$-adrenergic stimulation (Xu et al., 2016). Although the use of a CaMKII inhibitor has not been demonstrated in mitochondrial physiology, KN-93 administration has also been used in a murine model of CPVT. Arrhythmias were abolished when treated with $\mathrm{KN}-93$, and the spark frequency was reduced in isolated cardiomyocytes when treated with ISO (Liu et al., 2011). These findings are further supported by cardiomyocytes derived from induced pluripotent stem cells of CPVT patients presenting with similar findings. All this evidence suggests that CAMKII activation plays a major role not only in arrhythmogenesis but also in other cardiac pathologies, such as HF progression. Further studies regarding the development of a more specific component with a much higher affinity could bring us closer to the development of a new therapeutic regime.

\section{RyR2 Stabilizers to Reduce Mitochondrial $\mathrm{Ca}^{2+}$ Overload}

Dantrolene is a muscle relaxant known widely for its use in treating malignant hyperthermia. This condition arises when a patient with a mutation in the RyR2 is exposed to inhaled halogenated anesthetics. This mutation makes the RyR2 susceptible to massive $\mathrm{Ca}^{2+}$ release, which is associated with muscle breakdown, elevated serum creatinine kinase, hypotension, hyperthermia, tachycardia, and intraoperative death. Its mechanism of action was unknown until a few years ago, when it was found to bind the RyR2 and stabilize domains within the receptor (Paul-Pletzer et al., 2002; 
Kobayashi et al., 2005). Its mechanism of action seems suitable for preventing stochastic $\mathrm{Ca}^{2+}$ release during diastole and has been studied in various scenarios. It has shown therapeutic potential in a murine model of CPVT, in which mice were subjected to a knock-in procedure of a known mutation that causes the pathology. Transgenic mice presented with ventricular tachycardia when stimulated with epinephrine or by running on a treadmill. Pretreatment with dantrolene for 7-10 days inhibited VT. In isolated cardiomyocytes, the spark frequency was also diminished (Kobayashi et al., 2010). In an ex vivo model of long-QT syndrome, rabbit hearts were infused with erythromycin or veratridine to mimic the condition. When the potassium concentration was lowered, the hearts presented with early EADs and polymorphic ventricular tachycardia. Infusion with dantrolene resulted in a significant reduction of EADs and polymorphic VT (Frommeyer et al., 2018). Dantrolene also regulates mitochondrial-SR interconnection in cardiomyocytes from a model of HF with increased stochastic RyR2 activity. The author identified that spontaneous $\mathrm{Ca}^{2+}$ waves, which can subsequently propagate and trigger organ-wide arrhythmia, were increased in the presence of the mCU agonist (Hamilton et al., 2018). Under these conditions, kaempferoltreated ventricular myocytes showed a significant increase in the percentage of cells presenting with spontaneous $\mathrm{Ca}^{2+}$ waves. Interestingly, stabilization of the RyR2 with dantrolene reduced the proarrhythmic effects of kaempferol on $\mathrm{Ca}^{2+}$ handling (Hamilton et al., 2018). In this context, some authors (Beutner et al., 2001) have identified a RyR2-like protein in the IMM. This elusive $\mathrm{Ca}^{2+}$ transporter demonstrates that it might be possible to modulate dantrolene-sensitive mitochondrial functions, such as $\mathrm{Ca}^{2+}$-dependent $\mathrm{O}_{2}$ consumption, $\mathrm{Ca}^{2+}$-dependent $\mathrm{MPTP}$ and swelling, and cytochrome c release (Beutner et al., 2001), with a direct effect on mitochondrial RyR2.

Dantrolene has also been used to treat AF in a sheep model of atrial infarction. Left atrial myocardial infarction was induced by ligating the atrial branch of the left anterior descending coronary artery. After the procedure, numerous episodes of AF appeared. It was noted that these episodes were produced by spontaneous focal discharges occurring in the zone between the infarcted and normal tissue and were inducible with ISO. Dantrolene administration after the procedure prevented the focal discharges and AF, presumably by maintaining a proper RyR2 response to calmodulin (Avula et al., 2018). It has also been compared to amiodarone in VF treatment using a pig model of cardiac arrest. Under anesthesia, VF was induced by an electrode in the right ventricle and left untreated for $8 \mathrm{~min}$. Afterward, chest compressions and ventilation were started, along with the administration of either amiodarone, dantrolene, or saline. After 4 min of resuscitation, defibrillation was attempted. The rates of return to spontaneous circulation and hemodynamic stability were not statistically different in the dantrolene and amiodarone groups (Wiesmann et al., 2017). This finding implies that dantrolene is as effective as other anti-arrhythmic drugs currently in use. In a more clinical setting, dantrolene has shown efficacy in reducing spark frequency, diastolic SR $\mathrm{Ca}^{2+}$ leakage, $\mathrm{Ca}^{2+}$ waves, and spontaneous $\mathrm{Ca}^{2+}$ transients in isolated human cardiomyocytes from patients with $\mathrm{AF}$ or end-stage $\mathrm{HF}$
(Hartmann et al., 2017). Similarly, a small group of patients with CPVT underwent an exercise stress test, and ECG was recorded before and after dantrolene administration. Premature ventricular complexes were reduced in patients enrolled in the study. Similarly, cardiomyocytes derived from induced pluripotent stem cells from fibroblasts from these patients were stimulated with epinephrine, and $\mathrm{Ca}^{2+}$ transients were recorded. Dantrolene, again, abolished most of the abnormalities registered in patients (Penttinen et al., 2015). Notably, some patients who responded to treatment had mutations in the $\mathrm{N}$-terminal or central cytosolic region of the RyR2 protein, while the nonresponders had their mutations in or near the transmembrane domain. This brings attention to the fact that, at least in the case of CPVT, the baseline pathology needs to be checked to ensure pharmaceutical effectiveness.

However, dissociation of this protein with the RyR2 macromolecular complex has been described in a variety of cardiac pathologies, such as HF, hereditary forms of exerciseinduced arrhythmias, and sudden cardiac death. Dissociation of this protein causes the RyR2 to become more sensitized to $\mathrm{Ca}^{2+}$-dependent activation, increasing diastolic $\mathrm{Ca}^{2+}$ leakage, impairing $\mathrm{Ca}^{2+}$ cycling, and decreasing contractility (Lehnart et al., 2004b). Increasing the affinity of calstabin2 to the RyR2 macromolecular complex with the 1,4-benzothiazepine derivative molecule JTV519 or S107 has been useful in preventing arrhythmias and sudden cardiac death. In one of the first experiments, JTV519 was found to correct abnormal RyR2 channel gating in dogs with induced HF (Kohno et al., 2003). In another study, calstabin $2^{ \pm}$mice presented with ventricular tachycardia and sudden cardiac death when put under strenuous exercise. However, these events were prevented entirely when treated with JTV519 (Wehrens et al., 2004). Treatment with JTV519 was associated with a decreased opening probability of individual RyR2 channels when subjected to diastolic $\mathrm{Ca}^{2+}$ concentrations compared to the untreated group, which had abnormally high opening probabilities. Calstabin $2^{-/-}$mice, however, exhibited no protection from JTV519, demonstrating that calstabin 2 must be present for JTV519 to prevent stochastic RyR2 channel opening. This molecule has also been shown to stabilize RyR2 opening in the context of mutations found in CPVT patients. Treatment with JTV519 was found to increase the association between calstabin2 and the RyR2 and normalize the channel's functioning (Lehnart et al., 2004a). This was recently demonstrated using 3D-engineered heart tissue transfected to express channelrhodopsin-2, an ion channel activated by light. Under a chronic optical tachypacing protocol, the tissue remodeling showed a propensity for tachycardic episodes when submitted to a burst pacing protocol. This propensity was abolished when JTV519 was administered (Lemme et al., 2019). In addition, in guinea-pig ventricular muscles subjected to no-flow ischemia followed by reperfusion, JTV519 administration significantly improved post-ischemic contractile recovery. However, the potential benefit of JTV519 administration in models of $\mathrm{I} / \mathrm{R}$ injury was blocked with 5 -HD, thereby suggesting that the activation of mitochondrial $\mathrm{K}_{\text {ATP }}$ channels mediates the cardioprotective effects of JTV519 (Ito et al., 2000). 
Given the non-specific effects that JTV519 could have on other ion channels, a new molecule, S107, was initially developed as a treatment to increase skeletal muscle exercise capacity (Bellinger et al., 2008). However, this new molecule prevented arrhythmias and seizures in mice harboring a CPVT mutation in the RyR2 (Lehnart et al., 2008). The protective effect was also observed in a Duchenne muscular dystrophy (DMD) murine model. DMD mice were found to have structural remodeling in their cardiac tissue. This remodeling predisposed the model to ventricular arrhythmias, which were prevented completely with S107 administration (Fauconnier et al., 2010). S107 was also able to suppress AF in different murine models with knock-in mutations known to cause CPVT in humans (Shan et al., 2012). In another study using cardiomyocytes derived from induced pluripotent stem cells from a human patient with CPVT, preincubation with S107 prior to ISO exposure dramatically reduced the percentage of cells that developed DADs (Sasaki et al., 2016). Finally, a new optogenetic animal model using nematodes with mutations in proteins analogous to mutations in the RyR2 or calsequestrin in CPVT patients displayed similar muscular dysfunction findings upon a stress challenge with a faster pacing rate; muscular dysfunction was prevented with the use of S107 (Fischer et al., 2017).

\section{SERCA Modulation to Improve ECEC}

Numerous studies have shown that SERCA's functionality is reduced in common cardiac pathologies and conditions prone to developing arrhythmias. Such is the case of AF, in which reduced SERCA expression has been described in the peripheral blood cells of patients with AF, and these levels of expression can be used to predict the clinical response to treatment, such as epicardial thoracoscopic PV isolation (Sardu et al., 2020). Unsurprisingly, increasing SERCA's capacity to pump $\mathrm{Ca}^{2+}$ into the SR to increase SR $\mathrm{Ca}^{2+}$ content, which, in turn, increases the amount of $\mathrm{Ca}^{2+}$ released during CICR and translates into generating a greater contractile force, along with a lower probability of stochastic RyR2 opening, by decreasing cytosolic $\mathrm{Ca}^{2+}$ concentrations during diastole. This is one of the most studied approaches to treating cardiac dysfunction and has been assessed from different viewpoints. A small group of patients with advanced HF received an intracoronary infusion of an adeno-associated viral vector (AAV) containing genetic material that coded for SER-CA2a. These patients presented with improvements in symptomatic, functional, biomarker, and LV functional parameters, along with no significant adverse effects in other organs (Jaski et al., 2009). Notably, two patients who did not experience clinical improvement despite the therapy already had circulating antibodies against the viral vector before transfusion. In a phase 2a trial, 39 patients received an intracoronary infusion of the AAV. The same parameters were assessed. Again, the treatment group presented with improvement, an increased time from therapy to the occurrence of clinical events related to HF, and a decreased frequency of cardiovascular events in the following 12 months (Jessup et al., 2011). However, when the phase $2 \mathrm{~b}$ trial was conducted, unfortunately, after a median follow-up of 17.5 months, the treatment group showed no improvement in time to recurrent events or mortality when compared to placebo (Greenberg et al., 2016). The reason these results were so different from previous studies remains unclear. It is hypothesized that the previous studies' results were affected by chance, considering the small number of patients, or the formulation used in the CUPID 2 trial was not effective enough. Although the outcome was unexpected, it at least proved gene therapy is a viable option, as there were no major adverse effects when compared to placebo. Furthermore, the trial provides insight into what needs further development before a second attempt to implement gene therapy is performed.

Another strategy used to enhance SERCA's $\mathrm{Ca}^{2+}$ pumping capacity is to functionally reduce its inhibitor protein, PLB. This strategy has been studied with a PLB KO murine model. This model presented with a similar magnitude in the LTCC current when compared to wild-type, although with a faster decay. Regarding differences, a larger AP that decayed faster had greater SR $\mathrm{Ca}^{2+}$ content, better ECC (measured as $\Delta$ cytosolic $\mathrm{Ca}^{2+}$ concentration/LTCC current), and more frequent and greater $\mathrm{Ca}^{2+}$ sparks were noted, although the decay times were similar (Santana et al., 1997). This model also presented with better diastolic functioning in Doppler and color M-mode echocardiography (Schmidt et al., 2002). Furthermore, combining a SERCA overexpression model with a PLB KO model resulted in an even more enhanced cardiac state. Mice with both genetic modifications presented with higher maximal rates of contraction and relaxation and lower $\mathrm{Ca}^{2+}$ transient decay times when compared to groups with either single mutation. No histological or pathological changes were found in the double transgenic model (Zhao et al., 2003). This demonstrates the synergy achieved when enhancing SERCA's functionality from two different approaches, making it possible to simultaneously address SERCA's dysfunction with different treatments. Interestingly, these transgenic mice exhibited increased oxygen consumption to meet the demand for increased ATP consumption. Higher levels of mitochondrial oxygen consumption were associated with increased $\mathrm{Ca}^{2+}$ dependent pyruvate dehydrogenase activity. These findings suggest that the ablation of PLB requires metabolic adaptations to establish proper ECEC (Chu et al., 1996).

Concerns about enhancing SERCA in a dysfunctional setting have been expressed. Because the RyR2 is commonly dysfunctional in most settings where SERCA is less efficient in removing diastolic $\mathrm{Ca}^{2+}$, it is implied that increasing SR $\mathrm{Ca}^{2+}$ without addressing the increased opening probability of the RyR2 could result in an enhanced spark frequency and higher $\mathrm{Ca}^{2+}$ wave propagation, caused by RyR2 opening due to increased luminal $\mathrm{Ca}^{2+}$ sensibility, with subsequent arrhythmia development. Nonetheless, studies involving the enhancement of SERCA's functioning in such settings, including HF models and patients with advanced HF, have not shown such adverse effects. This may be due to SERCA's enhanced efficiency being more beneficial by reducing diastolic $\mathrm{Ca}^{2+}$, as it seems that the RyR2 still needs cytosolic $\mathrm{Ca}^{2+}$ for stochastic opening, even under conditions of increased sensitivity to luminal $\mathrm{Ca}^{2+}$ content, since reaching a certain $\mathrm{SR} \mathrm{Ca}{ }^{2+}$ threshold seems insufficient for diastolic RyR2 opening (Belevych et al., 2012). 


\section{FINAL REMARKS}

The SR and mitochondria engage in constant, intimate communication to properly respond to workload and metabolic needs. However, their interconnectedness leaves both organelles vulnerable to malfunction if one should have its safety mechanisms overrun. In this case, conditions that favor $\mathrm{Ca}^{2+}$ overload, such as chronic adrenergic stimulation, could elevate the diastolic mitochondrial $\mathrm{Ca}^{2+}$ concentration to the point at which $\mathrm{Ca}^{2+}$ overload ensues. This overload increases ROS production and, once the antioxidant system is overrun, causes $\Delta \Psi \mathrm{m}$ loss (by $\mathrm{mPTP}$ and IMAC-mediated mechanisms), decreased ATP production, and MPTP opening. Furthermore, increased ROS oxidates the $\mathrm{mCU}$, along with possible phosphorylation from AMPK, secondary to low ATP synthesis, which further increases $\mathrm{Ca}^{2+}$ transport into the matrix, creating a positive feedback loop for more ROS production. ROS can also affect nearby structures, such as SERCA, which decreases its effectiveness in pumping $\mathrm{Ca}^{2+}$ back into the SR. This translates into reduced SR content and higher cytoplasmic $\mathrm{Ca}^{2+}$ concentrations, reducing contractility. $\mathrm{Ca}^{2+}$ can then activate CAMKII, which can also be activated by mitochondrial ROS. CAMKII can then autophosphorylate itself to stay in a permanent active form. RyR2 is activated by CAMKII phosphorylation and ROS, which causes its regulator protein, calstabin, to detach from the RyR2 complex. This increased sensibilization promotes stochastic opening with subsequent $\mathrm{Ca}^{2+}$ leaks and $\mathrm{Ca}^{2+}$ waves, further increasing mitochondrial $\mathrm{Ca}^{2+}$ transport. On the sarcolemma, the oxidation of LTCCs increase the amount of $\mathrm{Ca}^{2+}$ that enters the cardiomyocyte upon activation. A reduction in the ATP concentration caused by mitochondrial dysfunction opens sarcKATP channels, reducing the cardiomyocyte's bathmotropism and dromotropism. The constantly high $\mathrm{Ca}^{2+}$ concentration also activates NCX, slowly depolarizing the cell and promoting unsolicited depolarizations and APs. Finally, connexin 43 translocation or Panx1 opening due to increased $\mathrm{Ca}^{2+}$ and ROS translocates ATP and adenine into the extracellular matrix, where they activate signaling pathways for TGF- $\beta 1$ expression, with subsequent fibrosis. This represents two pathways to cardiac tissue fibrosis: cell death-dependent and TGF- $\beta 1$-dependent. Both have the same outcomes, creating patches of slow conduction and promoting reentry. All these effects make a suitable environment for conduction dysfunction and arrhythmia generation. Up until now, classic antiarrhythmic drugs' main mechanism known to prevent arrhythmias does not include the mitochondrial-SR interconnection. However, they have effects on either of these organelles (Sugiyama et al., 1985; Sano et al., 1990; Deng and Zhang, 1993; Tsutsumi et al., 2001; Afanas'ev et al., 2002; Ugdyzhekova et al., 2005; Wang et al., 2007; Bannister et al., 2015; Kryshtal et al., 2020). Flecainide can inhibit RyR2 opening, although its relevance in preventing arrhythmias is still controversial (Bannister et al., 2015; Kryshtal et al., 2020). Lidocaine might inhibit mitoK $\mathrm{K}_{\mathrm{ATP}}$ channels, as shown in isolated cardiomyocytes utilizing a mitochondrial redox state reporter as a surrogate for mitoK $K_{\text {ATP }}$ opening (Tsutsumi et al., 2001), and prevents mitochondrial $\mathrm{Ca}^{2+}$ overload in a model of closed-chest
VF and resuscitation (Wang et al., 2007), but the widely reported mechanism of action of Lidocaine is prolonging the inactivation of the fast voltage-gated $\mathrm{Na}^{+}$channels, inhibiting spontaneous depolarization (Sheu and Lederer, 1985). Amiodarone was found to preserve mitochondrial respiration after ischemia in a model of ischemia-induced ventricular arrhythmias (Sano et al., 1990) and was shown to potentiate the SR's ability to accumulate $\mathrm{Ca}^{2+}$ in either rat or isolated myocardial strips from coronary heart disease patients (Afanas'ev et al., 2002; Ugdyzhekova et al., 2005). However, its reported action is by inhibiting the $\mathrm{I}_{\mathrm{Kr}}$ current, prolonging phase 3 of the AP. Finally, Verapamil blocks voltage-dependent $\mathrm{Ca}^{2+}$ channels, decreasing impulse conduction through the AV node, but has been reported that indirectly prevents mitochondrial $\mathrm{Ca}^{2+}$ accumulation (Sugiyama et al., 1985) and reduce mitochondrial oxidative stress in the context of ischemia-reperfusion by a lower MDA content in mitochondria (Deng and Zhang, 1993). These effects could be additional antiarrhythmic mechanisms that should be further addressed to fully understand and assess their physiological relevance for future antiarrhythmic therapy developing.

\section{CONCLUSION}

In the last few years, there have been considerable advancements in understanding the molecular mechanisms of cardiac disease, especially arrhythmogenesis. These advancements laid the path for developing better therapeutic strategies that were first ignored or disregarded as potentially ineffective. Gaining a better understanding of the molecular mechanisms involved in arrhythmia generation provides insights that could lead to new therapeutic strategies. It may be time to expand our viewpoint and explore the crosstalk taking place between mitochondriaSR interconnection that seem to orchestrate $\mathrm{Ca}^{2+}$ homeostasis instead of focusing on a single cellular component. This may be the most effective way to prevent arrhythmogenesis, by tackling the central mechanism that leads to different cellular malfunctions.

\section{AUTHOR CONTRIBUTIONS}

GG-R conception and designed the review. FS-R, RR-M, and GG-R analyzed the literature and drafted the manuscript. GG-R and RR-M contributed to critial review of the manuscript. This work was submitted in partial fulfillment of the requirements for the Ph.D. degree (FS-R) for the Doctorate in Biomedical Sciences. Tecnologico de Monterrey. All authors contributed to the article and approved the submitted version.

\section{FUNDING}

This work was supported by the Cardiovascular Medicine Research Group - Tecnologico de Monterrey and the Consejo Nacional de Ciencia y Tecnología, Mexico (CONACYT) Grants 256577, 258197, 0682, and A1-S-43883. FS-R was supported by a doctoral Fellowship from CONACYT. 


\section{REFERENCES}

Adabag, A. S., Luepker, R. V., Roger, V. L., and Gersh, B. J. (2010). Sudden cardiac death: epidemiology and risk factors. Nat. Rev. Cardiol. 7, 216-225.

Afanas'ev, S. A., Lukavskaya, I. A., Kandinskii, M. L., and Medvedev, M. A. (2002). Effect of amiodarone on functional state of sarcoplasmic reticulum in rat myocardium. Bull. Exp. Biol. Med. 133, 205-207.

Ai, X., Curran, J. W., Shannon, T. R., Bers, D. M., and Pogwizd, S. M. (2005). $\mathrm{Ca} 2+/$ calmodulin-dependent protein kinase modulates cardiac ryanodine receptor phosphorylation and sarcoplasmic reticulum $\mathrm{Ca} 2+$ leak in heart failure. Circ. Res. 97, 1314-1322. doi: 10.1161/01.res.0000194329.41863.89

Akar, F. G., Aon, M. A., Tomaselli, G. F., and O'Rourke, B. (2005). The mitochondrial origin of postischemic arrhythmias. J. Clin. Invest. 115, 35273535. doi: $10.1172 /$ jci25371

Alfadda, A. A., and Sallam, R. M. (2012). Reactive oxygen species in health and disease. J. Biomed. Biotechnol. 2012:936486.

Alves-Figueiredo, H., Silva-Platas, C., Lozano, O., Vázquez-Garza, E., GuerreroBeltrán, C. E., Zarain-Herzberg, A., et al. (2021). A systematic review of post-translational modifications in the mitochondrial permeability transition pore complex associated with cardiac diseases. Biochim. Biophys. Acta Mol. Basis Dis. 1867:165992. doi: 10.1016/j.bbadis.2020.165992

Anzai, K., Ogawa, K., Kuniyasu, A., Ozawa, T., Yamamoto, H., and Nakayama, H. (1998). Effects of hydroxyl radical and sulfhydryl reagents on the open probability of the purified cardiac ryanodine receptor channel incorporated into planar lipid bilayers. Biochem. Biophys. Res. Commun. 249, 938-942. doi: 10.1006/bbrc.1998.9244

Aon, M. A., Cortassa, S., Marbán, E., and O’Rourke, B. (2003). Synchronized whole cell oscillations in mitochondrial metabolism triggered by a local release of reactive oxygen species in cardiac myocytes. J. Biol. Chem. 278, 44735-44744. doi: 10.1074/jbc.m302673200

Argaud, L., Cour, M., Dubien, P. Y., Giraud, F., Jossan, C., Riche, B., et al. (2016). Effect of cyclosporine in nonshockable out-of-hospital cardiac arrest: the CYRUS randomized clinical trial. JAMA Cardiol. 1, 557-565. doi: 10.1001/ jamacardio.2016.1701

Arteaga, D., Odor, A., López, R. M., Contreras, G., Pichardo, J., García, E., et al. (1992). Impairment by cyclosporin A of reperfusion-induced arrhythmias. Life Sci. 51, 1127-1134. doi: 10.1016/0024-3205(92)90514-p

Askari, G., Ghiasvand, R., Feizi, A., Ghanadian, S. M., and Karimian, J. (2012). The effect of quercetin supplementation on selected markers of inflammation and oxidative stress. J. Res. Med. Sci. 17, 637-641.

Avula, U. M. R., Hernandez, J. J., Yamazaki, M., Valdivia, C. R., Chu, A., RojasPena, A., et al. (2018). Atrial infarction-induced spontaneous focal discharges and atrial fibrillation in sheep: role of dantrolene-sensitive aberrant ryanodine receptor calcium release. Circ. Arrhythm. Electrophysiol. 11:e005659. doi: 10. 1161/CIRCEP.117.005659

Ayoub, I. M., Radhakrishnan, J., and Gazmuri, R. J. (2017). In vivo opening of the mitochondrial permeability transition pore in a rat model of ventricular fibrillation and closed-chest resuscitation. Am. J. Transl. Res. 9, 3345-3359.

Bannister, M. L., Thomas, N. L., Sikkel, M. B., Mukherjee, S., Maxwell, C., MacLeod, K. T., et al. (2015). The mechanism of flecainide action in CPVT does not involve a direct effect on RyR2. Circ. Res. 116, 1324-1335. doi: 10.1161/ circresaha.116.305347

Bassani, J. W., Bassani, R. A., and Bers, D. M. (1994). Relaxation in rabbit and rat cardiac cells: species-dependent differences in cellular mechanisms. J. Physiol. 476, 279-293. doi: 10.1113/jphysiol.1994.sp020130

Belevych, A. E., Terentyev, D., Terentyeva, R., Ho, H. T., Gyorke, I., Bonilla, I. M., et al. (2012). Shortened Ca2+ signaling refractoriness underlies cellular arrhythmogenesis in a postinfarction model of sudden cardiac death. Circ. Res. 110, 569-577. doi: 10.1161/circresaha.111.260455

Bellinger, A. M., Reiken, S., Dura, M., Murphy, P. W., Deng, S. X., Landry, D. W., et al. (2008). Remodeling of ryanodine receptor complex causes "leaky" channels: a molecular mechanism for decreased exercise capacity. Proc. Natl. Acad. Sci. U.S.A. 105, 2198-2202. doi: 10.1073/pnas.0711074105

Bers, D. M. (2002). Cardiac excitation-contraction coupling. Nature 415, 198-205. Beuckelmann, D. J., and Erdmann, E. (1992). Ca(2+)-currents and intracellular $[\mathrm{Ca} 2+] \mathrm{i}-$ transients in single ventricular myocytes isolated from terminally failing human myocardium. Basic Res. Cardiol. 87(Suppl. 1), 235-243. doi: 10.1007/978-3-642-72474-9_19
Beutner, G., Sharma, V. K., Giovannucci, D. R., Yule, D. I., and Sheu, S. S. (2001). Identification of a ryanodine receptor in rat heart mitochondria. J. Biol. Chem. 276, 21482-21488. doi: 10.1074/jbc.m101486200

Boengler, K., Stahlhofen, S., van de Sand, A., Gres, P., Ruiz-Meana, M., GarciaDorado, D., et al. (2009). Presence of connexin 43 in subsarcolemmal, but not in interfibrillar cardiomyocyte mitochondria. Basic Res. Cardiol. 104, 141-147. doi: 10.1007/s00395-009-0007-5

Böhm, A., Tothova, L., Urban, L., Slezak, P., Bacharova, L., Musil, P., et al. (2016). The relation between oxidative stress biomarkers and atrial fibrillation after pulmonary veins isolation. J. Electrocardiol. 49, 423-428. doi: 10.1016/j. jelectrocard.2016.03.007

Boraso, A., and Williams, A. J. (1994). Modification of the gating of the cardiac sarcoplasmic reticulum $\mathrm{Ca}(2+)$-release channel by $\mathrm{H}_{2} \mathrm{O}_{2}$ and dithiothreitol. Am. J. Physiol. 267(3 Pt 2), H1010-H1016.

Bovo, E., Lipsius, S. L., and Zima, A. V. (2012). Reactive oxygen species contribute to the development of arrhythmogenic $\mathrm{Ca}^{2+}$ waves during $\beta$-adrenergic receptor stimulation in rabbit cardiomyocytes. J. Physiol. 590, 3291-3304. doi: 10.1113/jphysiol.2012.230748

Brown, D. A., Aon, M. A., Akar, F. G., Liu, T., Sorarrain, N., and O’Rourke, B. (2008). Effects of 4'-chlorodiazepam on cellular excitation-contraction coupling and ischaemia-reperfusion injury in rabbit heart. Cardiovasc. Res. 79, 141-149. doi: 10.1093/cvr/cvn053

Bugger, H., and Abel, E. D. (2014). Molecular mechanisms of diabetic cardiomyopathy. Diabetologia 57, 660-671. doi: 10.1007/s00125-014-3171-6

Cerrone, M., van Opbergen, C. J. M., Malkani, K., Irrera, N., Zhang, M., Van Veen, T. A. B., et al. (2018). Blockade of the adenosine 2A receptor mitigates the cardiomyopathy induced by loss of Plakophilin-2 expression. Front. Physiol. 9:1750. doi: 10.3389/fphys.2018.01750

Chelu, M. G., Sarma, S., Sood, S., Wang, S., van Oort, R. J., Skapura, D. G., et al. (2009). Calmodulin kinase II-mediated sarcoplasmic reticulum $\mathrm{Ca}^{2+}$ leak promotes atrial fibrillation in mice. J. Clin. Invest. 119, 1940-1951. doi: $10.1172 /$ jci37059

Cheng, H., and Lederer, W. J. (2008). Calcium sparks. Physiol. Rev. 88, 1491-1545.

Cho, J., Won, K., Wu, D., Soong, Y., Liu, S., Szeto, H. H., et al. (2007). Potent mitochondria-targeted peptides reduce myocardial infarction in rats. Coron. Artery Dis. 18, 215-220. doi: 10.1097/01.mca.0000236285.71683.b6

Chu, G., Luo, W., Slack, J. P., Tilgmann, C., Sweet, W. E., Spindler, M., et al. (1996). Compensatory mechanisms associated with the hyperdynamic function of phospholamban-deficient mouse hearts. Circ. Res. 79, 1064-1076. doi: 10. 1161/01.res.79.6.1064

Cooper, L. L., Li, W., Lu, Y., Centracchio, J., Terentyeva, R., Koren, G., et al. (2013). Redox modification of ryanodine receptors by mitochondriaderived reactive oxygen species contributes to aberrant $\mathrm{Ca} 2+$ handling in ageing rabbit hearts. J. Physiol. 591, 5895-5911. doi: 10.1113/jphysiol.2013.260 521

Crompton, M. (1990). "The role of Ca2+ in the function and dysfunction of heart mitochondria," in Calcium and the Heart, ed. G. A. Langer, (New York, NY: Raven Press), 167-198.

Crompton, M., Ellinger, H., and Costi, A. (1988). Inhibition by cyclosporin A of a $\mathrm{Ca} 2+$-dependent pore in heart mitochondria activated by inorganic phosphate and oxidative stress. Biochem. J. 255, 357-360.

Csordás, G., Renken, C., Várnai, P., Walter, L., Weaver, D., Buttle, K. F., et al. (2006). Structural and functional features and significance of the physical linkage between ER and mitochondria. J. Cell Biol. 174, 915-921. doi: 10.1083/jcb. 200604016

Davidson, A. M., and Halestrap, A. P. (1990). Partial inhibition by cyclosporin A of the swelling of liver mitochondria in vivo and in vitro induced by submicromolar $[\mathrm{Ca} 2+]$, but not by butyrate. Evidence for two distinct swelling mechanisms. Biochem. J. 268, 147-152. doi: 10.1042/bj2680147

de Brito, O. M., and Scorrano, L. (2008). Mitofusin 2 tethers endoplasmic reticulum to mitochondria. Nature 456, 605-610. doi: 10.1038/nature07534

De Vos, K. J., Mórotz, G. M., Stoica, R., Tudor, E. L., Lau, K. F., Ackerley, S., et al. (2012). VAPB interacts with the mitochondrial protein PTPIP51 to regulate calcium homeostasis. Hum. Mol. Genet. 21, 1299-1311. doi: 10.1093/hmg/ ddr559

Deng, Y., and Zhang, Z. S. (1993). Protective effects of combination of chlorpromazine and verapamil on ischemia-reperfusion induced injury in rat myocardium. Yao Xue Xue Bao 28, 561-566. 
Dey, S., DeMazumder, D., Sidor, A., Foster, D. B., and O'Rourke, B. (2018). Mitochondrial ROS drive sudden cardiac death and chronic proteome remodeling in heart failure. Cir.c Res. 123, 356-371. doi: 10.1161/circresaha. 118.312708

Di Pasquale, E., Lodola, F., Miragoli, M., Denegri, M., Avelino-Cruz, J. E., Buonocore, M., et al. (2013). CaMKII inhibition rectifies arrhythmic phenotype in a patient-specific model of catecholaminergic polymorphic ventricular tachycardia. Cell Death Dis. 4:e843. doi: 10.1038/cddis.2013.369

Dolmatova, E., Spagnol, G., Boassa, D., Baum, J. R., Keith, K., Ambrosi, C., et al. (2012). Cardiomyocyte ATP release through pannexin 1 aids in early fibroblast activation. Am. J. Physiol. Heart Circ. Physiol. 303, H1208-H1218.

Dong, Z., Shanmughapriya, S., Tomar, D., Siddiqui, N., Lynch, S., Nemani, N., et al. (2017). Mitochondrial $\mathrm{Ca}(2+)$ uniporter is a mitochondrial luminal redox sensor that augments MCU channel activity. Mol. Cell 65, 1014-1028.e7.

Dries, E., Santiago, D. J., Gilbert, G., Lenaerts, I., Vandenberk, B., Nagaraju, C. K., et al. (2018). Hyperactive ryanodine receptors in human heart failure and ischaemic cardiomyopathy reside outside of couplons. Cardiovasc. Res. 114, 1512-1524. doi: 10.1093/cvr/cvy088

Eijsbouts, S. C., Majidi, M., van Zandvoort, M., and Allessie, M. A. (2003). Effects of acute atrial dilation on heterogeneity in conduction in the isolated rabbit heart. J. Cardiovasc. Electrophysiol. 14, 269-278. doi: 10.1046/j.1540-8167.2003. 02280.x

Eisner, D. A., Caldwell, J. L., Kistamas, K., and Trafford, A. W. (2017). Calcium and excitation-contraction coupling in the heart. Circ. Res. 121, 181-195. doi: 10.1016/b978-012436570-4/50007-8

Elgebaly, S. A., Allam, M. E., Houser, S., Hashmi, F., Forouhar, F., and Miano, D. (1993). Cyclocreatine inhibits neutrophil accumulation in the myocardium of a canine model of coronary artery occlusion and reperfusion. J. Pharmacol. Exp. Ther. 266, 1670-1677.

Elgebaly, S. A., Allam, M. E., Rossomando, E. F., Cordis, G. A., Forouhar, F., Farghaly, A., et al. (1990). Cyclocreatine inhibits the production of neutrophil chemotactic factors from isolated hearts. Am. J. Pathol. 137, 1233-1241.

Elgebaly, S. A., Wei, Z., Tyles, E., Elkerm, A. F., Houser, S. L., Gillies, C., et al. (1994). Enhancement of the recovery of rat hearts after prolonged cold storage by cyclocreatine phosphate. Transplantation 57, 803-806. doi: 10.1097/ 00007890-199403270-00005

El-Hamamsy, I., Stevens, L. M., Carrier, M., Pellerin, M., Bouchard, D., Demers, P., et al. (2007). Effect of intravenous $\mathrm{N}$-acetylcysteine on outcomes after coronary artery bypass surgery: a randomized, double-blind, placebo-controlled clinical trial. J. Thorac. Cardiovasc. Surg. 133, 7-12. doi: 10.1016/j.jtcvs.2006.05.070

Erickson, J. R., Joiner, M. L., Guan, X., Kutschke, W., Yang, J., Oddis, C. V., et al. (2008). A dynamic pathway for calcium-independent activation of CaMKII by methionine oxidation. Cell 133, 462-474. doi: 10.1016/j.cell.2008.02.048

Erickson, J. R., Pereira, L., Wang, L., Han, G., Ferguson, A., Dao, K., et al. (2013). Diabetic hyperglycaemia activates CaMKII and arrhythmias by O-linked glycosylation. Nature 502, 372-376. doi: 10.1038/nature12537

Fauconnier, J., Thireau, J., Reiken, S., Cassan, C., Richard, S., Matecki, S., et al. (2010). Leaky RyR2 trigger ventricular arrhythmias in Duchenne muscular dystrophy. Proc. Natl. Acad. Sci. U.S.A. 107, 1559-1564. doi: 10.1073/pnas. 0908540107

Federico, M., Valverde, C. A., Mattiazzi, A., and Palomeque, J. (2019). Unbalance between sarcoplasmic reticulum $\mathrm{Ca}^{2+}$ uptake and release: a first step toward $\mathrm{Ca}^{2+}$ triggered arrhythmias and cardiac damage. Front. Physiol. 10:1630. doi: 10.3389/fphys.2019.01630

Fernandez-Sada, E., Silva-Platas, C., Villegas, C. A., Rivero, S. L., Willis, B. C., Garcia, N., et al. (2014). Cardiac responses to beta-adrenoceptor stimulation is partly dependent on mitochondrial calcium uniporter activity. Br. J. Pharmacol. 171, 4207-4221. doi: 10.1111/bph.12684

Fernandez-Sanz, C., Ruiz-Meana, M., Miro-Casas, E., Nuñez, E., Castellano, J., Loureiro, M., et al. (2014). Defective sarcoplasmic reticulum-mitochondria calcium exchange in aged mouse myocardium. Cell Death Dis. 5:e1573. doi: 10.1038/cddis.2014.526

Fill, M., and Copello, J. A. (2002). Ryanodine receptor calcium release channels. Physiol. Rev. 82, 893-922. doi: 10.1016/s0006-3495(95)80265-1

Fischer, E., Gottschalk, A., and Schüler, C. (2017). An optogenetic arrhythmia model to study catecholaminergic polymorphic ventricular tachycardia mutations. Sci. Rep. 7:17514. doi: 10.1038/s41598-017-17819-8
Flores-Mateo, G., Navas-Acien, A., Pastor-Barriuso, R., and Guallar, E. (2006). Selenium and coronary heart disease: a meta-analysis. Am. J. Clin. Nutr. 84, 762-773. doi: 10.1093/ajcn/84.4.762

Formica, J. V., and Regelson, W. (1995). Review of the biology of Quercetin and related bioflavonoids. Food Chem. Toxicol. 33, 1061-1080. doi: 10.1016/02786915(95)00077-1

Friedman, J. R., Lackner, L. L., West, M., DiBenedetto, J. R., Nunnari, J., and Voeltz, G. K. (2011). ER tubules mark sites of mitochondrial division. Science 334, 358-362. doi: $10.1126 /$ science. 1207385

Frommeyer, G., Krawczyk, J., Ellermann, C., Bogeholz, N., Kochhauser, S., Dechering, D. G., et al. (2018). Ryanodine-receptor inhibition by dantrolene effectively suppresses ventricular arrhythmias in an ex vivo model of long-QT syndrome. J. Cardiovasc. Electrophysiol. 29, 471-476. doi: 10.1111/jce.13412

Gambardella, J., Sorriento, D., Ciccarelli, M., Del Giudice, C., Fiordelisi, A., Napolitano, L., et al. (2017). Functional role of mitochondria in arrhythmogenesis. Adv. Exp. Med. Biol. 982, 191-202. doi: 10.1007/978-3-31955330-6_10

Garciarena, C. D., Caldiz, C. I., Correa, M. V., Schinella, G. R., Mosca, S. M., Chiappe de Cingolani, G. E., et al. (2008). $\mathrm{Na}^{+} / \mathrm{H}^{+}$exchanger-1 inhibitors decrease myocardial superoxide production via direct mitochondrial action. J. Appl. Physiol. 105, 1706-1713. doi: 10.1152/japplphysiol.90616.2008

García-Rivas, G. D. J., Guerrero-Hernández, A., Guerrero-Serna, G., RodríguezZavala, J. S., and Zazueta, C. (2005). Inhibition of the mitochondrial calcium uniporter by the oxo-bridged dinuclear ruthenium amine complex (Ru360) prevents from irreversible injury in postischemic rat heart. FEBS J. 272, 34773488. doi: $10.1111 / j .1742-4658.2005 .04771 . x$

Garcia-Rivas Gde, J., Carvajal, K., Correa, F., and Zazueta, C. (2006). $\mathrm{Ru}_{360}$, a specific mitochondrial calcium uptake inhibitor, improves cardiac post-ischaemic functional recovery in rats in vivo. Br. J. Pharmacol. 149, 829-837. doi: 10.1038/s.bjp.0706932

Giorgi, C., Marchi, S., and Pinton, P. (2018). The machineries, regulation and cellular functions of mitochondrial calcium. Nat. Rev. Mol. Cell Biol. 19, 713-730. doi: 10.1038/s41580-018-0052-8

Glancy, B., and Balaban, R. S. (2012). Role of mitochondrial Ca2+ in the regulation of cellular energetics. Biochemistry 51, 2959-2973. doi: 10.1021/bi2018909

Gonca, E., Rapposelli, S., Darıcı, F., Digiacomo, M., and Y $\iota$ lmaz, Z. (2016). Antiarrhythmic activity of a new spiro-cyclic benzopyran activator of the cardiac mitochondrial ATP dependent potassium channels. Arch. Pharm. Res. 39, 1212-1222. doi: 10.1007/s12272-016-0779-8

Gordan, R., Fefelova, N., Gwathmey, J. K., and Xie, L. H. (2016). Involvement of mitochondrial permeability transition pore (mPTP) in cardiac arrhythmias: Evidence from cyclophilin D knockout mice. Cell Calcium 60, 363-372. doi: 10.1016/j.ceca.2016.09.001

Gordan, R., Fefelova, N., Gwathmey, J. K., and Xie, L. H. (2020). Iron overload, oxidative stress and calcium mishandling in Cardiomyocytes: role of the mitochondrial permeability transition pore. Antioxidants 9:758. doi: 10.3390/ antiox 9080758

Greenberg, B., Butler, J., Felker, G. M., Ponikowski, P., Voors, A. A., Desai, A. S., et al. (2016). Calcium upregulation by percutaneous administration of gene therapy in patients with cardiac disease (CUPID 2): a randomised, multinational, double-blind, placebo-controlled, phase $2 \mathrm{~b}$ trial. Lancet 387 , 1178-1186. doi: 10.1016/s0140-6736(16)00082-9

Griffiths, E. J., and Halestrap, A. P. (1991). Further evidence that cyclosporin A protects mitochondria from calcium overload by inhibiting a matrix peptidylprolyl cis-trans isomerase. Implications for the immunosuppressive and toxic effects of cyclosporin. Biochem. J. 274(Pt 2), 611-614. doi: 10.1042/bj2740611

Hamilton, S., Terentyeva, R., Kim, T. Y., Bronk, P., Clements, R. T., O-Uchi, J., et al. (2018). Pharmacological modulation of mitochondrial $\mathrm{Ca}^{2+}$ content regulates sarcoplasmic reticulum $\mathrm{Ca}^{2+}$ release via oxidation of the ryanodine receptor by mitochondria-derived reactive oxygen species. Front. Physiol. 9:1831. doi: 10.3389/fphys.2018.01831

Hamilton, S., Terentyeva, R., Martin, B., Perger, F., Li, J., Stepanov, A., et al. (2020). Increased RyR2 activity is exacerbated by calcium leak-induced mitochondrial ROS. Basic Res. Cardiol. 115:38. doi: 10.1007/s00395-020-0797-z

Harada, M., Nattel, S. N., and Nattel, S. (2012). AMP-activated protein kinase: potential role in cardiac electrophysiology and arrhythmias. Circ. Arrhythm. Electrophysiol. 5, 860-867. doi: 10.1161/circep.112.972265 
Harman, D. (1956). Aging: a theory based on free radical and radiation chemistry. J. Gerontol. 11, 298-300. doi: 10.1093/geronj/11.3.298

Harris, D. A., and Das, A. M. (1991). Control of mitochondrial ATP synthesis in the heart. Biochem. J. 280(Pt 3), 561-573. doi: 10.1042/bj2800561

Hartmann, N., Pabel, S., Herting, J., Schatter, F., Renner, A., Gummert, J., et al. (2017). Antiarrhythmic effects of dantrolene in human diseased cardiomyocytes. Heart Rhythm. 14, 412-419. doi: 10.1016/j.hrthm.2016.09.014

Hernández-Esquivel, L., Pavón, N., Buelna-Chontal, M., González-Pacheco, H., Belmont, J., and Chávez, E. (2014). Citicoline (CDP-choline) protects myocardium from ischemia/reperfusion injury via inhibiting mitochondrial permeability transition. Life Sci. 96, 53-58. doi: 10.1016/j.lfs.2013.12.026

Herraiz-Martínez, A., Álvarez-García, J., Llach, A., Molina, C. E., Fernandes, J., Ferrero-Gregori, A., et al. (2015). Ageing is associated with deterioration of calcium homeostasis in isolated human right atrial myocytes. Cardiovasc. Res. 106, 76-86. doi: 10.1093/cvr/cvv046

Hicks, J. J., Montes-Cortes, D. H., Cruz-Dominguez, M. P., Medina-Santillan, R., and Olivares-Corichi, I. M. (2007). Antioxidants decrease reperfusion induced arrhythmias in myocardial infarction with ST-elevation. Front. Biosci. 12, 20292037. doi: $10.2741 / 2208$

Hobai, I. A., Buys, E. S., Morse, J. C., Edgecomb, J., Weiss, E. H., Armoundas, A. A., et al. (2013). SERCA Cys674 sulphonylation and inhibition of L-type Ca2+ influx contribute to cardiac dysfunction in endotoxemic mice, independent of cGMP synthesis. Am. J. Physiol. Heart Circ. Physiol. 305, H1189-H1200.

Horjus, D. L., Oudman, I., van Montfrans, G. A., and Brewster, L. M. (2011). Creatine and creatine analogues in hypertension and cardiovascular disease. Cochrane Database Syst. Rev. 2011:Cd005184.

Houser, S. L., Elkerm, A. F., Wei, Z., Doyle, K., Houser, D., Liu, X. K., et al. (1995). Enhancement of cardiac function by cyclocreatine in models of cardiopulmonary bypass. J. Mol. Cell Cardiol. 27, 1065-1073. doi: 10.1016/ 0022-2828(95)90075-6

Hung, L. M., Chen, J. K., Huang, S. S., Lee, R. S., and Su, M. J. (2000). Cardioprotective effect of resveratrol, a natural antioxidant derived from grapes. Cardiovasc. Res. 47, 549-555. doi: 10.1016/s0008-6363(00)00102-4

Ito, K., Shigematsu, S., Sato, T., Abe, T., Li, Y., and Arita, M. (2000). JTV519, a novel cardioprotective agent, improves the contractile recovery after ischaemia-reperfusion in coronary perfused guinea-pig ventricular muscles. $\mathrm{Br}$. J. Pharmacol. 130, 767-776. doi: 10.1038/sj.bjp.0703373

Jansen, J. A., van Veen, T. A., de Bakker, J. M., and van Rijen, H. V. (2010), Cardiac connexins and impulse propagation. J. Mol. Cell Cardiol. 48, 76-82. doi: 10.1016/j.yjmcc.2009.08.018

Jaski, B. E., Jessup, M. L., Mancini, D. M., Cappola, T. P., Pauly, D. F., Greenberg, B., et al. (2009). Calcium upregulation by percutaneous administration of gene therapy in cardiac disease (CUPID Trial), a first-in-human phase $1 / 2$ clinical trial. J. Card Fail. 15, 171-181. doi: 10.1016/j.cardfail.2009.01.013

Jeong, E. M., Liu, M., Sturdy, M., Gao, G., Varghese, S. T., Sovari, A. A., et al. (2012). Metabolic stress, reactive oxygen species, and arrhythmia. J. Mol. Cell Cardiol. $52,454-463$.

Jessup, M., Greenberg, B., Mancini, D., Cappola, T., Pauly, D. F., Jaski, B., et al. (2011). Calcium upregulation by percutaneous administration of Gene Therapy in Cardiac Disease (CUPID): a phase 2 trial of intracoronary gene therapy of sarcoplasmic reticulum $\mathrm{Ca} 2+-$ ATPase in patients with advanced heart failure. Circulation 124, 304-313. doi: 10.1161/circulationaha.111.022889

Jin, J. K., Blackwood, E. A., Azizi, K., Thuerauf, D. J., Fahem, A. G., Hofmann, C., et al. (2017). ATF6 decreases myocardial ischemia/reperfusion damage and links ER Stress and oxidative stress signaling pathways in the heart. Circ. Res. 120, 862-875. doi: 10.1161/circresaha.116.310266

Joiner, M. L., and Koval, O. M. (2014). CaMKII and stress mix it up in mitochondria. Front. Pharmacol. 5:67. doi: 10.3389/fphar.2014.00067

Joiner, M. L., Koval, O. M., Li, J., He, B. J., Allamargot, C., Gao, Z., et al. (2012). CaMKII determines mitochondrial stress responses in heart. Nature 491, 269-273. doi: 10.1038/nature11444

Kazak, L., Chouchani, E. T., Stavrovskaya, I. G., Lu, G. Z., Jedrychowski, M. P., Egan, D. F., et al. (2017). UCP1 deficiency causes brown fat respiratory chain depletion and sensitizes mitochondria to calcium overload-induced dysfunction. Proc. Natl. Acad. Sci. U.S.A. 114, 7981-7986. doi: 10.1073/pnas. 1705406114

Khoo, M. S., Li, J., Singh, M. V., Yang, Y., Kannankeril, P., Wu, Y., et al. (2006). Death, cardiac dysfunction, and arrhythmias are increased by calmodulin kinase II in calcineurin cardiomyopathy. Circulation 114, 1352-1359. doi: 10.1161/circulationaha.106.644583

Khurshid, S., Choi, S. H., Weng, L. C., Wang, E. Y., Trinquart, L., Benjamin, E. J., et al. (2018). Frequency of cardiac rhythm abnormalities in a half million adults. Circ. Arrhythm. Electrophysiol. 11:e006273. doi: 10.1161/circep.118.006273

Kim, J. C., Pérez-Hernández, M., Alvarado, F. J., Maurya, S. R., Montnach, J., Yin, Y., et al. (2019). Disruption of $\mathrm{Ca}(2+)(\mathrm{i})$ homeostasis and connexin 43 hemichannel function in the right ventricle precedes overt arrhythmogenic cardiomyopathy in Plakophilin-2-deficient mice. Circulation 140, 1015-1030. doi: 10.1161/circulationaha.119.039710

Kim, Y. H., Lim, D. S., Lee, J. H., Shim, W. J., Ro, Y. M., Park, G. H., et al. (2003). Gene expression profiling of oxidative stress on atrial fibrillation in humans. Exp. Mol. Med. 35, 336-349. doi: 10.1038/emm.2003.45

Kimura, H., Kawahara, K., Yamauchi, Y., and Miyaki, J. (2005). On the mechanisms for the conversion of ventricular fibrillation to tachycardia by perfusion with ruthenium red. J. Electrocardiol. 38, 364-370. doi: 10.1016/j.jelectrocard.2005. 05.007

Kirchhefer, U., Schmitz, W., Scholz, H., and Neumann, J. (1999). Activity of cAMPdependent protein kinase and $\mathrm{Ca} 2+/$ calmodulin-dependent protein kinase in failing and nonfailing human hearts. Cardiovasc. Res. 42, 254-261. doi: 10. 1016/s0008-6363(98)00296-x

Kirchhoff, S., Nelles, E., Hagendorff, A., Krüger, O., Traub, O., and Willecke, K. (1998). Reduced cardiac conduction velocity and predisposition to arrhythmias in connexin40-deficient mice. Curr. Biol. 8, 299-302. doi: 10.1016/s09609822(98)70114-9

Knottnerus, S. J. G., Mengarelli, I., Wüst, R. C. I., Baartscheer, A., Bleeker, J. C., Coronel, R., et al. (2020). Electrophysiological abnormalities in VLCAD Deficient hiPSC-Cardiomyocytes can be improved by lowering accumulation of fatty acid oxidation intermediates. Int. J. Mol. Sci. 21:2589. doi: 10.3390/ ijms21072589

Kobayashi, S., Bannister, M. L., Gangopadhyay, J. P., Hamada, T., Parness, J., and Ikemoto, N. (2005). Dantrolene stabilizes domain interactions within the ryanodine receptor. J. Biol. Chem. 280, 6580-6587. doi: 10.1074/jbc. m408375200

Kobayashi, S., Yano, M., Uchinoumi, H., Suetomi, T., Susa, T., Ono, M., et al. (2010). Dantrolene, a therapeutic agent for malignant hyperthermia, inhibits catecholaminergic polymorphic ventricular tachycardia in a $\mathrm{RyR} 2^{\mathrm{R} 2474 \mathrm{~S} /+}$ knock-in mouse model. Circ. J. 74, 2579-2584. doi: 10.1253/circj.cj-100680

Kohlhaas, M., and Maack, C. (2013). Calcium release microdomains and mitochondria. Cardiovasc. Res. 98, 259-268. doi: 10.1093/cvr/cvt032

Kohno, M., Yano, M., Kobayashi, S., Doi, M., Oda, T., Tokuhisa, T., et al. (2003). A new cardioprotective agent, JTV519, improves defective channel gating of ryanodine receptor in heart failure. Am. J. Physiol. Heart Circ. Physiol. 284, H1035-H1042. doi: 10.1152/ajpheart.00722.2002

Kónya, L., Kékesi, V., Juhász-Nagy, S., and Fehér, J. (1992). The effect of superoxide dismutase in the myocardium during reperfusion in the dog. Free Radic. Biol. Med. 13, 527-532. doi: 10.1016/0891-5849(92)90147-9

Korobova, F., Ramabhadran, V., and Higgs, H. N. (2013). An actin-dependent step in mitochondrial fission mediated by the ER-associated formin INF2. Science 339, 464-467. doi: 10.1126/science. 1228360

Krul, S. P., Berger, W. R., Smit, N. W., van Amersfoorth, S. C., Driessen, A. H., van Boven, W. J., et al. (2015). Atrial fibrosis and conduction slowing in the left atrial appendage of patients undergoing thoracoscopic surgical pulmonary vein isolation for atrial fibrillation. Circ. Arrhythm. Electrophysiol. 8, 288-295. doi: 10.1161/circep.114.001752

Kryshtal, D. O., Blackwell, D., Egly, C., Smith, A. N., Batiste, S. M., Johnston, J. N., et al. (2020). RYR2 channel inhibition is the principal mechanism of Flecainide Action in CPVT. Circ Res. doi: 10.1161/CIRCRESAHA.120.316819 [Epub ahead of print].

Kushnir, A., Shan, J., Betzenhauser, M. J., Reiken, S., and Marks, A. R. (2010). Role of CaMKIIdelta phosphorylation of the cardiac ryanodine receptor in the force frequency relationship and heart failure. Proc. Natl. Acad. Sci. U.S.A. 107, 10274-10279. doi: 10.1073/pnas.1005843107

Kwong, J. Q., Lu, X., Correll, R. N., Schwanekamp, J. A., Vagnozzi, R. J., Sargent, M. A., et al. (2015). The mitochondrial calcium uniporter selectively matches metabolic output to acute contractile stress in the heart. Cell Rep. 12, 15-22. doi: 10.1016/j.celrep.2015.06.002 
Langer, G. A., and Peskoff, A. (1996). Calcium concentration and movement in the diadic cleft space of the cardiac ventricular cell. Biophys. J. 70, 1169-1182. doi: 10.1016/s0006-3495(96)79677-7

Lara-Vaca, S., Cordero-Cabra, A., Martínez-Flores, E., and Iturralde-Torres, P. (2014). Registro mexicano de fibrilación auricular (ReMeFa). Gaceta Méd. México 150, 48-59.

Larbig, R., Reda, S., Paar, V., Trost, A., Leitner, J., Weichselbaumer, S., et al. (2017). Through modulation of cardiac $\mathrm{Ca}(2+)$ handling, UCP2 affects cardiac electrophysiology and influences the susceptibility for $\mathrm{Ca}(2+)$-mediated arrhythmias. Exp. Physiol. 102, 650-662. doi: 10.1113/ep086209

Leducq, N., Bono, F., Sulpice, T., Vin, V., Janiak, P., Fur, G. L., et al. (2003). Role of peripheral benzodiazepine receptors in mitochondrial, cellular, and cardiac damage induced by oxidative stress and ischemia-reperfusion. J. Pharmacol. Exp. Ther. 306, 828-837. doi: 10.1124/jpet.103.052068

Lee, D. S., Gona, P., Albano, I., Larson, M. G., Benjamin, E. J., Levy, D., et al. (2011). A systematic assessment of causes of death after heart failure onset in the community: impact of age at death, time period, and left ventricular systolic dysfunction. Circ. Heart Fail. 4, 36-43. doi: 10.1161/circheartfailure.110.957480

Lehnart, S. E., Mongillo, M., Bellinger, A., Lindegger, N., Chen, B. X., Hsueh, W., et al. (2008). Leaky $\mathrm{Ca}^{2+}$ release channel/ryanodine receptor 2 causes seizures and sudden cardiac death in mice. J. Clin. Invest. 118, 2230-2245. doi: 10.1172/JCI35346

Lehnart, S. E., Wehrens, X. H., Laitinen, P. J., Reiken, S. R., Deng, S. X., Cheng, Z., et al. (2004a). Sudden death in familial polymorphic ventricular tachycardia associated with calcium release channel (ryanodine receptor) leak. Circulation 109, 3208-3214. doi: 10.1161/01.cir.0000132472.98675.ec

Lehnart, S. E., Wehrens, X. H., and Marks, A. R. (2004b). Calstabin deficiency, ryanodine receptors, and sudden cardiac death. Biochem. Biophys. Res. Commun. 322, 1267-1279. doi: 10.1016/j.bbrc.2004.08.032

Lemme, M., Braren, I., Prondzynski, M., Aksehirlioglu, B., Ulmer, B. M., Schulze, M. L., et al. (2019). Chronic intermittent tachypacing by an optogenetic approach induces arrhythmia vulnerability in human engineered heart tissue. Cardiovasc. Res. 116, 1487-1499. doi: 10.1093/cvr/cvz245

Lin, P. H., Lee, S. H., Su, C. P., and Wei, Y. H. (2003). Oxidative damage to mitochondrial DNA in atrial muscle of patients with atrial fibrillation. Free Radic. Biol. Med. 35, 1310-1318. doi: 10.1016/j.freeradbiomed.2003.07.002

Liu, N., Ruan, Y., Denegri, M., Bachetti, T., Li, Y., Colombi, B., et al. (2011). Calmodulin kinase II inhibition prevents arrhythmias in RyR2 ${ }^{\mathrm{R} 4496 \mathrm{C}+/-}$ mice with catecholaminergic polymorphic ventricular tachycardia. J. Mol. Cell Cardiol. 50, 214-222. doi: 10.1016/j.yjmcc.2010.10.001

Locovei, S., Wang, J., and Dahl, G. (2006). Activation of pannexin 1 channels by ATP through P2Y receptors and by cytoplasmic calcium. FEBS Lett. 580, 239-244. doi: 10.1016/j.febslet.2005.12.004

Lopez-Crisosto, C., Pennanen, C., Vasquez-Trincado, C., Morales, P. E., BravoSagua, R., Quest, A. F. G., et al. (2017). Sarcoplasmic reticulum-mitochondria communication in cardiovascular pathophysiology. Nat. Rev. Cardiol. 14, 342360. doi: $10.1038 /$ nrcardio. 2017.23

Lozano, O., Lázaro-Alfaro, A., Silva-Platas, C., Oropeza-Almazán, Y., TorresQuintanilla, A., Bernal-Ramírez, J., et al. (2019). Nanoencapsulated quercetin improves cardioprotection during hypoxia-reoxygenation injury through preservation of mitochondrial function. Oxid. Med. Cell Longev. 2019:7683051. doi: $10.1155 / 2019 / 7683051$

Lozano, O., Torres-Quintanilla, A., and García-Rivas, G. (2018). Nanomedicine for the cardiac myocyte: Where are we? J. Control Release 271, 149-165. doi: 10.1016/j.jconrel.2017.12.018

Lu, X., Ginsburg, K. S., Kettlewell, S., Bossuyt, J., Smith, G. L., and Bers, D. M. (2013). Measuring local gradients of intramitochondrial $[\mathrm{Ca}(2+)]$ in cardiac myocytes during sarcoplasmic reticulum $\mathrm{Ca}(2+)$ release. Circ. Res. 112, 424431. doi: 10.1161/circresaha.111.300501

Maack, C., and O'Rourke, B. (2007). Excitation-contraction coupling and mitochondrial energetics. Basic Res. Cardiol. 102, 369-392. doi: 10.1007/ s00395-007-0666-z

MacDonnell, S. M., García-Rivas, G., Scherman, J. A., Kubo, H., Chen, X., Valdivia, H., et al. (2008). Adrenergic regulation of cardiac contractility does not involve phosphorylation of the cardiac ryanodine receptor at serine 2808. Circ. Res. 102, e65-e72.

Maneechote, C., Palee, S., Kerdphoo, S., Jaiwongkam, T., Chattipakorn, S. C., and Chattipakorn, N. (2018). Differential temporal inhibition of mitochondrial fission by Mdivi-1 exerts effective cardioprotection in cardiac ischemia/reperfusion injury. Clin. Sci. 132, 1669-1683. doi: 10.1042/ cs20180510

Maneechote, C., Palee, S., Kerdphoo, S., Jaiwongkam, T., Chattipakorn, S. C., and Chattipakorn, N. (2019). Balancing mitochondrial dynamics via increasing mitochondrial fusion attenuates infarct size and left ventricular dysfunction in rats with cardiac ischemia/reperfusion injury. Clin. Sci. 133, 497-513. doi: $10.1042 / \mathrm{cs} 20190014$

Manor, U., Bartholomew, S., Golani, G., Christenson, E., Kozlov, M., Higgs, H., et al. (2015). A mitochondria-anchored isoform of the actin-nucleating spire protein regulates mitochondrial division. eLife 4:e08828.

Martínez-González, M., Toledo, E., Arós, F., Fiol, M., Corella, D., Salas-Salvadó, J., et al. (2014). Extravirgin olive oil consumption reduces risk of atrial fibrillation: the PREDIMED (Prevención con Dieta Mediterránea) trial. Circulation 130, 18-26. doi: 10.1161/circulationaha.113.006921

Martinvalet, D. (2018). The role of the mitochondria and the endoplasmic reticulum contact sites in the development of the immune responses. Cell Death Dis. 9:336.

Matlib, M. A., Zhou, Z., Knight, S., Ahmed, S., Choi, K. M., Krause-Bauer, J., et al. (1998). Oxygen-bridged dinuclear ruthenium amine complex specifically inhibits $\mathrm{Ca} 2+$ uptake into mitochondria in vitro and in situ in single cardiac myocytes. J. Biol. Chem. 273, 10223-10231. doi: 10.1074/jbc.273.17.10223

McCormack, J. G., and Denton, R. M. (1990). The role of mitochondrial Ca2+ transport and matrix $\mathrm{Ca} 2+$ in signal transduction in mammalian tissues. Biochim. Biophys. Acta 1018, 287-291. doi: 10.1016/0005-2728(90)90269-a

McDonald, C., Fraser, J., Shekar, K., Clarke, A., Coombes, J., Barnett, A., et al. (2016). Low preoperative selenium is associated with post-operative atrial fibrillation in patients having intermediate-risk coronary artery surgery. Eur. J. Clin. Nutr. 70, 1138-1143. doi: 10.1038/ejen.2016.125

Meissner, G. (2004). Molecular regulation of cardiac ryanodine receptor ion channel. Cell Calcium 35, 621-628. doi: 10.1016/j.ceca.2004.01.015

Menezes-Rodrigues, F. S., Tavares, J. G. P., Vasques, E. R., Errante, P. R., Araújo, E. A., Pires-Oliveira, M., et al. (2020). Cardioprotective effects of pharmacological blockade of the mitochondrial calcium uniporter on myocardial ischemia-reperfusion injury. Acta Cir. Bras. 35:e202000306.

Motloch, L. J., Hu, J., and Akar, F. G. (2015). The mitochondrial translocator protein and arrhythmogenesis in ischemic heart disease. Oxid. Med. Cell Longev. 2015:234104.

Murphy, K. R., Baggett, B., Cooper, L. L., Lu, Y., Ou, J., Sedivy, J. M., et al. (2019). Enhancing autophagy diminishes aberrant $\mathrm{Ca}^{(2+)}$ homeostasis and arrhythmogenesis in aging rabbit hearts. Front. Physiol. 10:1277. doi: 10.3389/ fphys.2019.01277

Negi, S., Shukrullah, I., Veledar, E., Bloom, H. L., Jones, D. P., and Dudley, S. C. (2011). Statin therapy for the prevention of atrial fibrillation trial (SToP AF trial). J. Cardiovasc. Electrophysiol. 22, 414-419. doi: 10.1111/j.1540-8167.2010. 01925.x

Ni, R., Cao, T., Xiong, S., Ma, J., Fan, G. C., Lacefield, J. C., et al. (2016). Therapeutic inhibition of mitochondrial reactive oxygen species with mito-TEMPO reduces diabetic cardiomyopathy. Free Radic. Biol. Med. 90, 12-23. doi: 10.1016/j. freeradbiomed.2015.11.013

Nickel, A. G., Kohlhaas, M., Bertero, E., Wilhelm, D., Wagner, M., Sequeira, V., et al. (2020). CaMKII does not control mitochondrial $\mathrm{Ca}(2+)$ uptake in cardiac myocytes. J. Physiol. 598, 1361-1376. doi: 10.1113/jp276766

Nikolaienko, R., Bovo, E., and Zima, A. V. (2018). Redox dependent modifications of ryanodine receptor: basic mechanisms and implications in heart diseases. Front. Physiol. 9:1775. doi: 10.3389/fphys.2018.01775

O’Neill, S. C., Miller, L., Hinch, R., and Eisner, D. A. (2004). Interplay between SERCA and sarcolemmal Ca2+ efflux pathways controls spontaneous release of $\mathrm{Ca} 2+$ from the sarcoplasmic reticulum in rat ventricular myocytes. J. Physiol. 559(Pt 1), 121-128. doi: 10.1113/jphysiol.2003.058917

Oropeza-Almazan, Y., and Blatter, L. A. (2020). Mitochondrial calcium uniporter complex activation protects against calcium Alternans in Atrial Myocytes. Am. J. Physiol. Heart Circ. Physiol. 319, H873-H881.

Oropeza-Almazan, Y., Vazquez-Garza, E., Chapoy-Villanueva, H., TorreAmione, G., and Garcia-Rivas, G. (2017). Small interfering RNA targeting mitochondrial calcium uniporter improves cardiomyocyte cell viability in hypoxia/reoxygenation injury by reducing calcium overload. Oxid. Med. Cell Longev. 2017:5750897. 
O'Rourke, B., and Blatter, L. A. (2009). Mitochondrial Ca2+ uptake: tortoise or hare? J. Mol. Cell Cardiol. 46, 767-774. doi: 10.1016/j.yjmcc.2008.12.011

Osbakken, M., Ito, K., Zhang, D., Ponomarenko, I., Ivanics, T., Jahngen, E. G., et al. (1992). Creatine and cyclocreatine effects on ischemic myocardium: 31P nuclear magnetic resonance evaluation of intact heart. Cardiology 80, 184-195. doi: 10.1159/000175002

Ozcan, C., Palmeri, M., Horvath, T. L., Russell, K. S., and Russell, R. R. III (2013). Role of uncoupling protein 3 in ischemia-reperfusion injury, arrhythmias, and preconditioning. Am. J. Physiol. Heart Circ. Physiol. 304, H1192-H1200.

Pain, T., Yang, X. M., Critz, S. D., Yue, Y., Nakano, A., Liu, G. S., et al. (2000). Opening of mitochondrial K(ATP) channels triggers the preconditioned state by generating free radicals. Circ. Res. 87, 460-466. doi: 10.1161/01.res.87.6.460

Papadatos, G. A., Wallerstein, P. M., Head, C. E., Ratcliff, R., Brady, P. A., Benndorf, K., et al. (2002). Slowed conduction and ventricular tachycardia after targeted disruption of the cardiac sodium channel gene Scn5a. Proc. Natl. Acad. Sci. U.S.A. 99, 6210-6215. doi: 10.1073/pnas.082121299

Parra, E., Cruz, D., García, G., Zazueta, C., Correa, F., García, N., et al. (2005). Myocardial protective effect of octylguanidine against the damage induced by ischemia reperfusion in rat heart. Mol. Cell Biochem. 269, 19-26. doi: 10.1007/ s11010-005-2989-0

Paul-Pletzer, K., Yamamoto, T., Bhat, M. B., Ma, J., Ikemoto, N., Jimenez, L. S., et al. (2002). Identification of a dantrolene-binding sequence on the skeletal muscle ryanodine receptor. J. Biol. Chem. 277, 34918-34923. doi: 10.1074/jbc. $\mathrm{m} 205487200$

Pavón, N., Aranda, A., García, N., Hernández-Esquivel, L., and Chávez, E. (2009). In hyperthyroid rats octylguanidine protects the heart from reperfusion damage. Endocrine 35, 158-165. doi: 10.1007/s12020-008-9144-0

Penttinen, K., Swan, H., Vanninen, S., Paavola, J., Lahtinen, A. M., Kontula, K., et al. (2015). Antiarrhythmic effects of dantrolene in patients with catecholaminergic polymorphic ventricular tachycardia and replication of the responses using iPSC models. PLoS One 10:e0125366. doi: 10.1371/journal.pone.0125366

Purohit, A., Rokita, A. G., Guan, X., Chen, B., Koval, O. M., Voigt, N., et al. (2013). Oxidized $\mathrm{Ca}(2+) /$ calmodulin-dependent protein kinase II triggers atrial fibrillation. Circulation 128, 1748-1757. doi: 10.1161/circulationaha.113. 003313

Qin, F., Siwik, D. A., Lancel, S., Zhang, J., Kuster, G. M., Luptak, I., et al. (2013). Hydrogen peroxide-mediated SERCA cysteine 674 oxidation contributes to impaired cardiac myocyte relaxation in senescent mouse heart. J. Am. Heart Assoc. 2:e000184.

Qin, Y., Li, A., Liu, B., Jiang, W., Gao, M., Tian, X., et al. (2020). Mitochondrial fusion mediated by fusion promotion and fission inhibition directs adult mouse heart function toward a different direction. FASEB J. 34, 663-675. doi: 10.1096/ fj.201901671r

Qiu, Y., Bernier, M., and Hearse, D. J. (1990). The influence of N-acetylcysteine on cardiac function and rhythm disorders during ischemia and reperfusion. Cardioscience 1, 65-74.

Ramesh, V., Sharma, V. K., Sheu, S. S., and Franzini-Armstrong, C. (1998). Structural proximity of mitochondria to calcium release units in rat ventricular myocardium may suggest a role in Ca2+ sequestration. Ann. N.Y. Acad. Sci. 853, 341-344. doi: 10.1111/j.1749-6632.1998.tb08295.X

Robinson, P., Liu, X., Sparrow, A., Patel, S., Zhang, Y. H., Casadei, B., et al. (2018). Hypertrophic cardiomyopathy mutations increase myofilament $\mathrm{Ca}(2+)$ buffering, alter intracellular $\mathrm{Ca}(2+)$ handling, and stimulate $\mathrm{Ca}(2+)$-dependent signaling. J. Biol. Chem. 293, 10487-10499. doi: 10.1074/jbc.ra118.002081

Rocca, C., Boukhzar, L., Granieri, M. C., Alsharif, I., Mazza, R., Lefranc, B., et al. (2018). A selenoprotein T-derived peptide protects the heart against ischaemia/reperfusion injury through inhibition of apoptosis and oxidative stress. Acta Physiol. 223:e13067. doi: 10.1111/apha.13067

Rodrigo, R., Korantzopoulos, P., Cereceda, M., Asenjo, R., Zamorano, J., Villalabeitia, E., et al. (2013). A randomized controlled trial to prevent postoperative atrial fibrillation by antioxidant reinforcement. J. Am. Coll. Cardiol. 62, 1457-1465. doi: 10.1016/j.jacc.2013.07.014

Rodríguez-Reyes, H., Muñoz Gutiérrez, M., Márquez, M. F., Pozas Garza, G., Asensio Lafuente, E., Ortíz Galván, F., et al. (2015). Muerte súbita cardiaca. Estratificación de riesgo, prevención y tratamiento. Archivos Cardiol. México 85, 329-336. doi: 10.1016/j.acmx.2015.06.002

Roussel, J., Thireau, J., Brenner, C., Saint, N., Scheuermann, V., Lacampagne, A., et al. (2015). Palmitoyl-carnitine increases RyR2 oxidation and sarcoplasmic reticulum $\mathrm{Ca}^{2+}$ leak in cardiomyocytes: role of adenine nucleotide translocase. Biochim. Biophys. Acta 1852, 749-758. doi: 10.1016/j.bbadis.2015.01.011

Rutledge, C., and Dudley, S. (2013). Mitochondria and arrhythmias. Expert Rev. Cardiovasc. Ther. 11, 799-801. doi: 10.1586/14779072.2013.811969

Sag, C. M., Wadsack, D. P., Khabbazzadeh, S., Abesser, M., Grefe, C., Neumann, K., et al. (2009). Calcium/calmodulin-dependent protein kinase II contributes to cardiac arrhythmogenesis in heart failure. Circ. Heart Fail. 2, 664-675. doi: 10.1161/circheartfailure.109.865279

Sano, T., Sugiyama, S., Taki, K., Hanaki, Y., Shimada, Y., and Ozawa, T. (1990). Effects of antiarrhythmic agents classified as class III group on ischaemiainduced myocardial damage in canine hearts. Br. J. Pharmacol. 99, 577-581. doi: 10.1111/j.1476-5381.1990.tb12971.x

Santana, L. F., Kranias, E. G., and Lederer, W. J. (1997). Calcium sparks and excitation-contraction coupling in phospholamban-deficient mouse ventricular myocytes. J. Physiol. 503(Pt 1), 21-29. doi: 10.1111/j.1469-7793.1997.021bi.x

Santulli, G., Xie, W., Reiken, S. R., and Marks, A. R. (2015). Mitochondrial calcium overload is a key determinant in heart failure. Proc. Natl. Acad. Sci. U.S.A. 112, 11389-11394. doi: 10.1073/pnas.1513047112

Sardu, C., Santulli, G., Guerra, G., Trotta, M. C., Santamaria, M., Sacra, C., et al. (2020). Modulation of SERCA in patients with persistent atrial fibrillation treated by epicardial thoracoscopic ablation: the CAMAF study. J. Clin. Med. 9:544. doi: 10.3390/jcm9020544

Sasaki, K., Makiyama, T., Yoshida, Y., Wuriyanghai, Y., Kamakura, T., Nishiuchi, S., et al. (2016). Patient-specific human induced pluripotent stem cell model assessed with electrical pacing validates $\mathrm{S} 107$ as a potential therapeutic agent for Catecholaminergic polymorphic ventricular tachycardia. PLoS One 11:e0164795. doi: 10.1371/journal.pone.0164795

Schaper, J., Meiser, E., and Stammler, G. (1985). Ultrastructural morphometric analysis of myocardium from dogs, rats, hamsters, mice, and from human hearts. Circ. Res. 56, 377-391. doi: 10.1161/01.res.56.3.377

Schmidt, A. G., Gerst, M., Zhai, J., Carr, A. N., Pater, L., Kranias, E. G., et al. (2002). Evaluation of left ventricular diastolic function from spectral and color M-mode doppler in genetically altered mice. J. Am. Soc. Echocardiogr. 15(10 Pt 1), 1065-1073. doi: 10.1067/mje.2002.121863

Schreiner, K. D., Kelemen, K., Zehelein, J., Becker, R., Senges, J. C., Bauer, A., et al. (2004). Biventricular hypertrophy in dogs with chronic AV block: effects of cyclosporin A on morphology and electrophysiology. Am. J. Physiol. Heart Circ. Physiol. 287, H2891-H2898. doi: 10.1152/ajpheart.01051. 2003

Schweitzer, M. K., Wilting, F., Sedej, S., Dreizehnter, L., Dupper, N. J., Tian, Q., et al. (2017). Suppression of arrhythmia by enhancing mitochondrial $\mathrm{Ca}^{2+}$ uptake in Catecholaminergic ventricular tachycardia models. JACC Basic Transl. Sci. 2, 737-747. doi: 10.1016/j.jacbts.2017.06.008

Scriven, D. R., Asghari, P., and Moore, E. D. (2013). Microarchitecture of the dyad. Cardiovasc. Res. 98, 169-176. doi: 10.1093/cvr/cvt025

Shaikh, G., Zhang, J., Perez-Aso, M., Mediero, A., and Cronstein, B. (2016). Adenosine $\mathrm{A}(2 \mathrm{~A})$ receptor promotes collagen type III synthesis via $\beta$-catenin activation in human dermal fibroblasts. Br. J. Pharmacol. 173, 3279-3291. doi: 10.1111/bph.13615

Shan, J., Xie, W., Betzenhauser, M., Reiken, S., Chen, B. X., Wronska, A., et al. (2012). Calcium leak through ryanodine receptors leads to atrial fibrillation in 3 mouse models of catecholaminergic polymorphic ventricular tachycardia. Circ. Res. 111, 708-717. doi: 10.1161/circresaha.112.273342

Sharma, V., Abraham, T., So, A., Allard, M. F., and McNeill, J. H. (2010). Functional effects of protein kinases and peroxynitrite on cardiac carnitine palmitoyltransferase-1 in isolated mitochondria. Mol. Cell Biochem. 337, $223-$ 237. doi: 10.1007/s11010-009-0303-2

Sheu, S. S., and Lederer, W. J. (1985). Lidocaine's negative inotropic and antiarrhythmic actions. Dependence on shortening of action potential duration and reduction of intracellular sodium activity. Circ. Res. 57, 578-590. doi: 10.1161/01.res.57.4.578

Shimizu, H., Schredelseker, J., Huang, J., Lu, K., Naghdi, S., Lu, F., et al. (2015). Mitochondrial $\mathrm{Ca}^{2+}$ uptake by the voltage-dependent anion channel 2 regulates cardiac rhythmicity. eLife 4:e04801. doi: 10.7554/eLife.04801

Smeets, J. L., Allessie, M. A., Lammers, W. J., Bonke, F. I., and Hollen, J. (1986). The wavelength of the cardiac impulse and reentrant arrhythmias in isolated rabbit atrium. The role of heart rate, autonomic transmitters, temperature, and potassium. Circ. Res. 58, 96-108. doi: 10.1161/01.res.58.1.96 
Smith, R. M., Visweswaran, R., Talkachova, I., Wothe, J. K., and Tolkacheva, E. G. (2013). Uncoupling the mitochondria facilitates alternans formation in the isolated rabbit heart. Am. J. Physiol. Heart Circ. Physiol. 305, H9-H18.

Sochman, J., Kolc, J., Vrána, M., and Fabián, J. (1990). Cardioprotective effects of $\mathrm{N}$-acetylcysteine: the reduction in the extent of infarction and occurrence of reperfusion arrhythmias in the dog. Int. J. Cardiol. 28, 191-196. doi: 10.1016/ 0167-5273(90)90060-i

Soloviev, A., Stefanov, A., Parshikov, A., Khromov, A., Moibenko, A., Kvotchina, L., et al. (2002). Arrhythmogenic peroxynitrite-induced alterations in mammalian heart contractility and its prevention with quercetin-filled liposomes. Cardiovasc. Toxicol. 2, 129-139. doi: 10.1385/ct:2:2:129

Sood, S., Chelu, M. G., van Oort, R. J., Skapura, D., Santonastasi, M., Dobrev, D., et al. (2008). Intracellular calcium leak due to FKBP12.6 deficiency in mice facilitates the inducibility of atrial fibrillation. Heart Rhythm. 5, 1047-1054. doi: 10.1016/j.hrthm.2008.03.030

Sossalla S., Fluschnik, N., Schotola, H., Ort, K. R., Neef, S., Schulte, T., et al. (2010). Inhibition of elevated $\mathrm{Ca}^{2+} /$ calmodulin-dependent protein kinase II improves contractility in human failing myocardium. Circ. Res. 107:1150-1161. doi: 10.1161/CIRCRESAHA.110.220418

Srinivasan, N. T., and Schilling, R. J. (2018). Sudden cardiac death and arrhythmias. Arrhythm. Electrophysiol. Rev. 7, 111-117.

Stanger, O., Aigner, I., Schimetta, W., and Wonisch, W. (2014). Antioxidant supplementation attenuates oxidative stress in patients undergoing coronary artery bypass graft surgery. Tohoku J. Exp. Med. 232, 145-154. doi: 10.1620/ tjem.232.145

Stoica, R., De Vos, K. J., Paillusson, S., Mueller, S., Sancho, R. M., Lau, K. F., et al. (2014). ER-mitochondria associations are regulated by the VAPB-PTPIP51 interaction and are disrupted by ALS/FTD-associated TDP-43. Nat. Commun. 5:3996.

Sugiyama, A., Aye, N. N., Sawada, N., and Hashimoto, K. (1999). Cariporide, a highly selective $\mathrm{Na}^{+} / \mathrm{H}^{+}$exchange inhibitor, suppresses the reperfusioninduced lethal arrhythmias and "overshoot" phenomenon of creatine phosphate in situ rat heart. J. Cardiovasc. Pharmacol. 33, 116-121. doi: 10.1097/00005344199901000-00017

Sugiyama, S., Hattori, M., Miyazaki, Y., Nagai, S., and Ozawa, T. (1985). The effect of verapamil on reperfusion arrhythmia in canine heart. Jpn. Circ. J. 49, 1235-1242. doi: 10.1253/jcj.49.1235

Sventzouri, S., Nanas, I., Vakrou, S., Kapelios, C., Sousonis, V., Sfakianaki, T., et al. (2018). Pharmacologic inhibition of the mitochondrial $\mathrm{Na}^{+} / \mathrm{Ca}^{2+}$ exchanger protects against ventricular arrhythmias in a porcine model of ischemiareperfusion. Hellen. J. Cardiol. 59, 217-222. doi: 10.1016/j.hjc.2017.12.009

Szabadkai, G., Bianchi, K., Várnai, P., De Stefani, D., Wieckowski, M. R., Cavagna, D., et al. (2006). Chaperone-mediated coupling of endoplasmic reticulum and mitochondrial Ca2+ channels. J. Cell Biol. 175, 901-911. doi: 10.1083/jcb. 200608073

Takimoto, E., and Kass, D. A. (2007). Role of oxidative stress in cardiac hypertrophy and remodeling. Hypertension 49, 241-248. doi: 10.1161/01.hyp.0000254415. 31362.a7

Terentyev, D., Györke, I., Belevych, A. E., Terentyeva, R., Sridhar, A., Nishijima, Y., et al. (2008). Redox modification of ryanodine receptors contributes to sarcoplasmic reticulum Ca2+ leak in chronic heart failure. Circ. Res. 103, 1466-1472. doi: 10.1161/circresaha.108.184457

Testai, L., Marino, A., Piano, I., Brancaleone, V., Tomita, K., Di Cesare Mannelli, L., et al. (2016). The novel $\mathrm{H}_{2} \mathrm{~S}$-donor 4-carboxyphenyl isothiocyanate promotes cardioprotective effects against ischemia/reperfusion injury through activation of mitoK $\mathrm{ATP}_{\mathrm{ATP}}$ channels and reduction of oxidative stress. Pharmacol. Res. 113(Pt A), 290-299. doi: 10.1016/j.phrs.2016.09.006

Toda, T., Kadono, T., Hoshiai, M., Eguchi, Y., Nakazawa, S., Nakazawa, H., et al. (2007). $\mathrm{Na}^{+} / \mathrm{H}^{+}$exchanger inhibitor cariporide attenuates the mitochondrial $\mathrm{Ca}^{2+}$ overload and PTP opening. Am. J. Physiol. Heart Circ. Physiol. 293, H3517-H3523. doi: 10.1152/ajpheart.00483.2006

Tränkmann, P., Thiele, R., Winnefeld, K., and Seliger, K. (1999). Effect of administration of selenium and vitamin $\mathrm{E}$ on heart failure and ventricular arrhythmias in patients with acute myocardial infarct. Med. Klin. 94(Suppl. 3), 78-80.

Tsai, M. S., Huang, C. H., Tsai, C. Y., Chen, H. W., Lee, H. C., Cheng, H. J., et al. (2011). Ascorbic acid mitigates the myocardial injury after cardiac arrest and electrical shock. Intens. Care Med. 37, 2033-2040. doi: 10.1007/s00134-0112362-6

Tsutsumi, Y., Oshita, S., Kawano, T., Kitahata, H., Tomiyama, Y., Kuroda, Y., et al. (2001). Lidocaine and mexiletine inhibit mitochondrial oxidation in rat ventricular myocytes. Anesthesiology 95, 766-770. doi: 10.1097/00000542200109000-00032

Turner, D. M., and Walker, J. B. (1985). Relative abilities of phosphagens with different thermodynamic or kinetic properties to help sustain ATP and total adenylate pools in heart during ischemia. Arch. Biochem. Biophys. 238, 642-651. doi: 10.1016/0003-9861(85)90210-3

Ugdyzhekova, D. S., Afanas'ev, S. A., Lukavskaia, I. A., and Popov, S. V. (2005). Cardiac effects of the class III antiarrhythmic drugs amiodarone and nibentan. Fiziol. Cheloveka 31, 113-118.

Ullrich, M., Aßmus, B., Augustin, A. M., Häbich, H., Abeßer, M., Martin Machado, J., et al. (2019). SPRED2 deficiency elicits cardiac arrhythmias and premature death via impaired autophagy. J. Mol. Cell Cardiol. 129, 13-26. doi: 10.1016/j. yjmcc.2019.01.023

Vásquez-Trincado, C., García-Carvajal, I., Pennanen, C., Parra, V., Hill, J. A., Rothermel, B. A., et al. (2016). Mitochondrial dynamics, mitophagy and cardiovascular disease. J. Physiol. 594, 509-525. doi: 10.1113/jp271301

Vazquez Ruiz de Castroviejo, E., Munoz Bellido, J., Lozano Cabezas, C., Ramirez Moreno, A., Guzman Herrera, M., Tarabini Castellani, A., et al. (2005). Analysis of the frequency of cardiac arrhythmias and conduction disturbances from a health-care perspective. Rev. Esp. Cardiol. 58, 657-665. doi: 10.1016/s18855857(06)60252-1

Vázquez-Garza, E., Bernal-Ramírez, J., Jerjes-Sánchez, C., Lozano, O., AcuñaMorín, E., Vanoye-Tamez, M., et al. (2020). Resveratrol prevents right ventricle remodeling and dysfunction in monocrotaline-induced pulmonary arterial hypertension with a limited improvement in the lung vasculature. Oxid. Med. Cell Longev. 2020:1841527.

Verheule, S., Sato, T., Everett, T. T., Engle, S. K., Otten, D., Rubart-von der Lohe, M., et al. (2004). Increased vulnerability to atrial fibrillation in transgenic mice with selective atrial fibrosis caused by overexpression of TGF-beta1. Circ. Res. 94, 1458-1465. doi: 10.1161/01.res.0000129579.59664.9d

Violi, F., Pastori, D., Pignatelli, P., and Loffredo, L. (2014). Antioxidants for prevention of atrial fibrillation: a potentially useful future therapeutic approach? A review of the literature and meta-analysis. Europace 16, 1107-1116. doi: 10.1093/europace/euu040

Vranka, I., Penz, P., and Dukát, A. (2007). Atrial conduction delay and its association with left atrial dimension, left atrial pressure and left ventricular diastolic dysfunction in patients at risk of atrial fibrillation. Exp. Clin. Cardiol. 12, 197-201.

Wai, T., García-Prieto, J., Baker, M. J., Merkwirth, C., Benit, P., Rustin, P., et al. (2015). Imbalanced OPA1 processing and mitochondrial fragmentation cause heart failure in mice. Science 350:aad0116. doi: 10.1126/science.aad0116

Wang, C., Hu, S. M., Xie, H., Qiao, S. G., Liu, H., and Liu, C. F. (2015). Role of mitochondrial ATP-sensitive potassium channel-mediated PKC- $\varepsilon$ in delayed protection against myocardial ischemia/reperfusion injury in isolated hearts of sevoflurane-preconditioned rats. Braz. J. Med. Biol. Res. 48, 528-536. doi: 10.1590/1414-431x20143876

Wang, S., Radhakrishnan, J., Ayoub, I. M., Kolarova, J. D., Taglieri, D. M., and Gazmuri, R. J. (2007). Limiting sarcolemmal Na+ entry during resuscitation from ventricular fibrillation prevents excess mitochondrial $\mathrm{Ca} 2+$ accumulation and attenuates myocardial injury. J. Appl. Physiol. 103, 55-65. doi: 10.1152/ japplphysiol.01167.2006

Wang, Y. G., Liu, C. Z., Li, Y. Z., Peng, Y., and Yan, S. (2020). Cotreatments with Dex and $\mathrm{Na}(2) \mathrm{SeO}(3)$ further improved antioxidant and anti-inflammatory protection of myocardial cells from I/R injury compared to their individual treatments. Free Radic. Res. 54, 76-90. doi: 10.1080/10715762.2019.1707198

Wehrens, X. H., Lehnart, S. E., Huang, F., Vest, J. A., Reiken, S. R., Mohler, P. J., et al. (2003). FKBP12.6 deficiency and defective calcium release channel (ryanodine receptor) function linked to exercise-induced sudden cardiac death. Cell 113, 829-840. doi: 10.1016/s0092-8674(03)00434-3

Wehrens, X. H., Lehnart, S. E., Reiken, S. R., Deng, S. X., Vest, J. A., Cervantes, D., et al. (2004). Protection from cardiac arrhythmia through ryanodine receptorstabilizing protein calstabin2. Science 304, 292-296. doi: 10.1126/science. 1094301 
Wiersma, M., van Marion, D. M. S., Wüst, R. C. I., Houtkooper, R. H., Zhang, D., Groot, N. M. S., et al. (2019). Mitochondrial dysfunction underlies cardiomyocyte remodeling in experimental and clinical Atrial fibrillation. Cells 8:1202. doi: $10.3390 /$ cells 8101202

Wiesmann, T., Freitag, D., Dersch, W., Eschbach, D., Irqsusi, M., Steinfeldt, T., et al. (2017). Dantrolene versus amiodarone for cardiopulmonary resuscitation: a randomized, double-blinded experimental study. Sci. Rep. 7:40875. doi: 10. 1038/srep40875

Wijffels, M. C., Kirchhof, C. J., Dorland, R., and Allessie, M. A. (1995). Atrial fibrillation begets atrial fibrillation. A study in awake chronically instrumented goats. Circulation 92, 1954-1968. doi: 10.1161/01.cir.92.7.1954

Wilson, D. N., Schacht, S. E., Al-Nakkash, L., Babu, J. R., and Broderick, T. L. (2016). Resveratrol prevents pulmonary trunk remodeling but not right ventricular hypertrophy in monocrotaline-induced pulmonary hypertension. Pathophysiology 23, 243-250. doi: 10.1016/j.pathophys.2016.05. 004

Wilting, F., Kopp, R., Gurnev, P. A., Schedel, A., Dupper, N. J., Kwon, O., et al. (2020). The antiarrhythmic compound efsevin directly modulates voltagedependent anion channel 2 by binding to its inner wall and enhancing mitochondrial $\mathrm{Ca}(2+)$ uptake. Br. J. Pharmacol. 177, 2947-2958. doi: 10.1111/ bph. 15022

Wongcharoen, W., Chen, Y. C., Chen, Y. J., Chen, S. Y., Yeh, H. I., Lin, C. I., et al. (2007). Aging increases pulmonary veins arrhythmogenesis and susceptibility to calcium regulation agents. Heart Rhythm. 4, 1338-1349. doi: 10.1016/j. hrthm.2007.06.023

Woodward, B., and Zakaria, M. N. (1985). Effect of some free radical scavengers on reperfusion induced arrhythmias in the isolated rat heart. J. Mol. Cell Cardiol. 17, 485-493. doi: 10.1016/s0022-2828(85)80053-5

Wu, H., Ye, M., Liu, D., Yang, J., Ding, J. W., Zhang, J., et al. (2019). UCP2 protect the heart from myocardial ischemia/reperfusion injury via induction of mitochondrial autophagy. J. Cell Biochem. 120, 15455-15466. doi: 10.1002/ jcb. 28812

Wu, S., Lu, Q., Wang, Q., Ding, Y., Ma, Z., Mao, X., et al. (2017). Binding of FUN14 domain containing 1 With Inositol 1,4,5-trisphosphate receptor in mitochondria-associated endoplasmic reticulum membranes maintains mitochondrial dynamics and function in hearts in vivo. Circulation 136, 22482266. doi: 10.1161/circulationaha.117.030235

Wu, Y., Rasmussen, T. P., Koval, O. M., Joiner, M. L., Hall, D. D., Chen, B., et al. (2015). The mitochondrial uniporter controls fight or flight heart rate increases. Nat. Commun. 6:6081.

Xiao, D., Gu, Z. L., and Qian, Z. N. (1993). Effects of quercetin on platelet and reperfusion-induced arrhythmias in rats. Zhongguo Yao Li Xue Bao 14, 505-508.

Xie, A., Song, Z., Liu, H., Zhou, A., Shi, G., Wang, Q., et al. (2018). Mitochondrial $\mathrm{Ca}^{2+}$ influx contributes to arrhythmic risk in nonischemic cardiomyopathy. J. Am. Heart Assoc. 7:7805. doi: 10.1161/JAHA.117.007805

Xie, J. R., and Yu, L. N. (2007). Cardioprotective effects of cyclosporine A in an in vivo model of myocardial ischemia and reperfusion. Acta Anaesthesiol. Scand. 51, 909-913. doi: 10.1111/j.1399-6576.2007.01342.x

Xie, W., Santulli, G., Reiken, S. R., Yuan, Q., Osborne, B. W., Chen, B. X., et al. (2015). Mitochondrial oxidative stress promotes atrial fibrillation. Sci. Rep. 5:11427.

Xu, D., Murakoshi, N., Igarashi, M., Hirayama, A., Ito, Y., Seo, Y., et al. (2012). PPAR- $\gamma$ activator pioglitazone prevents age-related atrial fibrillation susceptibility by improving antioxidant capacity and reducing apoptosis in a rat model. J. Cardiovasc. Electrophysiol. 23, 209-217. doi: 10.1111/j.1540-8167. 2011.02186.x

Xu, S., Wang, P., Zhang, H., Gong, G., Gutierrez Cortes, N., Zhu, W., et al. (2016). CaMKII induces permeability transition through Drpl phosphorylation during chronic $\beta$-AR stimulation. Nat. Commun. 7:13189.

Yang, K. C., Kyle, J. W., Makielski, J. C., and Dudley, S. C. Jr. (2015). Mechanisms of sudden cardiac death: oxidants and metabolism. Circ. Res. 116, 1937-1955. doi: $10.1161 /$ circresaha.116.304691
Yu, Z., Chen, R., Li, M., Yu, Y., Liang, Y., Han, F., et al. (2018). Mitochondrial calcium uniporter inhibition provides cardioprotection in pressure overloadinduced heart failure through autophagy enhancement. Int. J. Cardiol. 271, 161-168. doi: 10.1016/j.ijcard.2018.05.054

Zhang, J., Corciulo, C., Liu, H., Wilder, T., Ito, M., and Cronstein, B. (2017). Adenosine $\mathrm{A}(2 \mathrm{a})$ receptor blockade diminishes $\mathrm{Wnt} / \beta$-Catenin signaling in a murine model of bleomycin-induced dermal fibrosis. Am. J. Pathol. 187, 1935-1944. doi: 10.1016/j.ajpath.2017.05.005

Zhang, C., Deng, Y., Lei, Y., Zhao, J., Wei, W., and Li, Y. (2017). Effects of selenium on myocardial apoptosis by modifying the activity of mitochondrial STAT3 and regulating potassium channel expression. Exp. Ther. Med. 14, 2201-2205. doi: $10.3892 /$ etm.2017.4716

Zhang, L., Deng, T., Sun, Y., Liu, K., Yang, Y., and Zheng, X. (2008). Role for nitric oxide in permeability of hippocampal neuronal hemichannels during oxygen glucose deprivation. J. Neurosci. Res. 86, 2281-2291. doi: 10.1002/jnr.21675

Zhang, T., Maier, L. S., Dalton, N. D., Miyamoto, S., Ross, J. Jr., Bers, D. M., et al. (2003). The deltaC isoform of CaMKII is activated in cardiac hypertrophy and induces dilated cardiomyopathy and heart failure. Circ. Res. 92, 912-919. doi: 10.1161/01.res.0000069686.31472.c5

Zhang, Y., Shimizu, H., Siu, K. L., Mahajan, A., Chen, J. N., and Cai, H. (2014). NADPH oxidase 4 induces cardiac arrhythmic phenotype in zebrafish. J. Biol. Chem. 289, 23200-23208. doi: 10.1074/jbc.m114.587196

Zhao, W., Frank, K. F., Chu, G., Gerst, M. J., Schmidt, A. G., Ji, Y., et al. (2003). Combined phospholamban ablation and SERCAla overexpression result in a new hyperdynamic cardiac state. Cardiovasc. Res. 57, 71-81. doi: 10.1016/ s0008-6363(02)00609-0

Zhou, L., Solhjoo, S., Millare, B., Plank, G., Abraham, M. R., Cortassa, S., et al. (2014). Effects of regional mitochondrial depolarization on electrical propagation: implications for arrhythmogenesis. Circ. Arrhythm. Electrophysiol. 7, 143-151. doi: 10.1161/circep.113.000600

Zhu, J., Rebecchi, M. J., Tan, M., Glass, P. S., Brink, P. R., and Liu, L. (2010). Age-associated differences in activation of Akt/GSK-3beta signaling pathways and inhibition of mitochondrial permeability transition pore opening in the rat heart. J. Gerontol. A Biol. Sci. Med. Sci. 65, 611-619. doi: 10.1093/gerona/glq035

Zima, A. V., and Blatter, L. A. (2006). Redox regulation of cardiac calcium channels and transporters. Cardiovasc. Res. 71, 310-321. doi: 10.1016/j.cardiores.2006. 02.019

Zissimopoulos, S., Docrat, N., and Lai, F. A. (2007). Redox sensitivity of the ryanodine receptor interaction with FK506-binding protein. J. Biol. Chem. 282, 6976-6983. doi: 10.1074/jbc.m607590200

Zulian, A., Sileikytë, J., Petronilli, V., Bova, S., Dabbeni-Sala, F., Cargnelli, G., et al. (2011). The translocator protein (peripheral benzodiazepine receptor) mediates rat-selective activation of the mitochondrial permeability transition by norbormide. Biochim. Biophys. Acta 1807, 1600-1605. doi: 10.1016/j.bbabio. 2011.08.007

Zulkifly, H., Lip, G. Y. H., and Lane, D. A. (2018). Epidemiology of atrial fibrillation. Int. J. Clin. Pract. 72:e13070.

Zuo, S., Li, L. L., Ruan, Y. F., Jiang, L., Li, X., Li, S. N., et al. (2018). Acute administration of tumour necrosis factor- $\alpha$ induces spontaneous calcium release via the reactive oxygen species pathway in atrial myocytes. Europace 20, 1367-1374. doi: 10.1093/europace/eux271

Conflict of Interest: The authors declare that the research was conducted in the absence of any commercial or financial relationships that could be construed as a potential conflict of interest.

Copyright (- 2021 Salazar-Ramírez, Ramos-Mondragón and García-Rivas. This is an open-access article distributed under the terms of the Creative Commons Attribution License (CC BY). The use, distribution or reproduction in other forums is permitted, provided the original author(s) and the copyright owner(s) are credited and that the original publication in this journal is cited, in accordance with accepted academic practice. No use, distribution or reproduction is permitted which does not comply with these terms. 